\title{
Double beta decay, Majorana neutrinos, and neutrino mass
}

\author{
Frank T. Avignone III* \\ Department of Physics and Astronomy, University of South Carolina, Columbia, South \\ Carolina 29208, USA \\ Steven R. Elliott ${ }^{\dagger}$ \\ Los Alamos National Laboratory, Los Alamos, New Mexico 87545, USA \\ Jonathan Engel ${ }^{\ddagger}$ \\ Department of Physics and Astronomy, University of North Carolina, Chapel Hill, North \\ Carolina 27599-3255, USA
}

(Published 9 April 2008)

\begin{abstract}
The theoretical and experimental issues relevant to neutrinoless double beta decay are reviewed. The impact that a direct observation of this exotic process would have on elementary particle physics, nuclear physics, astrophysics, and cosmology is profound. Now that neutrinos are known to have mass and experiments are becoming more sensitive, even the nonobservation of neutrinoless double beta decay will be useful. If the process is actually observed, we will immediately learn much about the neutrino. The status and discovery potential of proposed experiments are reviewed in this context, with significant emphasis on proposals favored by recent panel reviews. The importance of and challenges in the calculation of nuclear matrix elements that govern the decay are considered in detail. The increasing sensitivity of experiments and improvements in nuclear theory make the future exciting for this field at the interface of nuclear and particle physics.
\end{abstract}

DOI: $10.1103 / \operatorname{RevModPhys.80.481}$

PACS number(s): 11.30.Fs, 14.60.Pq, 23.40.-s

\section{CONTENTS}

I. Introduction

A. The early history

481

B. Overview of theory and recent experimental developments

C. The claimed observation

II. Majorana Neutrinos

III. Rate of Double Beta Decay

IV. Double Beta Decay and New Physics

A. Neutrino mass

B. The Majorana phases

C. Sterile neutrinos

D. Alternative mechanisms

V. Calculating Nuclear Matrix Elements
A. Nuclear-structure theory
B. QRPA
C. Shell model
D. Prospects for the future

VI. Experimental Aspects
A. Background and experimental design
B. Facility requirements
C. Measurements to constrain nuclear matrix elements

VII. Future Program
A. Previous experiments
B. Overview of the future program

\footnotetext{
*avignone@sc.edu

†elliotts@lanl.gov

†engelj@physics.unc.edu
}

1. The number of required experiments and their precision $\quad 500$

2. Kinematic distributions $\quad 500$

C. The experiments 501

1. CUORE (Cryogenic Underground Observatory for Rare Events) 501

2. EXO (Enriched Xenon Observatory) 503

3. Next generation ${ }^{76} \mathrm{Ge}$ double beta decay experiments: MAJORANA and GERDA

4. COBRA (Cadmium Telluride 0-neutrino Beta Decay Research Apparatus)

5. MOON (Molybdenum Observatory of Neutrinos)

6. NEMO-3 and SuperNEMO

7. CANDLES (calcium fluoride for studies of neutrinos and dark matters by low energy spectrometer)

8. Other proposals 511

VIII. Conclusions

Note added in proof

Acknowledgments

References

\section{INTRODUCTION}

Neutrinoless double beta decay $[\beta \beta(0 \nu)]$ is a very slow lepton-number-violating nuclear transition that occurs if neutrinos have mass (which they do) and are their own antiparticles. An initial nucleus $(Z, A)$ with proton number $Z$ and total nucleon number $A$ decays to $(Z+2, A)$, emitting two electrons in the process. A related transition, called two-neutrino double beta decay $[\beta \beta(2 \nu)]$, re- 
sults in the emission of two electron antineutrinos in addition to the electrons, and occurs whether or not neutrinos are their own antiparticles. $\beta \beta(2 \nu)$ has in fact been observed in a number of experiments. With the exception of one unconfirmed observation, on the other hand, $\beta \beta(0 \nu)$ has never been seen, and searches for it are ongoing in a number of laboratories around the world. Other even slower and more exotic processes, including double-positron decay, double-electron capture, and neutrinoless decay with the emission of a hypothetical particle called the Majoron $[\beta \beta(0 \nu, \chi)]$, have likewise never been observed.

The development of effective-field theory and grandunification schemes in the late 1970s and early 1980s led to the expectation that neutrinos are identical with their antiparticles and have nonzero mass, and engendered renewed interest in $\beta \beta(0 \nu)$ experiments. More recently, neutrino-oscillation experiments have yielded compelling evidence that the three observed flavors of neutrinos are not mass eigenstates but rather linear combinations of those eigenstates (at least two of which have nonzero mass eigenvalues). These experiments also allow the electron neutrino to mix significantly with the heaviest of the mass eigenstates. If it does, the effective neutrino mass will be large enough that $\beta \beta(0 \nu)$ may well be observed in experiments currently under construction or development. An observation would establish that neutrinos are "Majorana" particles $(\nu \equiv \bar{\nu}$, roughly speaking), and a measurement of the decay rate, when combined with neutrino-oscillation data, would yield insight into all three neutrino-mass eigenstates. This paper is motivated by the recent developments in neutrino physics and by the interest among physicists throughout the world in a coherent experimental $\beta \beta(0 \nu)$ program.

\section{A. The early history}

Double beta decay was first considered in a 1935 paper by Maria Goeppert-Mayer (1935). The author, who acknowledged Eugene Wigner for suggesting the problem, derived an expression for the decay rate and estimated a half-life of $\sim 10^{17} \mathrm{yr}$ for a decay with the emission of two electrons and two antineutrinos $(\bar{\nu})$, carrying about $10 \mathrm{MeV}$ of energy. Two years later Ettore Majorana formulated a theory of neutrinos in which there was no distinction between $\nu$ and $\bar{\nu}$ (Majorana, 1937), and suggested an experimental test of his hypothesis with a reaction similar to $\bar{\nu}_{e}+{ }^{37} \mathrm{Cl} \rightarrow{ }^{37} \mathrm{Ar}+e^{-}$, which was later searched for (and not found) by Raymond Davis (Davis, 1955). It was Giulio Racah, however, who first suggested testing Majorana's theory with $\beta \beta(0 \nu)$ (Racah, 1937). In 1939 Furry calculated approximate rates for $\beta \beta(0 \nu)$ (Furry, 1939), and in 1952 Primakoff (Primakoff, 1952) calculated the electron-electron angular correlations and electron energy spectra for both $\beta \beta(2 \nu)$ and $\beta \beta(0 \nu)$, producing a useful tool for distinguishing between the two processes. These early contributions set the stage for many years of experimental and theoretical activity.

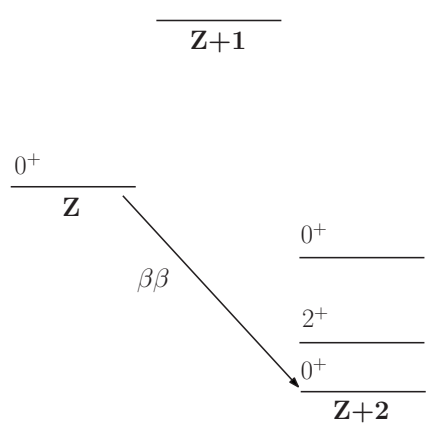

FIG. 1. A generic level diagram for $\beta \beta$.

The review by Haxton and Stephenson (1984) contains a chronology of experiments from 1948 through 1983. There were some early claims of observation. Fireman (1949) reported observing the $\beta \beta$ of ${ }^{124} \mathrm{Sn}$ in a laboratory experiment, but retracted the claim later (Fireman, 1952). The first geochemical observation of $\beta \beta$, with an estimated half-life of $T_{1 / 2}^{\beta \beta}\left({ }^{130} \mathrm{Te}\right)=1.4 \times 10^{21} \mathrm{yr}$, was reported in 1950 (Ingram and Reynolds, 1950). The first actual laboratory observation of $\beta \beta(2 \nu)$ was not made until 1987 (Elliott et al., 1987). Here we concentrate on experiments developed since the late 1980s, referencing earlier work where appropriate. The early developments have been covered well in other reviews, for example, Primakoff and Rosen (1981); Haxton and Stephenson (1984); Doi et al. (1985); Avignone and Brodzinski (1988); Tomoda (1991); Moe and Vogel (1994); Faessler and Šimkovic (1998); Suhonen and Civitarese (1998); Elliott and Vogel (2002); Tretyak and Zdesenko (2002); Zdesenko (2002); Elliott and Engel (2004); Avignone et al. (2005); Ejiri (2005).

\section{B. Overview of theory and recent experimental developments}

A typical $\beta \beta$ candidate is an even-even nucleus $(Z, A)$ which pairing forces make more bound than its $(Z$ $+1, A)$ neighbor, but less so than the $(Z+2, A)$ nuclide, as shown in Fig. 1. In Fig. 2 we depict $\beta \beta(2 \nu)$ and neutrino-exchange-driven $\beta \beta(0 \nu)$. The rate of $\beta \beta(2 \nu)$, which has been measured in ten isotopes (see Table II), can be written as

$$
\left(T_{1 / 2}^{2 \nu}\right)^{-1}=G_{2 \nu}\left(Q_{\beta \beta}, Z\right)\left|M_{2 \nu}\right|^{2}
$$

where $G_{2 \nu}\left(Q_{\beta \beta}, Z\right)$ is the four-particle phase-space factor, and $M_{2 v}$ is a nuclear matrix element for this secondorder process. This decay conserves lepton number, does not discriminate between Majorana and Dirac neutrinos, and does not depend significantly on the masses of the neutrinos. The rate of $\beta \beta(0 \nu)$, if driven by the exchange of light Majorana neutrinos, is approximately

$$
\left(T_{1 / 2}^{0 \nu}\right)^{-1}=G_{0 \nu}\left(Q_{\beta \beta}, Z\right)\left|M_{0 \nu}\right|^{2}\left\langle m_{\beta \beta}\right\rangle^{2},
$$

where $G_{0 \nu}\left(Q_{\beta \beta}, Z\right)$ is the phase-space factor for the emission of the two electrons, $M_{0 \nu}$ is another nuclear matrix element, and $\left\langle m_{\beta \beta}\right\rangle$ is the effective Majorana mass of the electron neutrino: 

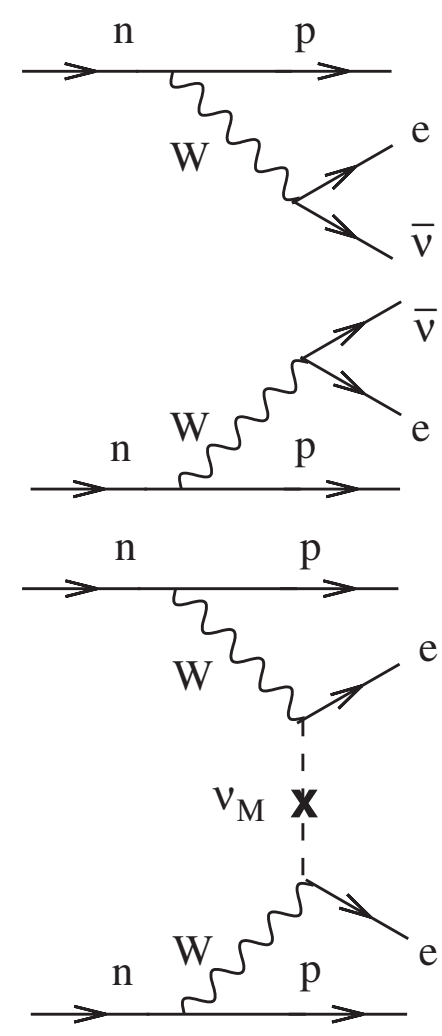

FIG. 2. Feynman diagrams for $\beta \beta(2 \nu)$ (top) and $\beta \beta(0 \nu)$ (bottom).

$$
\left\langle m_{\beta \beta}\right\rangle \equiv\left|\sum_{k} m_{k} U_{e k}^{2}\right| .
$$

Here the $m_{k}$ 's are the masses of the three light neutrinos and $U$ is the matrix that transforms states with welldefined mass into states with well-defined flavor (e.g., electron, mu, tau). Equation (2) gives the $\beta \beta(0 \nu)$ rate if the exchange of light Majorana neutrinos with lefthanded interactions is responsible. Other mechanisms are possible (see Secs. III and IV.D), but they require the existence of new particles and/or interactions in addition to requiring that neutrinos be Majorana particles. Lightneutrino exchange is therefore, in some sense, the "minima" mechanism and the most commonly considered.

That neutrinos mix and have mass is now accepted wisdom. Oscillation experiments constrain $U$ fairly well-Table I summarizes our current knowledge-but they determine only the differences between the squares of the masses $m_{k}$ (e.g., $m_{2}^{2}-m_{1}^{2}$ ) rather than the masses themselves. It will turn out that $\beta \beta(0 \nu)$ is among the best ways of getting at the masses (along with cosmology and $\beta$-decay measurements), and the only practical way to establish that neutrinos are Majorana particles.

To extract the effective mass from a measurement, it is customary to define a nuclear structure factor $F_{N}$ $\equiv G_{0 \nu}\left(Q_{\beta \beta}, Z\right)\left|M_{0 \nu}\right|^{2} m_{e}^{2}$, where $m_{e}$ is the electron mass. (The quantity $F_{N}$ is sometimes written as $C_{m m}$.) The effective mass $\left\langle m_{\beta \beta}\right\rangle$ can be written in terms of the calculated $F_{N}$ and the measured half-life as

$$
\left\langle m_{\beta \beta}\right\rangle=m_{e}\left[F_{N} T_{1 / 2}^{0 \nu}\right]^{-1 / 2} .
$$

The range of mixing matrix values given in Table I, combined with calculated values for $F_{N}$, allow us to estimate the half-life a given experiment must be able to measure in order to be sensitive to a particular value of $\left\langle m_{\beta \beta}\right\rangle$. Published values of $F_{N}$ are typically between $10^{-13}$ and $10^{-14} \mathrm{yr}^{-1}$. To reach a sensitivity of $\left\langle m_{\beta \beta}\right\rangle \approx 0.1 \mathrm{eV}$ therefore an experiment must be able to observe a half-life of $10^{26}-10^{27} \mathrm{yr}$. As we discuss later, at this level of sensitivity an experiment can draw important conclusions whether or not the decay is observed.

The most sensitive limits thus far are from the Heidelberg-Moscow experiment: $T_{1 / 2}^{0 \nu}\left({ }^{76} \mathrm{Ge}\right) \geqslant 1.9 \times 10^{25}$ yr (Baudis et al., 1999), the IGEX experiment: $T_{1 / 2}^{0 \nu}\left({ }^{76} \mathrm{Ge}\right) \geqslant 1.6 \times 10^{25} \mathrm{yr}$ (Aalseth et al., 2002a, 2004), and the CUORICINO experiment: $T_{1 / 2}^{0 \nu}\left({ }^{130} \mathrm{Te}\right) \geqslant 3.0$ $\times 10^{24}$ yr (Arnaboldi et al., 2005, 2007). These experiments contained $5-10 \mathrm{~kg}$ of the parent isotope and ran for several years. Hence increasing the half-life sensitiv-

TABLE I. Neutrino mixing parameters as summarized by the Particle Data Book [Yao et al. (2006)] based on the individual experimental reference reporting. The limit on $\left\langle m_{\beta}\right\rangle$ and $\Sigma$ are based on the references given. The $\left\langle m_{\beta \beta}\right\rangle$ limit comes from the Ge experiments. The parameter values would be slightly different if determined by a global fit to all oscillation data (Fogli et al., 2006).

\begin{tabular}{lccc}
\hline \hline Parameter & Value & Confidence level & Reference \\
\hline $\sin ^{2}\left(2 \theta_{12}\right)$ & $0.86_{-0.04}^{+0.03}$ & $68 \%$ & Aharmin et al. $(2005)$ \\
$\sin ^{2}\left(2 \theta_{23}\right)$ & $>0.92$ & $90 \%$ & Ashie et al. $(2005)$ \\
$\sin ^{2}\left(2 \theta_{13}\right)$ & $<0.19$ & $90 \%$ & Apollonio et al. $(1999)$ \\
$\Delta m_{21}^{2}$ & $8.0_{-0.3}^{+0.4} \times 10^{-5} \mathrm{eV}^{2}$ & $68 \%$ & Aharmin et al. $(2005)$ \\
$\left|\Delta m_{32}^{2}\right|$ & $2.4_{-0.5}^{+0.6} \times 10^{-3} \mathrm{eV}^{2}$ & $90 \%$ & Ashie et al. (2004) \\
$\left\langle m_{\beta}\right\rangle$ & $\left.<2 \mathrm{eV}_{\beta \beta}\right\rangle$ & $95 \%$ & Lobashev et al. (1999); Kraus et al. (2005) \\
$\left\langle m_{\beta}\right.$ & $<0.7 \mathrm{eV}^{\mathrm{a}}$ & $90 \%$ & Klapdor-Kleingrothaus et al. (2001a); Aalseth \\
$\Sigma$ & $<2 \mathrm{eV}$ & $95 \%$ & et al. (2002a) \\
\hline \hline
\end{tabular}

${ }^{\mathrm{a}}$ Using the matrix element of Rodin et al. (2006). 
ity by a factor of about 100 , the goal of the next generation of experiments, will require hundreds of $\mathrm{kg}$ of parent isotope and a significant decrease in background beyond the present state of the art [roughly 0.1 count/(keV kg yr)].

It is straightforward to derive an approximate analytical expression for the half-life to which an experiment with a given level of background is sensitive (Avignone et al., 2005):

$$
T_{1 / 2}^{0 \nu}\left(n_{\sigma}\right)=\frac{4.16 \times 10^{26} \mathrm{yr}}{n_{\sigma}}\left(\frac{\varepsilon a}{W}\right) \sqrt{\frac{M t}{b \Delta(E)}} .
$$

Here $n_{\sigma}$ is the number of standard deviations corresponding to a given confidence level (CL) - a CL of $99.73 \%$ corresponds to $n_{\sigma}=3$ - the quantity $\varepsilon$ is the event-detection and identification efficiency, $a$ is the isotopic abundance, $W$ is the molecular weight of the source material, and $M$ is the total mass of the source. The instrumental spectral width $\Delta(E)$, defining the signal region, is related to the energy resolution at the energy of the expected $\beta \beta(0 \nu)$ peak, and $b$ is the specific background rate in counts/(keV kg yr), where the mass is that of the source, as opposed to the isotope. Equation (5) is valid only if the background level is large enough so that the uncertainty is proportional to $\sqrt{b \Delta(E)}$. For a $200-\mathrm{kg}$ ${ }^{76} \mathrm{Ge}$ experiment with a background rate of 0.01 count $/(\mathrm{keV} \mathrm{kg} \mathrm{yr})$ and an energy resolution of $3.5 \mathrm{keV}$, running for $5 \mathrm{yr}$, the values for these parameters are $M t=10^{3} \mathrm{~kg} \mathrm{yr}, \varepsilon=0.95, a=0.86, W=76$, and $\Delta(E)=3.5 \mathrm{keV}$. This results in a $4 \sigma$ half-life sensitivity of $T_{1 / 2}^{0 \nu}\left(4 \sigma,{ }^{76} \mathrm{Ge}\right)=1.9 \times 10^{26} \mathrm{yr}$. The background rate quoted above is conservative for a Ge experiment, only a factor of 6 below that of the Heidelberg-Moscow and IGEX experiments. A background rate of 0.001 count/(keV kg yr) would allow a $4 \sigma$ discovery with $T_{1 / 2}^{0 \nu}=6 \times 10^{26} \mathrm{yr}$. But an experiment with even modestly lower efficiency or poorer resolution must attain much lower background rates to have comparable sensitivity.

These numbers characterize the level future experiments will have to reach to make a significant contribution to the field. Later we discuss a number of proposed experiments and attempt to estimate their sensitivity.

\section{The claimed observation}

In 2001, a subset of the Heidelberg-Moscow Collaboration (Klapdor-Kleingrothaus et al., 2001a, 2001b, 2003; Kleingrothaus and Krivosheina, 2006) claimed to observe evidence for a $\beta \beta(0 \nu)$ peak in the spectrum of their ${ }^{76} \mathrm{Ge}$ experiment at $2039 \mathrm{keV}$. This claim and later papers by the same group elicited a number of critical replies, for example, Harney (2001); Aalseth et al. (2002b); Feruglio et al. (2002); Zdesenko et al. (2002). But whether or not the result is valid, the experiment was the most sensitive to date. The parameter values were $M t=71.7 \mathrm{~kg} \mathrm{yr}, b=0.11 \mathrm{count} /(\mathrm{keV} \mathrm{kg} \mathrm{yr}), \varepsilon=0.95$, $a=0.86, W=76$, and $\Delta(E)=3.27 \mathrm{keV}$. The number of counts under the peak at $2039 \mathrm{keV}$ was $28.75 \pm 6.86$ (Klapdor-Kleingrothaus et al., 2004a). Substitution into Eq. (5) yields $T_{1 / 2}^{0 \nu}\left(4^{\sigma},{ }^{76} \mathrm{Ge}\right)=1.6 \times 10^{25} \mathrm{yr}$, a lifetime comparable to the claimed most probable value, 2.23 $\times 10^{25} \mathrm{yr}$. At least nominally, the experiment had a $4 \sigma$ discovery potential, and cannot be dismissed out of hand. Since this analysis does not account for statistical fluctuations, background structure, or systematic uncertainties, the actual confidence level could be significantly different. But the only certain way to confirm or refute the claim is with additional experimentation, preferably in ${ }^{76} \mathrm{Ge}$.

To this end, the GERDA experiment is under construction in the LNGS (Abt et al., 2004) and the MAJORANA project (Gaitskell et al., 2003) is being developed in the U.S. The CUORICINO experiment in the LNGS (Arnaboldi et al., 2005) uses ${ }^{130} \mathrm{Te}$, and is the most sensitive experiment currently operating, with a lower bound of $T_{1 / 2}^{0 \nu}\left({ }^{130} \mathrm{Te}\right) \geqslant 3 \times 10^{24} \mathrm{yr}$. This limit is at or near the sensitivity needed to test the 2001 claim, but uncertainty in the calculated value of the nuclear matrix element $M_{0 \nu}$ (or, equivalently, $F_{N}$ ) will preclude a definitive statement.

Foiled by the nuclear matrix elements, one can see even in this brief overview how nice it would be to have accurate matrix elements. We address the issue of how well they can be calculated later.

\section{MAJORANA NEUTRINOS}

As we see, $\beta \beta(0 \nu)$ cannot occur unless neutrinos are Majorana particles, i.e., their own antiparticles. We therefore briefly review the nature of neutral fermions. Much of what we say here is covered in a similar fashion but much greater depth in Bilenky and Petcov (1987).

We can project an arbitrary four-spinor $\Psi$ onto states of definite chirality, which are singlets under one of the two $\mathrm{SU}(2)$ algebras that make up the Lorentz algebra $\mathrm{SO}(3,1)$. We define the left- and right-handed projections as $\Psi_{L, R}=\left[\left(1 \mp \gamma_{5}\right) / 2\right] \Psi$. Because of the minus sign in the Minkowski metric and the resulting need to work with $\bar{\Psi} \equiv \Psi^{\dagger} \gamma_{0}$ rather than $\Psi^{\dagger}$ alone, a Lorentz scalar cannot be constructed by contracting two left-handed spinors in the usual way. Instead one must contract a left-handed spinor with a right-handed one. The (scalar) term in the Lagranginan obtained in this way is called the Dirac mass term:

$$
\mathcal{L}_{D}=-m_{D} \bar{\Psi} \Psi=-m_{D}\left(\overline{\Psi_{L}} \Psi_{R}+\overline{\Psi_{R}} \Psi_{L}\right) .
$$

The terms above contract $\Psi_{L}^{*}$ with $\Psi_{R}$ (and vice versa), with $\gamma_{0}$ flipping the chirality so that the contraction can be made.

If charge conservation is not required, one can form a scalar by combining $\Psi$ with itself rather than with $\Psi^{*}$. Such a term cannot exist in the Lagrangian for electrons or quarks because it destroys or creates two particles of the same charge (or destroys a particle while creating an antiparticle of opposite charge), but nothing forbids it in 
the Lagrangian of neutral fermions such as neutrinos. It violates lepton number, a global U(1) symmetry that tracks the difference between particle number and antiparticle number, but there is nothing sacred about global symmetries. Indeed, $\beta \beta(0 \nu)$ can not occur unless leptonnumber symmetry is violated.

To construct a Lorentz scalar from two $\Psi$ 's, we note that the charge conjugate of $\Psi$, defined up to an arbitrary phase as $\Psi^{c} \equiv \gamma^{2} \Psi$, transforms in the correct way; its chirality is opposite that of $\Psi$ itself because $\gamma^{2}$ and $\gamma_{5}$ anticommute. Thus $\left(\Psi_{L}\right)^{c}$ is right handed, and we can construct left- and right-handed Majorana mass terms of the form

$$
\mathcal{L}_{M}=-\frac{m_{L}}{2}\left[\overline{\left(\Psi_{L}\right)^{c}} \Psi_{L}+\text { H.c. }\right]-\frac{m_{R}}{2}\left[\overline{\left(\Psi_{R}\right)^{c}} \Psi_{R}+\text { H.c. }\right] .
$$

Although we have used all four Dirac components in this equation, it is possible that only the two in $\Psi_{L}$ actually exist if there is no Dirac mass term.

Equations (6) and (7) can be generalized to include $N$ flavors. If, following Bilenky and Petcov (1987) and letting $\Psi$ be the neutrino field $\nu$, we define

$$
n_{L} \equiv\left[\begin{array}{c}
\nu_{L} \\
\left(\nu_{R}\right)^{c}
\end{array}\right]
$$

where

$$
\nu_{L} \equiv\left[\begin{array}{c}
\nu_{e L} \\
\nu_{\mu L} \\
\nu_{\tau L} \\
\vdots
\end{array}\right], \quad\left(\nu_{R}\right)^{c} \equiv\left[\begin{array}{c}
\left(\nu_{e R}\right)^{c} \\
\left(\nu_{\mu R}\right)^{c} \\
\left(\nu_{\tau R}\right)^{c} \\
\vdots
\end{array}\right],
$$

then we find for the mass part of the Lagrangian

$$
\mathcal{L}_{D+M}=-\frac{1}{2} \overline{\left(n_{L}\right)^{c}} \mathcal{M} n_{L}+\text { H.c. }, \quad \mathcal{M}=\left(\begin{array}{ll}
\mathcal{M}_{L} & \mathcal{M}_{D}^{T} \\
\mathcal{M}_{D} & \mathcal{M}_{R}
\end{array}\right) .
$$

The (generically complex symmetric) $N \times N$ matrices $\mathcal{M}_{L}$ and $\mathcal{M}_{R}$ are the Majorana mass terms and the $N$ $\times N$ matrix $\mathcal{M}_{D}$ is the Dirac term. Except in special cases, the eigenstates of $\mathcal{L}_{D+M}$ will correspond to Majorana particles. The Lagrangian is invariant under $C P T$, a transformation that takes a particle into an antiparticle with spin direction reversed. Since the eigenstates will in general consist of $2 \mathrm{~N}$ nondegenerate spinors, the components in each spinor must transform into each under $C P T$, rather than into an orthogonal spinor, so that the neutrinos will be their own antiparticles. To see this more precisely, we note that $\mathcal{M}$, if nondegenerate, can be rewritten in the form $\mathcal{M}=\left(\mathcal{U}^{\dagger}\right)^{T} \hat{m} \mathcal{U}^{\dagger}$, where $\hat{m}$ is a diagonal matrix with $2 N$ positive entries $m_{k}$ and $\mathcal{U}$ is unitary. The mass Lagrangian then takes the form

$$
\mathcal{L}_{D+M}=-\frac{1}{2} \sum_{k=1}^{2 N} m_{k} \overline{\left(n_{k L}^{\prime}\right)^{c}} n_{k L}^{\prime}+\text { H.c. }=-\frac{1}{2} \sum_{k=1}^{2 N} m_{k} \overline{\phi_{k}} \phi_{k},
$$

where $n_{L}=\mathcal{U} n_{L}^{\prime}$ and

$$
\phi_{k}=n_{k L}^{\prime}+\left(n_{k L}^{\prime}\right)^{c}=\phi_{k}^{c}
$$

with only the cross terms surviving when the $\phi_{k}$ 's are written out as in Eq. (12). Clearly, then, the physical eigenstates $\phi_{k}$ are Majorana particles. The interacting left-handed flavor eigenstates are linear combinations of the left-handed parts of these Majorana eigenstates (which do not have well-defined chirality).

Dirac neutrinos are a special case, produced if, e.g., $\mathcal{M}_{L}$ and $\mathcal{M}_{R}$ are zero. We can see this most easily in the case of the one-flavor mass matrix where $\mathcal{M}_{D}$ is simply $m$,

$$
\mathcal{M}=\left(\begin{array}{cc}
0 & m \\
m & 0
\end{array}\right) .
$$

The eigenvalues $m$ and $-m$ are obtained by diagonalizing this real matrix with a real orthogonal matrix. To get the two states with positive mass $m$ we cannot use this real matrix as $\mathcal{U}$ but instead must incorporate a phase that changes the sign of the second eigenvalue:

$$
\mathcal{U}=\left(\begin{array}{cc}
1 & i \\
1 & -i
\end{array}\right) \text {. }
$$

The phase in this matrix that mixes the degenerate Majorana states $\phi_{1}$ and $\phi_{2}$ will cause the two to cancel in the neutrino-exchange diagram via which Majorana neutrinos directly mediate $\beta \beta(0 \nu)$ decay. And since they are degenerate, orthogonal linear combinations $\chi_{1}$ $\equiv 1 / \sqrt{2}\left(\phi_{1}+i \phi_{2}\right)$ and $\chi_{2} \equiv 1 / \sqrt{2}\left(\phi_{1}-i \phi_{2}\right)$, that go into one another under charge conjugation, are also eigenstates yielding $\mathcal{L}_{D}=(-m / 2)\left(\bar{\phi}_{1} \phi_{1}+\bar{\phi}_{2} \phi_{2}\right)=-m\left(\bar{\chi}_{1} \chi_{1}+\bar{\chi}_{2} \chi_{2}\right)$. The $\chi$ 's make up the lepton-number conserving Dirac neutrino and antineutrino.

We can also use the one-flavor case to illustrate the "see-saw" mechanism, a natural explanation for the existence of light Majorana neutrinos (Gell-Mann et al., 1979; Yanagida, 1979; Mohapatra and Senjanovic 1980). Majorana mass terms for left-handed neutrinos cannot be accommodated in the standard model because those terms have different quantum numbers under $\mathrm{SU}(2)_{L}$ $\times \mathrm{SU}(2)_{R}$ than do the Dirac mass terms. But by introducing new physics (e.g., new Higgs bosons with different quantum numbers) at a very large mass scale $m_{R}$, extended models can avoid this problem. The result is often a mass matrix $\mathcal{M}$ (in the one-flavor example, for which $\mathcal{M}_{R}, \mathcal{M}_{L}$, and $\mathcal{M}_{D}$ become numbers $m_{R}, m_{L}$, and $m_{D}$ ) with $m_{R} \gg m_{D} \gg m_{L}$, where $m_{D}$, which comes from our familiar Higgs vacuum expectation value, is on the order of a typical quark or lepton mass. Diagonalization yields eigenvalues $m_{1} \approx-m_{D}^{2} / m_{R}$ and $m_{2} \approx m_{R}$. The matrix that converts from eigenstates with positive mass to flavor eigenstates is 


$$
\mathcal{U} \approx\left(\begin{array}{cc}
i & m_{D} / m_{R} \\
-i m_{D} / m_{R} & 1
\end{array}\right) .
$$

In this scheme, neutrinos that we know about are much lighter than other leptons because of the existence of other very heavy neutrinos. Even without heavy neutrinos, the fact that the only dimension-5 neutrino-mass operator in standard model fields is of Majorana form (Weinberg, 1979) leads one to expect that massive neutrinos will be Majorana particles.

In the general $N$-flavor case

$$
\nu_{l L}=\sum_{k=1}^{N} \mathcal{U}_{l k} P_{L} \phi_{k}, \quad \nu_{l R}=\sum_{k=1}^{N} \mathcal{U}_{l^{\prime} k}^{*} P_{R} \phi_{k} \quad\left(l^{\prime}=l+N\right),
$$

where $P_{L}$ and $P_{R}$ are projection operators onto states of well-defined chirality. We assume here that something like the see-saw with very large $m_{R}$ is operating so that half the eigenstates are very heavy. In that case the light eigenstates mix nearly entirely among themselves, and the $N \times N$ matrix $U$ responsible, defined to be the upperleft quarter of $\mathcal{U}$, is nearly unitary:

$$
\nu_{l L} \simeq \sum_{k=1}^{N} U_{l k} P_{L} \phi_{k}
$$

Although we have used the see-saw to arrive at Eq. (17), a mixing matrix $U$ can be defined even if the righthanded sector is light or absent.

The matrix $U$, which we introduced in Eq. (3), nominally has $N^{2}$ parameters, $N(N-1) / 2$ angles, and $N(N$ $+1) / 2$ phases. $N$ of the phases are unphysical, so that there are $N(N-1) / 2$ independent physical phases. For three active neutrino flavors, the three-phase mixing matrix can be written in the form

$$
U=\left(\begin{array}{ccc}
c_{12} c_{13} & s_{12} c_{13} & s_{13} e^{-i \delta} \\
-s_{12} c_{23}-c_{12} s_{23} s_{13} e^{i \delta} & c_{12} c_{23}-s_{12} s_{23} s_{13} e^{i \delta} & s_{23} c_{13} \\
s_{12} s_{23}-c_{12} c_{23} s_{13} e^{i \delta} & -c_{12} s_{23}-s_{12} c_{23} s_{13} e^{i \delta} & c_{23} c_{13}
\end{array}\right) \operatorname{diag}\left\{e^{i \alpha_{1} / 2}, e^{i \alpha_{2} / 2}, 1\right\}
$$

where $s_{i j}$ and $c_{i j}$ stand for the sine and cosine of the angles $\theta_{i j}, \delta$ is a Dirac phase analogous to the unremovable phase in the CKM matrix, and the other two phases $\alpha_{1}$ and $\alpha_{2}$ affect only Majorana particles. If neutrinos were Dirac particles, these two phases could be absorbed into redefinitions of the fields. The Majorana mass terms in the Lagrangian, however, are not invariant under such redefinitions. Kobzarev et al. (1980) have given a detailed discussion of the number of free parameters in the mixing matrix.

\section{RATE OF DOUBLE BETA DECAY}

The neutrino masses and mixing matrix figure prominently in neutrino-mediated $\beta \beta(0 \nu)$ decay. The rate for that process is

$$
\left[T_{1 / 2}^{0 \nu}\right]^{-1}=\sum_{\text {spins }} \int\left|Z_{0 \nu}\right|^{2} \delta\left(E_{e 1}+E_{e 2}-Q_{\beta \beta}\right) \frac{d^{3} p_{1}}{2 \pi^{3}} \frac{d^{3} p_{2}}{2 \pi^{3}},
$$

where $Z_{0 \nu}$ is the amplitude and $Q_{\beta \beta}$ is the $Q$ value of the decay. The amplitude is second order in the weak interaction and depends on the currents in the effective lowenergy semileptonic Hamiltonian $\left(\mathcal{H}_{\beta}\right)$, which we assume for the time being is purely left handed: $\mathcal{H}_{\beta}(x)$ $=\left(G_{F} / \sqrt{2}\right)\left\{\bar{e}(x) \gamma_{\mu}\left(1-\gamma_{5}\right) \nu_{e}(x) J_{L}^{\mu}(x)\right\}+$ H.c., with $J_{L}^{\mu}$ the charge-changing hadronic current. We assume as well that only the particles we know about exist, or that any others are too heavy to measurably affect $\beta \beta(0 \nu)$ decay. Then the decay is mediated solely by the exchange of three light neutrinos and the amplitude contains a lepton part (a function of $x$ and $y$ to be contracted with a similar hadron part and integrated over $x$ and $y$ ) of the form

$$
\begin{aligned}
\sum_{k} \bar{e}(x) & \gamma_{\mu}\left(1-\gamma_{5}\right) U_{e k} \phi_{k}(x) \bar{e}(y) \gamma_{\nu}\left(1-\gamma_{5}\right) U_{e k} \phi_{k}(y) \\
= & -\sum_{k} \bar{e}(x) \gamma_{\mu}\left(1-\gamma_{5}\right) U_{e k} \phi_{k}(x) \overline{\phi_{k}^{c}}(y) \gamma_{\nu} \\
& \times\left(1+\gamma_{5}\right) U_{e k} e^{c}(y)
\end{aligned}
$$

where the underbrackets indicate contraction. With our convention $\phi_{k}^{c}=\phi_{k}$, the contraction of $\phi_{k}$ with $\overline{\phi_{k}^{c}}$ turns out to be the usual fermion propagator, so that the lepton part above becomes

$$
\begin{aligned}
& -\frac{i}{4} \int \sum_{k} \frac{d^{4} q}{(2 \pi)^{4}} e^{-i q \cdot(x-y)} \bar{e}(x) \gamma_{\mu}\left(1-\gamma_{5}\right) \frac{q^{\rho} \gamma_{\rho}+m_{k}}{q^{2}-m_{k}^{2}} \gamma_{\nu} \\
& \quad \times\left(1+\gamma_{5}\right) e^{c}(y) U_{e k}^{2},
\end{aligned}
$$

where $q$ is the four-momentum transfer. The term with $q^{\rho}$ vanishes and the $m_{k}$ in the denominator can be neglected for light neutrinos, so that the amplitude is proportional to 


$$
\begin{aligned}
\left\langle m_{\beta \beta}\right\rangle \equiv\left|\sum_{k} m_{k} U_{e k}^{2}\right|= & \left.\left|m_{1}\right| U_{e 1}\right|^{2}+m_{2}\left|U_{e 2}\right|^{2} e^{i\left(\alpha_{2}-\alpha_{1}\right)} \\
& +m_{3}\left|U_{e 3}\right|^{2} e^{i\left(-\alpha_{1}-2 \delta\right)} \mid .
\end{aligned}
$$

The absolute value has been inserted for convenience, since the quantity inside it is squared in Eq. (19) and is complex if $C P$ is violated. Applying the first expression in Eq. (22) to our one-flavor example, one can see explicitly that a Dirac neutrino, which is equivalent to a degenerate Majorana pair $\phi_{1}$ and $\phi_{2}$, cannot contribute to $\beta \beta(0 \nu)$ decay because the two states would have $U_{e 1}$ $=1$ and $U_{e 2}=i$, as in Eq. (14).

To complete the calculation, one must multiply the lepton part of the amplitude by the nuclear matrix element of two time-ordered hadronic currents and integrate over $x$ and $y$. We can write the matrix element of a product of currents between initial and final nuclear states $i$ and $f$ as

$$
\begin{aligned}
\left\langle f\left|J_{L}^{\mu}(x) J_{L}^{\nu}(y)\right| i\right\rangle= & \sum_{n}\left\langle f\left|J_{L}^{\mu}(\vec{x})\right| n\right\rangle\left\langle n\left|J_{L}^{\nu}(\vec{y})\right| i\right\rangle \\
& \times e^{-i\left(E_{f}-E_{n}\right) x_{0}} e^{-i\left(E_{n}-E_{i}\right) y_{0}},
\end{aligned}
$$

where the $|n\rangle$ 's are a complete set of intermediate nuclear states, the $E_{n}$ 's are the corresponding energies, and $E_{i}$ and $E_{f}$ are the energies of the initial and final nuclei. When the two time coordinates $x_{0}$ and $y_{0}$ are ordered and integrated over, and when the exponential in Eq. (23) is combined with a similar factor from the lepton currents and the $q$ dependence of the neutrino propagator, the following factor in the amplitude results:

$$
\begin{aligned}
2 \pi & \delta\left(E_{f}+E_{e 1}+E_{e 2}-E\right) \sum_{n}\left[\frac{\left\langle f\left|J_{L}^{\mu}(\vec{x})\right| n\right\rangle\left\langle n\left|J_{L}^{\nu}(\vec{y})\right| i\right\rangle}{q^{0}\left(E_{n}+q^{0}+E_{e 2}-E_{i}\right)}\right. \\
& \left.+\frac{\left\langle f\left|J_{L}^{\nu}(\vec{x})\right| n\right\rangle\left\langle n\left|J_{L}^{\mu}(\vec{y})\right| i\right\rangle}{q^{0}\left(E_{n}+q^{0}+E_{e 1}-E_{i}\right)}\right] .
\end{aligned}
$$

We have ignored the neutrino masses, which are small compared to their momenta. The quantity $q_{0}=q$ is the energy of the virtual neutrino, to be integrated over along with the virtual momenta, and $E_{e 1}, E_{e 2}$ are the energies of the outgoing electrons. The energy $q_{0}$ is typically about an average inverse spacing between nucleons, $100 \mathrm{MeV}$ or so. This value is much larger than the excitation energy of states contributing to the decay amplitude, so the intermediate-state energies are usually replaced by an estimate $\bar{E}$ of their average value. Studies show that the resulting amplitude is not very sensitive to the choice of the average (Pantis and Vergados, 1990), and that the error it causes in the decay rate is perhaps $15 \%$. In this "closure approximation" one replaces the (now unweighted) sum over intermediate states by 1 , so that Eq. (24) above becomes

$$
\begin{aligned}
2 \pi \delta\left(E_{f}+E_{e 1}+E_{e 2}-E_{i}\right)\left(\frac{\left\langle f\left|J_{L}^{\mu}(\vec{x}) J_{L}^{\nu}(\vec{y})\right| i\right\rangle}{q^{0}\left(\bar{E}+q^{0}+E_{e 2}-E_{i}\right)}\right. \\
\left.+\frac{\left\langle f\left|J_{L}^{\nu}(\vec{x}) J_{L}^{\mu}(\vec{y})\right| i\right\rangle}{q^{0}\left(\bar{E}+q^{0}+E_{e 1}-E_{i}\right)}\right) .
\end{aligned}
$$

To go any further, we need an expression for the hadronic current $J_{L}(x)$. In the impulse approximation, thehadronic current is obtained from that of free nucleons, and the resulting one-body operator $J_{L}(x)=\Sigma_{a} \hat{O}_{a}(x) \tau_{a}^{+}$ [where the operator $\hat{O}_{a}(x)$ acts on space and spin variables of the $a$ th nucleon] is used to evaluate the matrix element between states in the initial and final nuclei. In this approximation $J_{L}^{\mu}(\vec{x}) J_{L}^{\nu}(\vec{y})=J_{L}^{\nu}(\vec{y}) J_{L}^{\mu}(\vec{x})$ because $\left(\tau_{a}^{+}\right)^{2}$ $=0$. The charge-changing hadronic current for a nucleon (i.e., the proton-bra neutron-ket matrix element of the current) is

$$
\begin{aligned}
\left\langle p\left|J_{L}^{\mu}(x)\right| p^{\prime}\right\rangle= & e^{i q x} \bar{u}(p)\left(g_{V}\left(q^{2}\right) \gamma^{\mu}-g_{A}\left(q^{2}\right) \gamma_{5} \gamma^{\mu}\right. \\
& \left.-i g_{M}\left(q^{2}\right) \frac{\sigma^{\mu \nu}}{2 m_{p}} q_{\nu}+g_{P}\left(q^{2}\right) \gamma_{5} q^{\mu}\right) \bar{u}\left(p^{\prime}\right),
\end{aligned}
$$

where $q=p^{\prime}-p, g_{V} \equiv g_{V}(0)=1, g_{A} \equiv g_{A}(0)=1.26$, conservation of the vector current tells us that $g_{M}\left(q^{2}\right)$ $=g_{M} g_{V}\left(q^{2}\right) \quad$ with $\quad g_{M} \equiv g_{M}(0) \approx 3.70 g_{V}$, and the Goldberger-Triemann relation, accurate enough for our purposes, states that $g_{P}\left(q^{2}\right)=2 m_{p} g_{A}\left(q^{2}\right) /\left(q^{2}+m_{\pi}^{2}\right)$, with $m_{p}$ and $m_{\pi}$ the proton and pion masses. The behavior with $q^{2}$ of the other coefficients can be parametrized in a simple way from experimental data:

$$
g_{V}\left(q^{2}\right)=\frac{g_{V}}{\left(1+q^{2} / \Lambda_{V}^{2}\right)^{2}}, \quad g_{A}\left(q^{2}\right)=\frac{g_{A}}{\left(1+q^{2} / \Lambda_{A}^{2}\right)^{2}},
$$

with $\Lambda_{V}^{2}=0.71(\mathrm{GeV})^{2}$ and $\Lambda_{A}^{2}=1.09(\mathrm{GeV})^{2}$. After reducing to nonrelativistic kinematics to obtain $\hat{O}(x)$, keeping all terms of $\mathcal{O}\left(1 / m_{p}\right)$ except "recoil" terms that depend on $p+p^{\prime}$ rather than $q$, and omitting secondorder terms in $E_{1,2} / q_{0}$, which takes the electron energies out of the denominators of Eq. (25), one can integrate the rate over electron phase space making the longwavelength approximation. Only $0^{+} \rightarrow 0^{+}$decay is considered so that the electrons are predominantly in $s$ states and the effect on them of the charged nucleus as they exit may be approximated via a Fermi function. The rate then takes the form given in Eq. (2):

$$
\left[T_{1 / 2}^{0 \nu}\right]^{-1}=G_{0 \nu}\left(Q_{\beta \beta}, Z\right)\left|M_{0 \nu}\right|^{2}\left\langle m_{\beta \beta}\right\rangle^{2},
$$

where $Q_{\beta \beta} \equiv E_{i}-E_{f}, G_{0 \nu}\left(Q_{\beta \beta}, Z\right)$ comes from the phasespace integral, which includes the $Z$-dependent Fermi function, and according to Šimkiovic et al. (1999) and Rodin et al. (2006), 


$$
M_{0 \nu}=\left\langle f\left|\frac{2 R}{\pi g_{A}^{2}} \int_{0}^{\infty} q d q \sum_{a, b} \frac{j_{0}\left(q r_{a b}\right)\left[h_{F}(q)+h_{\mathrm{GT}}(q) \vec{\sigma}_{a} \cdot \vec{\sigma}_{b}\right]+j_{2}\left(q r_{a b}\right) h_{T}(q)\left[3 \vec{\sigma}_{j} \cdot \hat{r}_{a b} \vec{\sigma}_{k} \cdot \hat{r}_{a b}-\vec{\sigma}_{a} \cdot \vec{\sigma}_{b}\right]}{q+\bar{E}-\left(E_{i}+E_{f}\right) / 2} \tau_{a}^{+} \tau_{b}^{+}\right| i\right\rangle .
$$

Here the nucleon coordinates are all operators that, like spin and isospin operators, act on nuclear wave functions. The nuclear radius $R$ is inserted to make the matrix element dimensionless, with a compensating factor in $G_{0 v}$. [As pointed out in Cowell (2006), errors have resulted from using different values of $R$ in $M_{0 v}$ and $G_{0 v}$.] The internucleon position vectors are defined by $r_{a b}=\left|\vec{r}_{a}-\vec{r}_{b}\right|$ and $\hat{r}_{a b}=\left(\vec{r}_{a}-\vec{r}_{b}\right) / r_{a b}$, while $j_{0}$ and $j_{2}$ are spherical Bessel functions, and

$$
\begin{aligned}
h_{F}(q) \equiv & -g_{V}^{2}\left(q^{2}\right), \\
h_{\mathrm{GT}}(q) \equiv & g_{A}^{2}\left(q^{2}\right)-\frac{g_{A}\left(q^{2}\right) g_{P}\left(q^{2}\right) q^{2}}{3 m_{p}}+\frac{g_{P}^{2}\left(q^{2}\right) q^{4}}{12 m_{p}^{2}} \\
& +\frac{g_{M}^{2}\left(q^{2}\right) q^{2}}{6 m_{p}^{2}}, \\
h_{T}(q) \equiv & \frac{g_{A}\left(q^{2}\right) g_{P}\left(q^{2}\right) q^{2}}{3 m_{p}}-\frac{g_{P}^{2}\left(q^{2}\right) q^{4}}{12 m_{p}^{2}}+\frac{g_{M}^{2}\left(q^{2}\right) q^{2}}{12 m_{p}^{2}}
\end{aligned}
$$

The terms above containing $g_{M}$ are negligible, but those with $g_{P}$ typically reduce the matrix element by about $30 \%$. In most calculations, however, even these terms are neglected, so that the matrix element takes the approximate form

$$
M_{0 \nu} \approx M_{0 \nu}^{\mathrm{GT}}-\frac{g_{V}^{2}}{g_{A}^{2}} M_{0 \nu}^{F}
$$

with

$$
\begin{aligned}
& M_{0 \nu}^{F}=\left\langle f\left|\sum_{a, b} H\left(r_{a b}, \bar{E}\right) \tau_{a}^{+} \tau_{b}^{+}\right| i\right\rangle, \\
& M_{0 \nu}^{\mathrm{GT}}=\left\langle f\left|\sum_{a, b} H\left(r_{a b}, \bar{E}\right) \vec{\sigma}_{a} \cdot \vec{\sigma}_{b} \tau_{a}^{+} \tau_{b}^{+}\right| i\right\rangle .
\end{aligned}
$$

Here the neutrino potential $H$ is defined as

$$
H(r, \bar{E}) \approx \frac{2 R}{\pi r} \int_{0}^{\infty} d q \frac{\sin q r}{q+\bar{E}-\left(E_{i}+E_{f}\right) / 2} .
$$

For later reference, we also give an approximate expression for the rate of $\beta \beta(2 \nu)$ decay, which does not depend on neutrino mass or charge-conjugation properties, and involves no neutrino propagator:

$$
\left[T_{1 / 2}^{2 \nu}\right]^{-1}=G_{2 \nu}\left(Q_{\beta \beta}, Z\right)\left|M_{2 \nu}^{\mathrm{GT}}-\frac{g_{V}^{2}}{g_{A}^{2}} M_{2 \nu}^{F}\right|^{2}
$$

where $=G_{2 \nu}\left(Q_{\beta \beta}, Z\right)$ is another phase-space factor, presented earlier in Eq. (1), and

$$
\begin{aligned}
& M_{2 \nu}^{F}=\sum_{n} \frac{\left\langle f\left|\sum_{a} \tau_{a}^{+}\right| n\right\rangle\left\langle n\left|\sum_{b} \tau_{b}^{+}\right| i\right\rangle}{E_{n}-\left(M_{i}+M_{f}\right) / 2}, \\
& M_{2 \nu}^{\mathrm{GT}}=\sum_{n} \frac{\left\langle f\left|\sum_{a} \vec{\sigma}_{a} \tau_{a}^{+}\right| n\right\rangle\left\langle n\left|\sum_{b} \vec{\sigma}_{b} \tau_{b}^{+}\right| i\right\rangle}{E_{n}-\left(M_{i}+M_{f}\right) / 2} .
\end{aligned}
$$

Nearly all the Fermi strength goes to the isobar analog state in the daughter, so that $M_{2 \nu}^{F}$ can be neglected.

We know that there are three light neutrinos with largely left-handed interactions, so it makes sense to calculate the $\beta \beta(0 \nu)$ rate that those neutrinos and interactions induce. But most theorists believe that unobserved particles and interactions exist as well. The most popular explanation of small neutrino masses is the see-saw mechanism, which implies the existence of heavy neutrinos that couple to left-handed gauge bosons. One simple extension of the standard model that gives rise to a seesaw is the "left-right" symmetric model, in which a heavy right-handed weak boson $W_{R}$ coexists alongside the familiar, and lighter, $W_{L}$. Hirsch et al. (1996a) and Prézeau et al. (2003) have given a general analysis of double beta decay in such models. We will not repeat that here, but instead examine the general question of whether we can expect physics beyond left-handed weak interactions and light Majorana neutrinos to generate double beta decay at a level that competes with Eq. (28).

Right-handed currents can cause $\beta \beta(0 \nu)$ through the exchange of both light and heavy neutrinos. The coupling of $W_{R}$ to neutrino mass eigenstates contains a factor $\mathcal{U}_{l^{\prime} i}$ (where $l^{\prime}$ labels the right-handed states with definite flavor), while the coupling of the usual $W_{L}$ contains $\mathcal{U}_{l i}$ (where $l$ labels the left-handed states), so that the exchange of light neutrinos with a right-handed $W$ involved is proportional to

$$
\sum_{k=\text { light }} m_{k} \mathcal{U}_{l^{\prime} k}^{\dagger} \mathcal{U}_{l k}
$$

As we see in our one-flavor example, Eq. (15), this quantity is $\sim m_{k} m_{D} / m_{R}$ and the amplitude is very suppressed. The largest contribution, not including the one proportional to $\left\langle m_{\beta \beta}\right\rangle$ derived above, generally comes from the exchange of heavy neutrinos, through two $W_{R}$ 's. Then there is no suppression from the mixing matrix, the neutrino propagator is roughly proportional to $1 / m_{R}$ and, crudely speaking, 


$$
Z_{0 \nu}^{\text {heavy }} \propto \frac{G_{F}^{2}}{2}\left(\frac{M_{W_{L}}}{M_{W_{R}}}\right)^{4}\left(\frac{1}{m_{R}}\right) .
$$

For light neutrino exchange, there are no $W_{R}$ 's in the dominant term, and the propagator is roughly proportional to $\left\langle m_{\beta \beta}\right\rangle /\langle q\rangle^{2}$, where $\langle q\rangle \sim 100 \mathrm{MeV}$ is a typical virtual-neutrino momentum. Then, instead of Eq. (41), we have

$$
Z_{0 \nu}^{\text {light }} \propto \frac{G_{F}^{2}\left\langle m_{\beta \beta}\right\rangle}{2\langle q\rangle^{2}}
$$

so that the two amplitudes will be approximately equal when (assuming that $M_{W_{R}} \approx m_{R}$ ) (Mohapatra 1999; Cirigliano et al., 2004),

$$
m_{R} \approx\left(\frac{M_{W_{L}}^{4}\langle q\rangle^{2}}{\left\langle m_{\beta \beta}\right\rangle}\right)^{1 / 5},
$$

which is on the order of $1 \mathrm{TeV}$ for $\left\langle m_{\beta \beta}\right\rangle \approx \sqrt{\Delta m_{\mathrm{atm}}^{2}}$. Thus if the heavy mass scale in left-right symmetric models is about a $\mathrm{TeV}$ or less, it will not be so easy to determine the mass scale of the light neutrinos from double beta decay. The same statement is true of many other hypothetical lepton-number-violating models (supersymmetry, leptoquarks, etc.) because they usually generate double beta decay in a similar way, through graphs in which heavy particles of some kind play the role of the $W_{R}$ 's and heavy neutrinos.

Neutrinoless double beta decay in extra-standard models gives rise to new nuclear matrix elements. The presence of a single right-handed lepton current causes the $q^{\rho} \gamma_{\rho}$ term in the propagator of Eq. (21) to contribute to the amplitude, giving rise to derivatives of the neutrino potential presented here or forcing one of the electrons into a $p$ state. The outgoing $p$ wave leads to a different dependence on the angle between the two emitted electrons that could in principle be exploited to distinguish between the action of right-handed currents and the neutrino mass in light neutrino exchange. But the short-range exchange of a heavy particle will not always manifest something like the $q^{\rho} \gamma_{\rho}$ term, and often the only way to distinguish such a process from neutrino-mass-induced decay is to exploit the different nuclear matrix elements that enter. Provided the matrix elements can be accurately calculated, analysis of measured lifetimes in several isotopes or to several states in the same istotope can tell you whether long or short range is responsible. Of course, as already mentioned, the accuracy with which nuclear matrix elements can be calculated is a big issue, and we discuss it later. A more detailed treatment of the matrix elements governing the various kinds of double beta decay can be found in Haxton and Stephenson (1984); Doi et al. (1985); Tomoda (1991); Šinkovic and Faessler (2002).

The implications of some popular extra-standard models for $\beta \beta(0 \nu)$ are discussed below. We close this section with two general points. First, when the lepton number is spontaneously broken, as it is in most models that result in a see-saw mass matrix, there must exist one

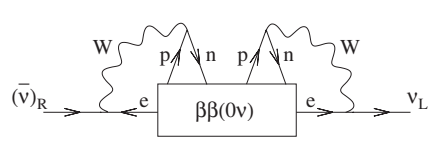

FIG. 3. Majorana propagator resulting from $\beta \beta(0 \nu)$ amplitude (Schechter and Valle, 1982).

or more zero-mass bosons, called Majorons, that could be emitted along with the two electrons in double beta decay $[\beta \beta(0 \nu, \chi)]$ (Chikashige et al., 1981; Gelmini and Roncadelli, 1981; Georgi et al., 1981). Apparently, however, it is difficult for such a process to have a very large amplitude. Second, even if some exotic lepton-numberviolating physics exists and light neutrino exchange is not responsible for the decay, the occurrence of $\beta \beta(0 \nu)$ still implies that neutrinos are Majorana particles with nonzero mass (Schechter and Valle, 1982). The reason is that any diagram contributing to the decay can be inserted into a neutrino propagator, with outgoing electron lines closed appropriately as in Fig. 3. If $\beta \beta(0 \nu)$ decay is observed, we will know for certain that neutrinos are their own antiparticles, even if the possibility of exotic physics or uncertainty in the nuclear matrix elements prevents an accurate extraction of the neutrino mass scale from observation.

\section{DOUBLE BETA DECAY AND NEW PHYSICS}

Over the past few decades much has been learned about the neutrino mixing angles and mass eigenvalues. Table I summarizes our knowledge of these neutrino parameters. These results have increased the importance of $\beta \beta(0 \nu)$ experiments; in the first subsection below, we explain why. The other subsections discuss other physics that might be revealed by $\beta \beta(0 \nu)$.

\section{A. Neutrino mass}

If neutrinos are Majorana particles, they will mediate $\beta \beta(0 \nu)$ at a rate proportional to the square of $\left\langle m_{\beta \beta}\right\rangle$, Eq. (22). The known values of the mixing-matrix elements in Eq. (18) allow us to predict the rate of $\beta \beta(0 \nu)$ under several scenarios for the neutrino's mass spectrum. If we ignore the LSND result (see Sec. IV.C) the oscillation data are consistent with only three such masses, but their spectrum can still take four possible forms:

(i) Normal hierarchy Dirac: The two masses with the smaller splitting indicated by $\delta m_{\text {sol }}^{2}$ are smaller than the third mass. The neutrinos are Dirac.

(ii) Inverted hierarchy Dirac: The two masses with the smaller splitting indicated by $\delta m_{\text {sol }}^{2}$ are larger than the third mass. The neutrinos are Dirac.

(iii) Normal hierarchy Majorana: The two masses with the smaller splitting indicated by $\delta m_{\text {sol }}^{2}$ are smaller than the third mass. The neutrinos are Majorana.

(iv) Inverted hierarchy Majorana: The two masses with the smaller splitting indicated by $\delta m_{\text {sol }}^{2}$ are 


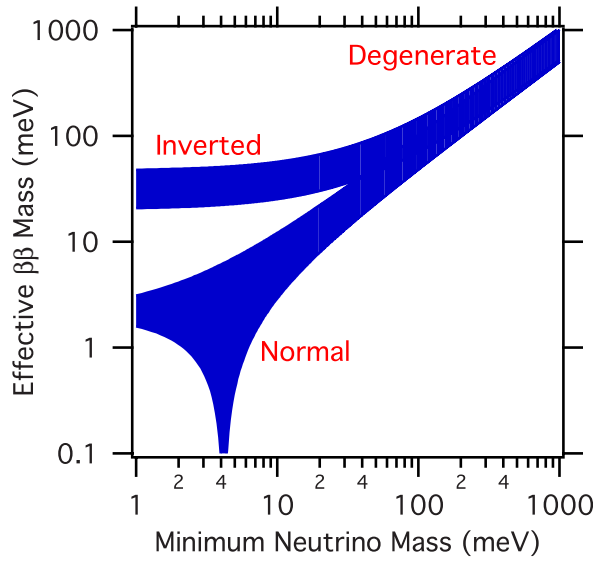

FIG. 4. (Color online) The effective Majorana mass $\left\langle m_{\beta \beta}\right\rangle$ as a function of the mass of the lightest neutrino $m_{\text {lightest }}$. In making the plot, we have used the best fit values for the parameters in Table I. The filled areas represent the range possible because of the Majorana phases and are irreducible. If one incorporates the uncertainties in the mixing parameters, the regions widen. See Bilenky et al. (2004) for an example of how the mixing parameter uncertainty affects the regions.

larger than the third mass. The neutrinos are Majorana.

In addition, since the absolute mass scale is unknown, it is possible that the differences between the three mass eigenvalues are small compared to the masses themselves. In this arrangement the neutrinos are referred to as quasidegenerate or sometimes simply as degenerate. In the normal hierarchy, the state corresponding to the largest mass contributes with a small mixing angle. Hence if the mass of the lightest state $m_{\text {lightest }}$ is small, $\left\langle m_{\beta \beta}\right\rangle$ is also small. By contrast, in the inverted hierarchy, the heavier neutrinos are large contributors to $\left\langle m_{\beta \beta}\right\rangle$.

Modern $\beta \beta$ research is exciting in part because if nature has selected possibility iv, we should be able to see $\beta \beta(0 \nu)$ with the next generation of experiments. By the same token, null experiments would rule it out, and could restrict the parameter space available for possibility iii. And if some very sensitive experiment ever saw a very small $\left\langle m_{\beta \beta}\right\rangle$, it would demonstrate that possibility iii is nature's choice. Actually certifying that possibility iv is the choice is a trickier matter, though. To see this, we show in Fig. 4 the dependence of $\left\langle m_{\beta \beta}\right\rangle$ on $m_{\text {lightest }}$. To make this plot we used the best fit values of the mixing angles $\left(\theta_{\mathrm{sol}}=33.9^{\circ}, \theta_{\mathrm{atm}}=45^{\circ}\right.$, and $\left.\theta_{13}=0^{\circ}\right)$ and the $\Delta m^{2} \mathrm{~s}$ (Table I). The figure shows, as expected, that $\left\langle m_{\beta \beta}\right\rangle$ is larger in the inverted hierarchy than in the normal one. (The plot shows regions rather than lines due to the unknown Majorana phases.) But because there is no way of measuring $m_{\text {lightest }}, \beta \beta(0 \nu)$ will not be able to distinguish the inverted hierarchy from the quasidegenerate arrangements. (Although in principle it is possible to directly measure $m_{\text {lightest }}$ in a beta decay experiment with ideal energy resolution, in practice it is not feasible.) A large $\left\langle m_{\beta \beta}\right\rangle$ will still not tell us for sure which eigenstate is the lightest. And we also will not know for sure that other TeV-scale physics is not responsible for the decay.

Other measurements can help, however. Unlike $\beta \beta(0 \nu)$, the rate of which reflects the coherent exchange of virtual neutrinos, beta decay involves the emission of real neutrinos, whose mass can alter the beta particle spectrum. The corresponding effective beta decay mass $\left\langle m_{\beta}\right\rangle$ reflects the incoherent sum of the mass terms:

$$
\left\langle m_{\beta}\right\rangle^{2}=\sum_{j} m_{j}^{2}\left|U_{e j}\right|^{2}=m_{1}^{2}\left|U_{e 1}\right|^{2}+m_{2}^{2}\left|U_{e 2}\right|^{2}+m_{3}^{2}\left|U_{e 3}\right|^{2} .
$$

In a beta decay experiment this quantity would approximate the difference between the end point of the electron spectrum and the $Q$ value. The approximation is valid as long as the energy resolution is too poor to separate individual end points due to each of the $m_{i}$. For all presently planned beta decay experiments, that is the case.

Equations (22) and (44) depend differently on the mixing angles, phases, and mass eigenvalues. If beta decay experiments find $\left\langle m_{\beta}\right\rangle, \beta \beta(0 \nu)$ measures $\left\langle m_{\beta \beta}\right\rangle$, and $M_{0 \nu}$ ever get accurate enough, they could help constrain the Majorana phases discussed below. If $\left\langle m_{\beta}\right\rangle$ yielded a result that was inconsistent with the two known $\Delta m^{2}$ 's and a measured $\left\langle m_{\beta \beta}\right\rangle$, it could demonstrate that new physics, either in the form of new particles exchanged in $\beta \beta(0 \nu)$ decay or sterile neutrinos that mix with the three active neutrinos, is at play.

We should note that the neutrino contribution to the mass density $\left(\Omega_{v}\right)$ of the Universe (Hannested, 2003) constrains a third combination of the neutrino masses:

$$
\Omega_{\nu} h^{2}=\frac{\Sigma}{92.5 \mathrm{eV}},
$$

where

$$
\Sigma=\sum_{j} m_{j}=m_{1}+m_{2}+m_{3} .
$$

Since no experiment measures the mass eigenstates directly, effective neutrino mass measurements, coupled with the oscillation measurements, are all required to determine a complete set of the parameters describing neutrinos.

\section{B. The Majorana phases}

The elements of the mixing matrix may contain many phases, but only a small number have any physical consequence. In a $3 \times 3$ mixing matrix describing Dirac neutrinos, all but one phase (the so-called Dirac phase $\delta$ ) can be absorbed into a redefinition of the neutrino and antineutrino fields. If neutrinos are Majorana, the phases of each neutrino and antineutrino pair are correlated and fewer phases can be eliminated in this way. For the $3 \times 3$ case, two additional phases [the Majorana phases $\alpha_{1}, \alpha_{2}$ of Eq. (18)] are allowed. Any of these phases can result in $C P$ violation if its value differs from 
a multiple of $\pi$. In Fig. 4, the borders of the shaded regions correspond to phases that are multiples of $\pi$, resulting in $C P$ conservation. The shaded regions between the borders correspond to $C P$ violation. By comparing measurements of $\Sigma$ or $\left\langle m_{\beta}\right\rangle$ to $\left\langle m_{\beta \beta}\right\rangle$, one might be able to demonstrate that $C P$ is violated in the lepton sector.

The Dirac phase can lead to observable effects in oscillation experiments, whereas the Majorana phases that appear in Eq. (22) have no effect in those experiments. To see this, note that the transition amplitude for oscillations from a neutrino of flavor $l$ to flavor $j$ is

$$
Z\left(\nu_{l} \rightarrow \nu_{j}\right)=\sum_{k} U_{j k} U_{l k}^{*} e^{-2 i m_{k}^{2} L / 4 E},
$$

where $L$ and $E$ are the distance traveled and energy, respectively, of the $\nu$. The Majorana phases in the diagonal matrix of Eq. (18) cancel with their complex conjugates in the product $U U^{*}$.

Many have suggested that the Majorana phases might be observable if precise values of $\left\langle m_{\beta \beta}\right\rangle$ and $\left\langle m_{\beta}\right\rangle$ could be compared (Abada and Bhattacharyya, 2003; Pascoli and Petcov, 2003; Sugiyama, 2004). Elliott and Engel (2004) have provided a graphical example of how the measurements from oscillations, $\beta \beta(0 \nu)$, beta decay, and cosmology might be combined to learn about possible values for the phases of Eq. (22). But any attempt would have to contend with the problem that there are two such phases but only one known experimental measurement that is sensitive to them. With only $\beta \beta(0 \nu)$ as a probe, no unambiguous measurement of both $\alpha$ 's is possible. Although leptogenesis depends on $C P$ violation in the lepton sector (Fukugita and Yanagida, 1986; Buchmüller et al., 2002), it will not be easy to use cosmological measurements to help quantify the $\alpha$ 's, because the relation between $C P$ violation in the decay of heavy Majorana neutrinos in the early Universe and the phases in the light-neutrino mixing matrix is model dependent.

If $\theta_{13}$ is zero, however, only one of the $\alpha$ 's will contribute to $\left\langle m_{\beta \beta}\right\rangle$. This might allow that $\alpha$ to be determined from measurements of $\beta \beta(0 \nu)$. There is still a problem, though: extracting information about $\alpha$ will require the calculation of $M_{0 \nu}$ with an accuracy that is currently out of reach (Barger et al., 2002; Elliott, 2006).

\section{Sterile neutrinos}

The LSND (Aguilar et al., 2001) neutrino-oscillation result indicates a $\Delta m^{2}$ scale of $\sim 1 \mathrm{eV}^{2}$. A value this large cannot be incorporated into a three-neutrino mixing scheme along with the atmospheric and solar scale $\Delta m^{2}$ values. This inconsistency is sometimes called the three $\Delta m^{2}$ problem: with three neutrino masses there are only two mass differences. One approach to solving this problem is to add some number of light sterile neutrinos to the three active neutrinos known to exist.

Light sterile neutrinos might seem unlikely, but can be produced by the see-saw mechanism (Gell-Mann et al., 1979; Yanagida, 1979; Mohapatra and Senjanovic, 1980) if a symmetry makes the Majorana mass matrix singular (Chun et al., 1998; Chikira et al., 2000; Goldman et al., 2000; Stephenson et al., 2005). Goswami and Rodejohann (2006) have reviewed the consequences of light steriles of various kinds for $\beta \beta$. In particular they discussed a model, originally suggested by Sorel et al. (2004), that adds two sterile neutrinos. One solution reproduces the LSND data while still matching the null results from other short-baseline-accelerator neutrino oscillation experiments and does not violate constraints from cosmology. This solution $\left(\Delta m_{41}^{2}=0.46 \mathrm{eV}^{2}, \Delta m_{51}^{2}\right.$ $\left.=0.89 \mathrm{eV}^{2}, \quad U_{e 4}=0.090, U_{e 5}=0.125\right)$ would provide a maximum additional contribution to $\left\langle m_{\beta \beta}\right\rangle$ of $\sim 20 \mathrm{meV}$ if $m_{1}$ is small. The MiniBooNE experiment (Bazarko, 2000; Aguilar-Arevalo et al., 2007) has found no evidence for the two-flavor oscillation model as an explanation for the LSND result, although the experiment sees a low-energy excess that is so far unexplained. Whether models with three active neutrinos and two or more sterile neutrinos can explain both sets of data is still being investigated [e.g., Maltoni and Schwetz (2007)]. Sterile neutrinos, however, have the virtue of saving the heavy element nucleosynthesis process in the neutrino-driven wind behind a supernova shock (Fetter et al., 2003), and are not ruled out in general by any experiments.

\section{Alternative mechanisms}

If $\beta \beta(0 \nu)$ is observed, it will, as discussed in Sec. III, demonstrate that the neutrino mass matrix has a nonzero Majorana component (Schechter and Valle, 1982). But, as discussed earlier, this does not imply that the decay rate is proportional to the square of $\left\langle m_{\beta \beta}\right\rangle$, as it would be if the light-neutrino mass were the driving mechanism. After any observation of $\beta \beta(0 \nu)$ therefore it would become important to find an experimental signature that isolates the underlying physics. The decay rate for $\beta \beta(0 \nu)$ depends on a lepton number violating parameter (LNVP)- $\left\langle m_{\beta \beta}\right\rangle$ in the case of light neutrino exchange produced by left-handed currents - that will be different for each possible mechanism. In addition, the nuclear matrix element may also depend on the exchange mechanism.

A number of suggestions for potential signatures of the underlying physics have appeared. As noted above, if the weak interaction includes right-handed currents, the left-handed virtual neutrino could couple to the absorbing neutron without a helicity flip. Doi et al. (1985) suggested the use of kinematic distributions to discern right-handed currents from light-neutrino-mass contributions and Ali et al. (2006) discussed the use of the angular distribution to distinguish the left-right symmetric model from other possibilities. Tomoda (2000) proposed examining the ratio of rates to the $2^{+}$excited state to that to the ground state in the same isotope as a signature of right-handed currents.

Many alternative exchange mechanisms involve heavy exchange particles $(\gtrsim 1 \mathrm{TeV})$, such as heavy neutrinos 
(Hirsch et al., 1996a), leptoquarks (Hirsch et al., 1996b), supersymmetric particles (Hirsch et al., 1996c; Vergados, 2002), and scalar bilinears (Klapdor-Kleingrothaus and Sarkar, 2003). As noted already, electron kinematics will not in general depend on the heavy particle exchanged (Prézeau et al., 2003). Šimkovic and Faessler (2002) showed that the relative rates of the decay to the first excited $0^{+}$state and the ground state might distinguish among light-neutrino exchange, the heavy-neutrino exchange, and supersymmetric-particle exchange. Prézeau (2006) estimated the relative contributions to $\Gamma^{0 v}$ from light neutrinos and heavy particle exchange.

Some models lead to complicated expressions for decay rates. Singlet neutrinos in extra dimensions can mediate $\beta \beta(0 \nu)$ (Bhattacharyya et al., 2003), but the Kaluza-Klein spectrum of neutrino masses spans values from much less than to much greater than the nuclear Fermi momentum. One cannot therefore factor the decay rate into a product of nuclear and particle factors. Futhermore, the decay rate depends on unknown parameters such as the brane-shift parameter and the extra-dimension radius. Finally, mass-varying neutrinos might lead to decay rates that depend on the density of the matter in which the process occurrs (Kaplan et al., 2004).

Cirigliano et al. (2004) recognized the potential of using $\mu \rightarrow e$ and $\mu \rightarrow e \gamma$ in combination with $\beta \beta(0 \nu)$ to decide whether light-neutrino exchange is the dominant mechanism. Certain supersymmetric particles and heavy Majorana neutrinos could be produced at the CERN Large Hadron Collider. Seeing these particles, or not seeing them, could help us determine what is responsible for $\beta \beta(0 \nu)$.

\section{CALCULATING NUCLEAR MATRIX ELEMENTS}

\section{A. Nuclear-structure theory}

The better we can calculate the nuclear matrix elements that govern double beta decay, the more accurately we can extract an effective neutrino mass, or a heavy-particle mass. Some have tried to minimize or play down the uncertainty in the matrix element in an attempt to strengthen claims regarding the precision with which $\left\langle m_{\beta \beta}\right\rangle$ can be determined. In contrast, physicists in other fields have occasionally exaggerated the uncertainty to downplay the impact $\beta \beta(0 \nu)$ would have regarding conclusions about neutrino mass. Here we try to assess this complex issue as objectively as possible. To begin, we review the accomplishments, difficulties, and main lines of inquiry in the theory of nuclear structure, which is currently in an exciting period.

There are a large number of nuclei and many phenomena to observe in each one. Nuclear-structure theory has tended to concentrate on properties that are easiest to measure: spectra, electromagnetic transition rates, and cross sections for the scattering of electrons or hadrons. Traditional nuclear-structure theory divides the chart of nuclides into different regions-some in which the nuclei are deformed, some in which they are spheri- cal, others in which the behavior is more complicatedand adopts different models for each kind of nucleus. Increased computing power is allowing us to gradually abandon these models, which usually consider the dynamics of only one or a few nucleons explicitly, lumping the rest into a collective core with only a few degrees of freedom. Calculations now are increasingly ab initio, attempting to solve the many-body Schrödinger equation directly from empirical two- and three-nucleon interactions, which are obtained from fits to $N N$ phase shifts and energy levels in the triton and ${ }^{3} \mathrm{He}$. Monte Carlo techniques now yield accurate wave functions with such interactions in nuclei as heavy as ${ }^{12} \mathrm{C}$ [see Pieper and Wiringa (2001), and references therein], and controlled approximation schemes that treat all nucleons with the same interactions [e.g., the no-core shell model (Navratil et al., 2000) and the coupled-clusters approach (Kowalski et al., 2004; Wloch et al., 2005)] currently work well for bound-state energies in nuclei up to about ${ }^{16} \mathrm{O}$, with the closed-shell nucleus ${ }^{40} \mathrm{Ca}$ in range.

Unfortunately, these calculations are not likely to reach atomic numbers relevant to $\beta \beta$ soon, and even if they did, would focus first on spectra and other observables generated by one-body operators before considering the difficult two-body decay operator discussed in Sec. III. The other main line of structure theory, however, is geared towards systematic (though less accurate) predictions in arbitrarily heavy nuclei. Its framework is mean-field theory (treating all nucleons) and extensions such as the random phase approximation (RPA), restoration of symmetries artificially broken by the mean field, and the generator-coordinate method (Bender et al., 2003). These techniques, which can involve modifying the equations in ways that improve phenomenology but at first sight appear dubious, are closely related to density-functional theory, which leads naturally to similar mean-field-like equations and has been successful in electronic systems. But as in lighter nuclei, the initial focus is on nuclear spectra, with some attention to simple transitions. Though other observables can be calculated, it can be difficult to judge the accuracy of the results, because systematic data with which to test calculations are not available.

These two lines of research-ab initio and mean-field calculations-are both developing rapidly and naturally absorb most of the energy of the nuclear-structuretheory community. Relatively few researchers focus on double beta decay, which involves complicated operators in structurally complex nuclei such as ${ }^{76} \mathrm{Ge}$, and for good reason: there are so many more general problems that have frustrated theory for years but are now becoming solvable. As a result, the models and techniques that have been applied to double beta decay are more limited in scope and power than they might be. They fall primarily into two categories, the quasiparticle random phase approximation (QRPA) and the shell model. We discuss each below and try to assess the accuracy of the corresponding results before looking at prospects for improved calculations. 


\section{B. QRPA}

The neutron-proton QRPA, first used by Hableib and Sorensen (1967), is an extension of the random phase approximation, a technique primarily for calculating the energies and excitation strengths of collective states. In calculations of double beta decay, a number of approximations are made even before the QRPA is applied. Most nuclear-structure calculations add to the Hamiltonian, a one-body potential amd mean field, which is diagonalized first to provide a single-particle basis in which to treat the two-body (and in principal threebody) Hamiltonian that comes from $N N$ and $N N N$ data. In a complete calculation, the effects of the one-body potential are subtracted off later. In most nuclearstructure calculations, however, the potential is retained and the single-particle basis truncated. In double beta decay QRPA calculations, the "model space" typically includes states within 10 or $20 \mathrm{MeV}$ of the Fermi surface. The energies of these states (or, equivalently, the one-body potential) are adjusted to reproduce properties of states with a single nucleon outside a closed shell.

The two-body interaction is also not treated exactly, again even before application of the QRPA. It is possible in principle to construct an effective Hamiltonian and effective transition operators that, for low-lying states, have the same eigenvalues and matrix elements in the truncated single-particle space as does the full Hamiltonian in the full Hilbert space. In applications of the QRPA, the effective interaction is usually approximated by a $G$ matrix (Hjorth-Jensen et al., 1995), which takes into account the exclusion of very high-lying levels but not those just above the model space or those below, which are assumed to contain an inert core. Then certain parts of the interaction and operator are scaled to reproduce data: all $0^{+}$matrix elements are sometimes multiplied by about 1.1 so that when the interaction is treated in the BCS approximation (the starting point of the QRPA) they reproduce experimental odd- $A$-even- $A$ mass differences. The parameter $g_{A}$ that is squared in the double beta operator is often taken to be 1.0 rather than 1.26, and short-range correlations responsible for converting the bare Hamiltonian into a $G$ matrix must be inserted explicitly (although approximately) into the operator's two-body matrix elements. Other more dramatic adjustments take place in the application of the QRPA itself.

To understand the logic behind the neutron-proton QRPA, we first remove the $Q$, addressing the simpler charge-changing RPA. The two-body operator that governs (in the closure approximation) the decay, in secondquantized form, can be written $\hat{O}=\sum_{i j k l} O_{i j k l} p_{i}^{\dagger} p_{j}^{\dagger} n_{k} n_{l}$, where $p^{\dagger}$ creates a proton, $n$ destroys a neutron, and the coefficients $O_{i j k l}$ are two-body matrix elements. One can rewrite this operator as a sum of products of one-body operators $\hat{O}=\sum_{i j k l} O_{i j k l}\left(p_{i}^{\dagger} n_{k}\right)\left(p_{j}^{\dagger} n_{l}\right)$. In the chargechanging RPA the one-body $p^{\dagger} n$ and $n^{\dagger} p$ operators are replaced by operators with the same quantum numbers but boson commutation rules, both in the decay opera- tor and the Hamiltonian. After the "bosonized" Hamiltonian is diagonalized to obtain a representation of the intermediate-nucleus states as a set of "one-phonon" states, the bosonized one-body operators contained in the decay operator then connect those states with the mother and daughter ground states, which are "phonon vacua." (Actually there are two sets of intermediate states, one corresponding to excitations of the mother vacuum and one to excitations of the daughter. The sets are not identical, a fact that has so far been taken into account only approximately.) This "quasiboson" approximation is expected to work best for strongly excited intermediate states, where excitations with different single-particle quantum numbers contribute coherently. When the intermediate states are summed over, the result is the ground-state to ground-state double beta decay matrix element.

The QRPA differs from the above only in that pairing between like particles is taken into account through BCS theory, so that the mother and daughter nuclei are now "quasiparticle phonon" vacua. They include correlations by breaking number conservation and have the correct number of neutrons and protons only on average. The smearing of the Fermi surface induced by pairing has a large effect on the matrix elements, which actually vanish for $\beta \beta(2 \nu)$ in the absence of pairing because the transition to the daughter ground state is Pauli blocked.

Two-quasiparticle states, the creation operators for which are now the bosonized objects, contain some components that are particle (proton)-hole (neutron) excitations and others that are particle-particle or hole-hole excitations (based on nuclei with two more or less particles than the one we are actually interested in). The interaction has matrix elements in both channels, and these matrix elements are usually treated as independent because a large change in, e.g., a single particleparticle matrix element translates into small changes in all particle-hole matrix elements. The particle-hole matrix elements are generally left alone because their primary effect is on the giant Gamow-Teller resonance, which is collective and reproduced pretty well by the QRPA. The particle-particle matrix elements, by contrast, affect low-lying states with much less strength, and are multiplied by a parameter traditionally called $g_{p p}$ that takes values between 0.8 and 1.2. This parameter turns out to have a large effect on double beta decay, particularly the $2 \nu$ variety and particularly in the QRPA (Vogel and Zirnbauer, 1986; Engel et al., 1988). It is needed because the QRPA simplifies the particleparticle correlations in a way that must be compensated. The parameter is usually adjusted to reproduce related observables such as $\beta^{+}$or $\beta \beta(2 \nu)$ rates, which also depend on it strongly. Although adjusting the parameter increases $\beta^{+}$rates, for example, to better agree with experiment, it appears to concentrate the strength of the $\beta^{+}$operator at too low an energy. And if the parameter is increased past its best value, an unphysical deuteron condensate becomes lower in energy than the BCS-like quasiboson vacuum, causing the QRPA to break down 
completely. Some of this problem is due to the artificial separation of particle-particle correlations into static, correlations associated with isovector pairing that are in the BCS quasiparticle vacuum, and dynamic correlations, associated with isoscalar pairing (i.e., neutronproton particle-particle interactions in the $L=0$ channel) that appear in QRPA through the parameter $g_{p p}$ as a correction to the quasiparticle vacuum. A satisfactory treatment of both kinds of pairing on the same footing, something we are still awaiting in QRPA-like methods, would help.

In the meantime, much of the QRPA work has revolved around two questions: (i) How, besides the better treatment of pairing just mentioned, can the artificial sensitivity of the QRPA to $g_{p p}$ be reduced, and (ii) which observables should fix $g_{p p}$ [beta decay, $\beta \beta(2 \nu)$, etc.] and how accurate will the resulting $\beta \beta(0 \nu)$ matrix elements be? The first issue has been the subject of a large number of papers. One approach is second QRPA (Raduta et al. 1991; Stoica and Klapdor-Kleingrothaus, 2001), in which the fact that the quasiboson approximation is merely the first term in an exact expansion is exploited by calculating the effects of the next term. Another very popular approach is renormalized QRPA (RQRPA) (Hara, 1964; Rowe, 1968; Toivanen and Suhonen, 1995). The quasiboson approximation is equivalent to replacing commutators by their ground-state expectation values with the ground states treated in the BCS approximation. The RQRPA uses the QRPA ground states instead of BCS states to evaluate the commutators. Because the commutators in turn help fix the ground states, the two are evaluated self-consistently. A variant of this approach is the full RQRPA, in which the effects of isovector $n p$ particle-particle interaction, artificially strengthened to account implicitly for isoscalar pairing that underlies the sensitivity to $g_{p p}$, are included in the BCS calculation that defines the quasiparticles as well as in the subsequent QRPA calculation (Schechter et al., 1996; Šimkovic et al., 1997). [Isovector $n p$ pairing was first introduced in this way in the unrenormalized QRPA (Cheoun et al., 1993; Pantis et al., 1996).] Another extension is the self-consistent RQRPA (SCQRPA) (Bobyk et al., 2001), in which the occupation numbers in the RQRPA ground state are fed back into the BCS approximation and the BCS-RQRPA sequence iterated until the BCS and RQRPA ground states are consistent. All these methods reduce the dependence of the matrix elements on the strength of the neutron-proton pairing interaction.

On the other hand, all these calculations also leave out correlations, and the profusion of related methods, none clearly superior to the rest, is confusing. Some people have even begun to treat the calculations as a statistical sample from which to extract an error bar on the calculated matrix elements (Bahcall et al., 2004b), reaching the conclusion that the matrix elements are only known to within an order of magnitude. Elliott and Engel (2004) tabulated results for ${ }^{76} \mathrm{Ge}$ and discussed why a statistical analysis is not a good idea: many of the calculations are explicitly preliminary or make no attempt to reproduce related data. Here we note that Rodin et al. (2003, 2006), addressing issue (ii) above, argued that if the strength of the pairing interaction is adjusted to fit experimental mass differences and (especially) if $g_{p p}$ is then adjusted to reproduce measured $\beta \beta(2 \nu)$ rates, then almost all QRPA-like calculations, independent of their choice of number of single-particle levels, interaction, quenching of $g_{A}$, etc., give the same results to within about $30 \%$. If not, then they have either not included the induced-pseudoscalar terms in the nucleon current or neglected to add short-range correlations to the wave functions. Though we have no way of knowing for sure that any of the calculations give results that are right, it is comforting to think that they agree with one another. Not everyone is reassured, however, for two reasons. First, the claim that agreement with measured $\Gamma_{2 \nu}$ is more important than agreement with other observables, such as single beta decay rates from the lowest lying states in the intermediate nucleus, can be disputed (Suhonen, 2005). When single beta decay is used to adjust parameters, the resulting $\Gamma_{0 \nu}$ are different because the QRPA is not able to reproduce both the beta decay and the $\beta \beta(2 \nu)$ at the same time. Second, the size of matrix-element quenching by short-range correlations, which affect $\beta \beta(0 \nu)$ but not $\beta \beta(2 \nu)$ (for which the operator has no radial dependence), is under debate. Most have used the phenomenological correlation function of Miller and Spencer (1976), but recent papers (Kortelainen and Suhonen, 2007; Kortelainen et al., 2007) argued that more realistic procedures, e.g., the unitary operator correlation method [see, e.g., Roth et al. (2005)], produce substantially less quenching. Until these issues are resolved-and the short-rangecorrelations issue clearly can be with more theoretical work (see, e.g., Šimkovic et al., 2007)—it is hard to say that the QRPA gives unique predictions for the matrix elements (and therefore to the value of $\left\langle m_{\beta \beta}\right\rangle$ extracted from an experiment) to better than a factor of 2 or so.

There are also systematic deficiencies in the way the QRPA has been applied, in addition to the obvious simplification inherent in the method itself, that may cause errors. Almost all calculations so far have assumed that the ground states are spherical, when we know in many cases, e.g., ${ }^{76} \mathrm{Ge}$, that they are not. Some preliminary work on $\beta \beta(2 \nu)$ with a deformed QRPA exists (Šimkovic et al., 2003; Alvarez-Rodriguez et al., 2006), but $\beta \beta(0 \nu)$ remains uninvestigated. With today's computers, however, there is no reason not to use a deformed QRPA. The inclusion of the continuum is another feature that has been implemented just once, in a preliminary way (Rodin and Faessler, 2006). Here too the state of the art in nuclear structure is more advanced than what is being applied to double beta decay. In meanfield-based nuclear structure studies, continuum versions of the QRPA that treat all nucleons (so that there is no inert core) have been applied many times, and versions of these treatments that include deformation are beginning or about to appear (Hagino et al., 2004). A Skyrme- 
Hartree-Fock-Bogoluiobov + continuum QRPA calculation of $\beta \beta(0 \nu)$ which is clearly possible even with deformation included, would be worthwhile. Calculations of single beta decay rates in that formulation, although not yet with deformation, have been around for some time [see, e.g., Engel et al. (1999)]. Perhaps one reason this more complete calculation has not been done for double beta decay is that it would still treat isovector and isoscalar pairing differently, and as a result would still show sensitivity to $g_{p p}$. What is really needed to lessen reliance on parameter tuning is a more unified treatment of particle-particle correlations. The shell model, although it has its own set of difficulties, does provide that.

\section{Shell model}

The nuclear shell model was recently reviewed by Caurier et al. (2005). Compared with the QRPA it has only one disadvantage: the (much) smaller number of single-particle states that can be included. But because the number is smaller, one can include correlations of arbitrary complexity within the single-particle space. In addition, it is possible to calculate essentially any spectroscopic observable. In the QRPA, by contrast, only energies of or transitions from ground states can really be reliably calculated because the correlations necessary to describe configurations more complicated than a twoquasiparticle excitation of the ground state are not included.

Because of its sophisticated treatment of correlations, a good shell-model calculation is harder to carry out than a QRPA calculation. First, as in the QRPA, the energies of single-particle states, which usually are assumed to have harmonic-oscillator wave functions, must be fit to spectra in closed-shell +1 nuclei and an effective interaction appropriate to the model space must be constructed. Typically something like a $G$ matrix is a starting point but with many extra terms subsequently added and fit to a wide range of energies and transition rates in nuclei near the beginning or end of the shell, where Hamiltonian matrices are relatively small. In the nuclei of interest, the Hamiltonain matrices are much largernowadays up to $10^{9} \times 10^{9}$ or even larger-and the Lanczos method is often used to diagonalize them. The transition operator is modified by inserting the effects of short-range correlations, again as in the QRPA. The axial vector coupling constant is sometimes quenched, but not always.

The first modern shell-model calculations of $\beta \beta$ decay are from Haxton and Stephenson (1984), and references therein. Only a few truly large-scale shell model calculations have been performed. The heavy deformed $\beta \beta$ nuclei, ${ }^{238} \mathrm{U}$, and ${ }^{150} \mathrm{Nd}$, for example, require bases that are too large to expect real accuracy from the shell model. ${ }^{1}$ Realistic work has thus been restricted to ${ }^{48} \mathrm{Ca},{ }^{76} \mathrm{Ge}$,

\footnotetext{
${ }^{1}$ Psuedo-SU(3)-based truncations have been used for these nuclei (Hirsch et al., 1995).
}

${ }^{82} \mathrm{Se}$, and ${ }^{136} \mathrm{Xe}$ (Caurier et al., 1996; Horoi et al., 2007) [and now, though still unpublished (Caurier, Nowacki, and Poves, 2007; Caurier et al., 2007) in ${ }^{116} \mathrm{Cd},{ }^{128} \mathrm{Te}$, and ${ }^{130} \mathrm{Te}$ as well]. Less comprehensive calculations have been carried out in several other nuclei (Suhonen et al., 1997).

The largest-scale shell-model calculations tend to produce matrix elements that are a bit smaller than those from QRPA, by factors of up to 2 or 3. In addition, recent unpublished results (Caurier, Nowacki, and Poves, 2007; Caurier et al., 2007) appear to differ by $50 \%$ or more from earlier published results by the same authors (Caurier et al., 1996), who are essentially the only people working on the problem in a comprehensive way. Some of the uncertainties, e.g., the quenching of $g_{A}$ and the effects of short-range correlations, are the same ones that afflict the QRPA; others are connected with truncation and determination of the effective Hamitonian.

The shell-model calculations can be improved in the short term, even without going to larger spaces [which is in fact possible via, e.g., the factorization method of $\mathrm{Pa}-$ penbrock and Dean (2003)]. Perhaps the most important step that could be taken now is a more systematic treatment of the effective decay operator appropriate for the shell-model space. Perturbative corrections to the operator that account for the finite model-space size can be calculated, although perturbation theory in the residual two-body interaction does not always converge (HjorthJensen et al., 1995). Its use would nonetheless give us a better idea about the size of corrections to the naive renormalization (quenching of $g_{A}$ and artificial shortrange correlations) that is the current state of the art. Exploratory calculations in a solvable model indicate that the corrections may be significant (Engel and Vogel, 2004). Though perturbative corrections to the GamowTeller operator have been evaluated (Siiskonen et al., 2001), nobody has looked at two-body operators beyond the Hamiltonian.

\section{Prospects for the future}

Most good calculations give the same result for a given matrix element to within a factor of 2 or 3, usually less. Assuming that there is no many-body physics that affects the result but is systematically absent from all calculations, we can take that range to approximately represent the level of uncertainty in our knowledge of the matrix element. What are the prospects for reducing that uncertainty?

In the short term we can make progress by being more careful-by quantifying uncertainty in the weak nucleon current, form factors, short-range correlations, quenching, etc. The size of short-range-correlation effects is currently in dispute, but structure theorists now know enough about such effects to resolve the debate. In the medium term, the best hope is a better shellmodel calculation. Here what we really need is progress in constructing effective operators, both the Hamiltonian and the decay operator. Recent years have seen 


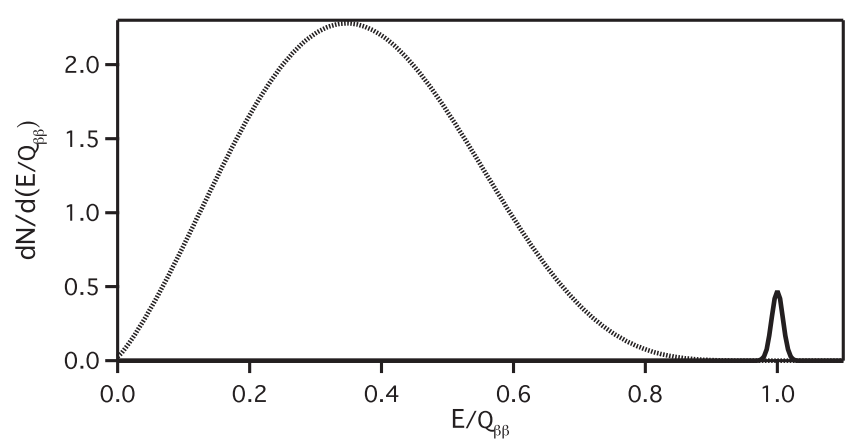

FIG. 5. The distribution of the sum of electron energies for $\beta \beta(2 \nu)$ (dotted curve) and $\beta \beta(0 \nu)$ (solid curve). The curves were drawn assuming that $\Gamma_{0 \nu}$ is $1 \%$ of $\Gamma_{2 \nu}$ and for a $1-\sigma$ energy resolution of $2 \%$.

dramatic progress in our ability to compensate for highmomentum physics that is cut out [see, e.g., Bogner et al. (2003)], but reliably correcting for low energy excitations such as core polarization is a longstanding problem. Partial summation of diagrams, a tool of traditional effective-interaction theory, is helpful but apparently not foolproof.

In the long term these issues will be solved. As already mentioned, the coupled-cluster approximation, an expansion with controlled behavior, is being applied in nuclei as heavy as ${ }^{40} \mathrm{Ca}$. With enough work on three- and higher-body forces, on center-of-mass motion, and on higher-order clusters, we should be able to handle ${ }^{76} \mathrm{Ge}$. The time it will take is certainly not short, but may be less than the time it will take for experimentalists to see neutrinoless double beta decay, even if neutrinos are indeed Majorana particles and the inverted hierarchy is realized. And the pace of theoretical work will increase dramatically if the decay is seen. Observations in more than one isotope will only make things better. Our opinion is that the uncertainty in the nuclear matrix elements in no way reduces the attractiveness of double beta decay experiments. Given enough motivation, theorists are capable of more than current work seems to imply.

\section{EXPERIMENTAL ASPECTS}

\section{A. Background and experimental design}

Double beta decay experiments are searching for a rare peak (see Fig. 5) upon a continuum of background. Observing this small peak and demonstrating that it is truly $\beta \beta(0 \nu)$ is a challenging experimental design task. The characteristics that make an ideal $\beta \beta(0 \nu)$ experiment have been discussed (Elliott and Vogel, 2002; Zdesenko 2002; Elliott, 2003). Although no detector design has been able to incorporate all desired characteristics, each includes many of them. (Section VII.C describes the various experiments.) Here we list the desirable features:

- The detector mass should initially be large enough to cover the degenerate mass region $(100-200 \mathrm{~kg}$ of iso- tope) and be scalable to reach the inverted-hierarchy scale region ( $\approx 1$ ton of isotope).

- The $\beta \beta(0 \nu)$ source must be extremely low in radioactive contamination.

- The proposal must be based on a demonstrated technology for the detection of $\beta \beta$.

- A small detector volume minimizes internal backgrounds, which scale with the detector volume. It also minimizes external backgrounds by minimizing the shield volume for a given stopping power. A small volume is easiest with an apparatus whose source is also the detector. Alternatively, a very large source may have some advantage due to selfshielding of a fiducial volume.

- Though expensive, the enrichment process usually provides a good level of purification and also results in a (usually) much smaller detector.

- Good energy resolution is required to prevent the tail of the $\beta \beta(2 \nu)$ spectrum from extending into the $\beta \beta(0 \nu)$ region of interest. It also increases the signalto-noise ratio, reducing the background in the region of interest. Two-neutrino double beta decay as background was analyzed by Elliott and Vogel (2002).

- Ease of operation is required because these experiments usually operate in remote locations and for extended periods.

- A large $Q_{\beta \beta}$ usually leads to a fast $\beta \beta(0 \nu)$ rate and also places the region of interest above many potential backgrounds.

- A relatively slow $\beta \beta(2 \nu)$ rate also helps control this background.

- Identifying the daughter in coincidence with the $\beta \beta$ decay energy eliminates most potential backgrounds except $\beta \beta(2 \nu)$.

- Event reconstruction, providing kinematic data such as opening angles and individual electron energies, can reduce background. These data might also help distinguish light- and heavy-particle exchange if a statistical sample of $\beta \beta(0 \nu)$ events is obtained.

- Good spatial resolution and timing information can help reject background processes.

- The nuclear theory is better understood in some isotopes than others. The interpretation of limits or signals might be easier for some isotopes.

Historically, most $\beta \beta$ experiments have faced $U$ and Th decay-chain isotopes as their limiting background component. A continuum spectrum arising from Compton-scattered $\gamma$ rays, $\beta$ rays (sometimes in coincidence with internal conversion electrons), and $\alpha$ particles from the naturally occurring decay chains can overwhelm any hoped for peak from the $\beta \beta(0 \nu)$ signal. This continuum is always present because $U$ and Th are present as contaminants in all materials. The level of contamination, however, varies from material to mate- 
rial and even within different production batches of the same material. Over the years, progress has been made identifying the level of contamination in materials that compose detectors and their shields. Once the location of the background-causing contaminant was determined, either cleaner materials were substituted or purification techniques were developed. As a result, detectors are now fabricated from amazingly pure components, some with activities as low as $1 \mu \mathrm{Bq} / \mathrm{kg}$ or less.

As $\beta \beta(0 \nu)$ experiments reach for the $\left\langle m_{\beta \beta}\right\rangle$ scale implied by the atmospheric neutrino oscillations, the rate of the signal is anticipated to be a few counts/(tonne yr). To observe such a small peak superimposed on a continuum of background, the background underlying the peak will need to be 1 count/ (tonne yr) or less. This will require unprecedented low levels of $U$ and $T h$ within the experimental apparatus. In fact, the required levels of assay sensitivity are beyond what is currently technically achievable. Research and development are proceeding to improve assay capability in, for example, direct $\gamma$-ray counting, surface $\alpha$ - and $\beta$-ray counting, and mass spectroscopy.

At such extreme low levels of activity from $\mathrm{U}$ and $\mathrm{Th}$, other components of the continuum that were previously a secondary concern may contribute a significant fraction to the background continuum. In fact, it may be that no single component will dominate and a number of contributors may form a complicated mix. In the remainder of this subsection, we discuss the possible contributors and strategies to mitigate their effects.

Double beta decay experiments are conducted deep underground to avoid cosmic-ray interactions. At these depths, muons are the only surviving cosmic-ray particles, but their interactions can produce high-energy secondaries of neutrons, bremsstralung $\gamma$ rays, and electromagnetic showers. The experimental apparatus itself is usually contained within a detection system for muons. Hence any signal produced by the muon itself or any of its prompt emissions from interactions in the apparatus will be eliminated by an anticoincidence requirement.

Neutrons, being neutral, can have sizable penetrating power through a shield. They generate background through $\left(n, n^{\prime} \gamma\right)$ and $(n, \gamma)$ reactions or $(n, x)$ reactions that lead to radioactive nuclei. In particular, background originating from $\left(n, n^{\prime} \gamma\right)$ reactions within the experimental apparatus produce a rather nondescript spectrum. Any individual level in a nucleus might be excited only weakly, but there are many possible levels. Hence a significant $\gamma$-ray flux without strong identifiable lines can appear, making, neutron-induced backgrounds difficult to diagnose. Neutrons have two sources: fission and $(\alpha, n)$ reactions in the cavity rock where $\mathrm{U}$ and Th levels can be relatively high, and muon interactions in the rock. Fission and $(\alpha, n)$ neutrons have energies less than about $10 \mathrm{MeV}$. These low-energy neutrons can be moderated and shielded from the detector with layers of hydrogenous material. Neutrons arising from muon interactions can have very high energy $(>1 \mathrm{GeV})$ and therefore may penetrate the shield and induce a background-causing reaction near the detector. One particularly dangerous reaction is $\left(n, n^{\prime} \gamma\right)$ in $\mathrm{Pb}$; it can produce $\gamma$ rays with an energy very near to the $Q_{\beta \beta}$ of ${ }^{76} \mathrm{Ge}$ (Mei et al., 2007). Even more onerous is the $\mathrm{Pb}\left(n, n^{\prime} \gamma\right)$-produced $3062-\mathrm{keV} \quad \gamma$ ray, which has a double-escape peak very near $Q_{\beta \beta}$. This single-site energy deposit could be problematic for Ge detector experiments using $\mathrm{Pb}$ as a shield. (See Sec. VII.C.3 for a discussion of the $\mathrm{Ge}$ experiments.) Neutrons from muon-induced reactions can be reduced by going deeper underground.

Fast neutrons, along with other hadrons, can produce radioactive nuclei that may create background for $\beta \beta(0 \nu)$ (Brodzinski et al., 1990; Avignone et al., 1992). For example, ${ }^{68} \mathrm{Ge}$ and ${ }^{60} \mathrm{Co}$ are produced in $\mathrm{Ge}$ and ${ }^{60} \mathrm{Co}$ is also produced in $\mathrm{Cu}$. The production rates are significant on the surface of the Earth where the hadronic cosmic-ray flux is large. Because these nuclei have long half-lives ( 0.8 and $5.3 \mathrm{yr}$, respectively), they will remain in the apparatus and potentially create background after the materials have been placed underground. These backgrounds can be mitigated by storing material underground and letting the isotopes decay away (not practical for $\mathrm{Co}$ ) or by purifying the radioactive isotope from the host material and then minimizing its exposure above ground. Both strategies will be used in future efforts. Anthropogenic isotopes, such as ${ }^{207} \mathrm{Bi}$, have also been observed in $\beta \beta$ experiments (KlapdorKleingrothaus et al., 2004a). Some exotic backgrounds have also been considered. For example, solar neutrinos are unlikely to be a significant background for most experimental configurations (Elliott and Engel, 2004).

\section{B. Facility requirements}

There is no doubt that a laboratory at significant depth is required for $\beta \beta$ experiments. Just how deep is a difficult question to answer, but clearly, deeper is better. However, logistical concerns may induce a collaboration to choose a particular laboratory. The depth requirement for $\beta \beta$ is set by the flux of high-energy, muoninduced neutrons and subsequent reactions that can lead to background (see Sec. VI.A). Depth-requirement calculations are exceedingly difficult to perform. However, two recent works (Mei and Hime 2006; Pandola et al., 2007) have estimated the backgrounds in Ge-detector $\beta \beta(0 \nu)$ experiments. Both found that the background rate at a depth equivalent to that of Gran Sasso [3.1 kmwe flat overburden equivalent (Mei and Hime, 2006)] could be kept below $\sim 1 /$ (tonne yr), but that significant veto efforts would be required. The problematic fast neutron flux decreases with the muon flux, which, in turn drops by a factor of 10 for every $1.5 \mathrm{kmwe}$ in depth. Mei and Hime (2006) estimate that a depth of $5 \mathrm{kmwe}$ (flat overburden) would greatly reduce veto-system requirements.

As the background requirements become more stringent, increasingly heroic efforts will be required to mini- 
mize activity internal to detector materials. In addition, as the experiments get larger and more complicated, the required infrastructure at the experimental site will become more demanding. For example, underground manufacturing of $\mathrm{Cu}$ to avoid cosmogenic activation of ${ }^{60} \mathrm{Co}$, clean room facilities for assembly of sensitive parts, strong exclusion of $\mathrm{Rn}$ to prevent daughters from plating out, and material purification capability are a few of the high-technology requirements for a remote underground location. Since many experiments (in addition to those on $\beta \beta$ ) will need an underground site and similar capabilities, the importance of a major facility to provide common infrastructure is clear. The opportunities and requirements for a deep underground laboratory have been described by Beier et al. (2006).

\section{Measurements to constrain nuclear matrix elements}

Part of the $\beta \beta$ program is an effort to reduce uncertainty in the nuclear-physics factors that, along with $\left\langle m_{\beta \beta}\right\rangle$, determine the rate of $\beta \beta(0 \nu)$. What data will be most useful? Zuber (2005) addressed this question and summarized measurements that might help clear up nuclear-physics issues. Such measurments, should focus on high $Q_{\beta \beta}$ isotopes (the decay rate is proportional to $Q_{\beta \beta}^{5}$, even though low $Q_{\beta \beta}$ isotopes such as ${ }^{128} \mathrm{Te}$ and ${ }^{238} \mathrm{U}$ are of some interest. The 11 isotopes with a $Q_{\beta \beta}$ greater than $2.0 \mathrm{MeV}$ are ${ }^{48} \mathrm{Ca},{ }^{76} \mathrm{Ge},{ }^{82} \mathrm{Se},{ }^{96} \mathrm{Zr},{ }^{100} \mathrm{Mo}$, ${ }^{110} \mathrm{Pd},{ }^{116} \mathrm{Cd},{ }^{124} \mathrm{Sn},{ }^{130} \mathrm{Te},{ }^{136} \mathrm{Xe}$, and ${ }^{150} \mathrm{Nd}$. Most of these isotopes are currently part of a $\beta \beta$ experimental program.

The $Q_{\beta \beta}$ for these nuclei are known to only a few $\mathrm{keV}$, with the exception of that in ${ }^{76} \mathrm{Ge}$, which is known to $\sim 50 \mathrm{meV}$. Precision measurements of the other $Q$ values would be useful because energy resolution is improving. The energy resolutions of the COBRA $(\sim 55 \mathrm{keV})$ and CUORE $(\sim 5 \mathrm{keV})$ experiments are larger than or comparable to the uncertainty in the $Q$ values (Audi et al., 2003; Redshaw et al., 2007) for their chosen isotopes ( $4 \mathrm{keV}$ for ${ }^{116} \mathrm{Cd}$ and $2 \mathrm{keV}$ for ${ }^{130} \mathrm{Te}$ ). It would also be helpful to measure the masses of certain nuclei involved in radiative electron capture-electron capture decay, because that decay rate can be resonantly enhanced if the energy of the $2 P-1 S$ atomic transition is similar to the $Q$ value (Sujkowski and Wycech, 2004). At present the masses of the interesting nuclei are not known precisely enough to determine the degree of enhancement.

Precise $\beta \beta(2 \nu)$ data in all nuclei used for $\beta \beta(0 \nu)$ and single- $\beta$ data for the associated intermediate nuclei, where possible, are also needed. Rates for both processes have been used to fix the parameter $g_{p p}$ that plays a crucial role in QRPA equations (Rodin et al., 2003, 2006; Suhonen, 2005) (see Sec. V.B). More data would test internal consistency, and help decide which $g_{p p}$ calibrator is better.

In $\beta \beta(2 \nu)$, and in $\beta \beta(0 \nu)$ as well when the closure approximation is not used, expressions for the overall matrix element [see Eqs. (38) and (24)] contain matrix elements of the charge changing weak current from both the parent and daughter nuclei to states in the intermediate nucleus. In the long-wavelength approximation, which the high-momentum virtual neutrino prevents for $\beta \beta(0 \nu)$, these reduce to the Fermi and Gamow-Teller (GT) operators. GT strength distributions from the parent nucleus in the relevant isotopes can be measured with charge-exchange reactions (Ejiri, 2000; Amos et al., 2007), and should be measured for all nine high- $Q$-value isotopes of experimental interest. Extracting GT strengths from the daughter (Amos et al., 2007) and the strengths for higher-multipole analogs of the GT operator is critical for $\beta \beta(0 \nu)$ but much harder because the relations between the cross sections and operator matrix elements are more complicated, and polarized beams are sometimes required. Transitions from the parent nucleus can be studied, e.g., with $(p, n)$ or $\left({ }^{3} \mathrm{He}, t\right)$ reactions and transitions from the daughter nucleus with $(n, p),\left(d,{ }^{2} \mathrm{He}\right)$, or $\left(t,{ }^{3} \mathrm{He}\right)$. But even if the connection between cross sections and transition strengths can be made, the strength is still the square of a matrix element, so that the corresponding sign, important for $\beta \beta$, is unavailable.

Muon capture is governed by matrix elements similar to those connecting the intermediate and daughter nuclei in $\beta \beta$. Because the muon is heavy, states with any multipole can be populated during capture, and partial muon capture rates to specific states can be linked to the corresponding parts of the $\beta \beta$ matrix elements (Kortelainen and Suhonen, 2002). Volpe (2005) proposed charged-current neutrino-nucleus scattering as a technique to constrain $M_{0 v}$. By using both $\nu$ and $\bar{\nu}$, one can probe matrix elements of one-body operators to intermediate states from both the parent and daughter nucleus.

The study of pair correlations of nucleons in the ground state of the parent and daughter nuclei allows one to probe aspects of the wave functions that are important for $\beta \beta$ matrix elements even when the closure approximation is used. Double beta decay can be represented as the removal of two neutrons followed by the addition of two protons, and calculations show that when the representation is decomposed into multipoles, the $J^{\pi}=0^{+}$channel is the most important. Recent results of $(p, t)$ reactions for the Ge-Se system (Freeman et al., 2007) have shown no evidence of pairing vibrations that have been found in low- $A$ systems. ${ }^{76} \mathrm{Ge}$ and ${ }^{76} \mathrm{Se}$ have quantitatively similar neutron pairing correlations, and calculations will be easier there than they would be otherwise.

Other processes, such as pion double-charge exchange, electromagnetic transitions to isobaric analog states (Ejiri, 2006a), and $\beta \beta(2 \nu)$ to the excited state in the daughter nucleus (Aunola and Suhonen, 1996; Suhonen and Civitarese, 1998), can also help constrain $\beta \beta(0 \nu)$ matrix elements. But despite the usefulness of these auxiliary measurements, none will provide a magic bullet. Such data will help judge the quality of calcula- 
TABLE II. A list of the values of $T_{1 / 2}^{2 \nu}$ for various isotopes. These values are recommended by Barabash (2006a) as the best interpretation of the experimental data. One should heed the discussion in Barabash (2006a) before using the values in a quantitative way.

\begin{tabular}{lccc}
\hline \hline Isotope & $T_{1 / 2}^{2 \nu}(\mathrm{yr})$ & Isotope & $T_{1 / 2}^{2 \nu}(\mathrm{yr})$ \\
\hline${ }^{48} \mathrm{Ca}$ & $\left(4.2_{-1.0}^{+2.1}\right) \times 10^{19}$ & ${ }^{128} \mathrm{Te}$ & $(2.5 \pm 0.3) \times 10^{24}$ \\
${ }^{76} \mathrm{Ge}$ & $(1.5 \pm 0.1) \times 10^{21}$ & ${ }^{130} \mathrm{Ba} \mathrm{EC}-\mathrm{EC}(2 \nu)$ & $(2.2 \pm 0.5) \times 10^{21}$ \\
${ }^{82} \mathrm{Se}$ & $(0.92 \pm 0.07) \times 10^{20}$ & ${ }^{130} \mathrm{Te}$ & $(0.9 \pm 0.1) \times 10^{21}$ \\
${ }^{96} \mathrm{Zr}$ & $(2.0 \pm 0.3) \times 10^{19}$ & ${ }^{150} \mathrm{Nd}$ & $(7.8 \pm 0.7) \times 10^{18}$ \\
${ }^{100} \mathrm{Mo}$ & $(7.1 \pm 0.4) \times 10^{18}$ & ${ }^{238} \mathrm{U}$ & $(2.0 \pm 0.6) \times 10^{21}$ \\
${ }^{116} \mathrm{Cd}$ & $(3.0 \pm 0.2) \times 10^{19}$ & & \\
\hline \hline
\end{tabular}

tions, but not reduce uncertainty to near zero. For that, the best data are probably $T_{1 / 2}^{0 \nu}$ themselves, the same quantities one is trying to calculate. If the lifetimes are measured in several nuclei, one can check directly whether the calculations work, most simply by fixing $\left\langle m_{\beta \beta}\right\rangle$ from one nucleus and seeing whether the other lifetimes are correctly predicted. Of course, this assumes that light-neutrino exchange rather than some other mechanism is driving the decay.

\section{FUTURE PROGRAM}

\section{A. Previous experiments}

Since the first direct observation of $\beta \beta(2 \nu)$ in 1987 (Elliott et al., 1987), decay rates in a number of other isotopes have been measured, either directly or with geochemical-radiochemical techniques. The work of Rodin et al. $(2003,2006)$ has rekindled interest in the precise measurements of $T_{1 / 2}^{2 \nu}$. Barabash (2006a), in a review of the $\beta \beta(2 \nu)$ experiments, has recommended average $T_{1 / 2}^{2 \nu}$ values, which we quote in Table II.
Here we note that because $\Gamma_{2 \nu}$ is proportional to $Q_{\beta \beta}^{1}$, whereas $\Gamma_{0 \nu}$ is proportional to $Q_{\beta \beta}^{5}, \Gamma_{0 \nu}$ might be larger than $\Gamma_{2 \nu}$ if $Q_{\beta \beta}$ is small and geochemical measurements of the decay rates that do not distinguish between $\beta \beta(0 \nu)$ and $\beta \beta(2 \nu)$ might still produce a competitive limit on $\left\langle m_{\beta \beta}\right\rangle$. This is the case in ${ }^{128} \mathrm{Te}$, where the $T_{1 / 2}^{0 \nu}$ given in Table II produces a limit on $\left\langle m_{\beta \beta}\right\rangle$ (Bernatowicz et al., 1993) that is only slightly worse than the best limits given in Table III. The nucleus ${ }^{238} \mathrm{U}$ is another case (Turkevich et al., 1991) in which the radiochemical experiment could not distinguish $\beta \beta(0 \nu)$ and $\beta \beta(2 \nu) .{ }^{328} \mathrm{U}$ decays to ${ }^{238} \mathrm{Pu}$, which in turn $\alpha$ decays with a $87.7 \mathrm{yr}$ half-life. A sample of $\mathrm{U}$ salt had been stored for $33 \mathrm{yr}$ and was milked for its Pu content. By counting the ${ }^{238} \mathrm{Pu}$ $\alpha$, the $\beta \beta$ half-life was determined. Because of its low $Q_{\beta \beta}$ the high observed decay rate (compared to the theoretically predicted $T_{1 / 2}^{2 \nu}$ ) has been interpreted as evidence for $\beta \beta(0 \nu)$. Unfortunately, the experiment is difficult to repeat because of the special nature of the U-salt sample available to the experimenters.

The entire history of $\beta \beta$ measurements up to about 2001 , including $\beta \beta(2 \nu), \beta \beta(0 \nu)$, and Majoron modes, can be found in Tretyak and Zdesenko (2002). The best experiments to date have been in Ge. The HeidelbergMoscow (Klapdor-Kleingrothaus et al., 2001a) and IGEX (Aalseth et al., 2002a, 2004) experiments have provided the best limits on $T_{1 / 2}^{0 \nu}$ by building detectors with the lowest backgrounds.

\section{B. Overview of the future program}

In the inverted hierarchy with a $m_{\text {lightest }}$ near $0 \mathrm{meV}$, $\left\langle m_{\beta \beta}\right\rangle$ will be near $20-50 \mathrm{meV}$. The $T_{1 / 2}^{0 \nu}$ resulting from this neutrino mass will be near $10^{27} \mathrm{yr}$ with a resulting count rate of few/(tonne yr). On the other hand, if the recent claim of $\left\langle m_{\beta \beta}\right\rangle \sim 400 \mathrm{meV}$ is borne out, the halflife will be nearer to $10^{25} \mathrm{yr}$, with a count rate of a few $100 /$ (tonne yr). A precision measurement $(20 \%$ or bet-

TABLE III. A list of recent $\beta \beta(0 \nu)$ experiments and their $90 \%$ confidence level (except as noted) limits on $T_{1 / 2}^{0 \nu}$. The $\left\langle m_{\beta \beta}\right\rangle$ limits, if provided, are those quoted by the authors, who each made choices about which calculated $M_{0 \nu}$ to use.

\begin{tabular}{|c|c|c|c|c|}
\hline Isotope & Technique & $T_{1 / 2}^{0 \nu}$ & $\left\langle m_{\beta \beta}\right\rangle(\mathrm{eV})$ & Reference \\
\hline${ }^{48} \mathrm{Ca}$ & $\mathrm{CaF}_{2}$ scint. crystals & $>1.4 \times 10^{22} \mathrm{yr}$ & $<7.2-44.7$ & Ogawa et al. (2004) \\
\hline${ }^{76} \mathrm{Ge}$ & ${ }^{\text {enr }} \mathrm{Ge}$ det. & $>1.9 \times 10^{25} \mathrm{yr}$ & $<0.35$ & Klapdor-Kleingrothaus et al. (2001a) \\
\hline${ }^{76} \mathrm{Ge}$ & ${ }^{\text {enr }} \mathrm{Ge}$ det. & $\left(2.23_{-0.31}^{+0.44}\right) \times 10^{25} \mathrm{yr}(1 \sigma)$ & $0.32 \pm 0.03$ & Klapdor-Kleingrothaus and Krivosheina (2006) \\
\hline${ }^{76} \mathrm{Ge}$ & ${ }^{\mathrm{enr}} \mathrm{Ge}$ det. & $>1.57 \times 10^{25} \mathrm{yr}$ & $<0.33-1.35$ & Aalseth et al. (2002a) \\
\hline${ }^{82} \mathrm{Se}$ & Thin metal foils and tracking & $>2.1 \times 10^{23} \mathrm{yr}$ & $<1.2-3.2$ & Barabash (2006b) \\
\hline${ }^{100} \mathrm{Mo}$ & Thin metal foils and tracking & $>5.8 \times 10^{23} \mathrm{yr}$ & $<0.6-2.7$ & Barabash (2006b) \\
\hline${ }^{116} \mathrm{Cd}$ & ${ }^{116} \mathrm{CdWO}_{4}$ scint. crystals & $>1.7 \times 10^{23} \mathrm{yr}$ & $<1.7$ & Danevich et al. (2003) \\
\hline${ }^{128} \mathrm{Te}$ & Geochemical & $>7.7 \times 10^{24} \mathrm{yr}$ & $<1.1-1.5$ & Bernatowicz et al. (1993) \\
\hline${ }^{130} \mathrm{Te}$ & $\mathrm{TeO}_{2}$ bolometers & $>3.0 \times 10^{24} \mathrm{yr}$ & $<0.41-0.98$ & Arnaboldi et al. (2007) \\
\hline${ }^{136} \mathrm{Xe}$ & Liquid Xe scint. & $>4.5 \times 10^{23} \mathrm{yr}^{\mathrm{a}}$ & $<0.8-5.6$ & Bernabei et al. (2002) \\
\hline${ }^{150} \mathrm{Ne}$ & Thin metal foils and tracking & $>3.6 \times 10^{21} \mathrm{yr}$ & & Barabash (2005) \\
\hline
\end{tabular}

${ }^{\mathrm{a}}$ See footnote 4 in Bernabei et al. (2002). 
ter) requires a few hundred events. To make such a measurement in a 2-3-yr run, it seems prudent to build an experiment containing $100-200 \mathrm{~kg}$ of isotope that can be expanded to 1 ton at a later time. But collecting statistics in a reduced background experiment is not the only goal. The recent claim for $\beta \beta(0 \nu)$ is controversial. Future claims must provide supporting evidence to strengthen the argument (Elliott, 2006). This evidence might include any number of the following:

- To show that $\beta \beta(0 \nu)$ likely exists, one needs a combination of

(i) a clear peak at the correct $\beta \beta(0 \nu)$ energy,

(ii) a demonstration that the event is a single-site energy deposit,

(iii) measured event distributions (spatial, temporal) representative of $\beta \beta(0 \nu)$,

(iv) a demonstration that the measured decay rate scales with isotope fraction.

- To present a convincing case, one needs

(i) an observation of the two-electron nature of the $\beta \beta(0 \nu)$ event,

(ii) a demonstration that the kinematic distributions (electron energy sharing, opening angle) match those of $\beta \beta(0 \nu)$,

(iii) to observe the daughter nucleus together in time with the $\beta \beta(0 \nu)$ decay,

(iv) to observe excited-state decay with parameters indicating $\beta \beta(0 \nu)$.

- To remove all doubt, many of the above $\beta \beta(0 \nu)$ indicators should be measured in several isotopes.

The proposals summarized below in Sec. VII.C address these possibilities in different ways.

\section{The number of required experiments and their precision}

If $\beta \beta(0 \nu)$ is observed, the qualitative physics conclusion that neutrinos have a Majorana nature will have a profound effect on the development of models for mass generation. Quantifying the physics associated with lepton-number violation will be more challenging because of the uncertainty in $M_{0 v}$. If the matrix elements were precisely known, one could compare $\Gamma_{0 \nu}$ measurements in several nuclei to address the question of the underlying mechanism. If, in addition, the mechanism was certain, one could determine the LNVP from a lone $\Gamma_{0 \nu}$ measurement.

Recently a number of papers have appeared (Bahcall et al., 2004a; Bilenky et al., 2004; Deppisch and Päs, 2007; Gehman and Elliott, 2007) that try to quantify, in light of matrix-element uncertainties, the number and type of experimental results needed to draw quantitative conclusions. Although the papers make different assumptions, the concensus is that if $\beta \beta(0 \nu)$ exists, measurements of $\Gamma_{0 \nu}$ in at least three different isotopes are

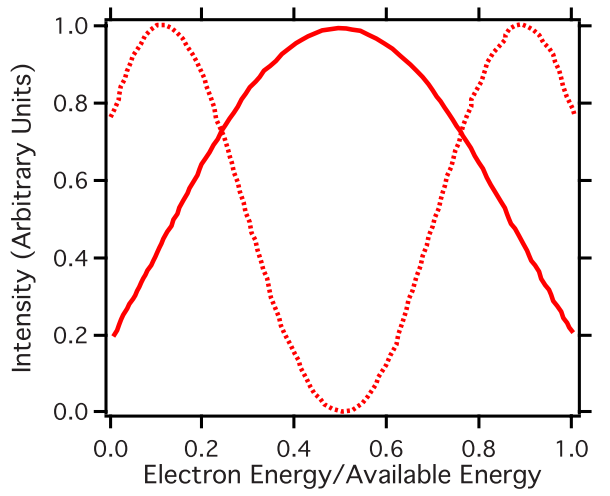

FIG. 6. (Color online) Distribution of the electron energies taken one at a time for $\beta \beta(0 \nu)$. The solid curve is for lightneutrino exchange and the dotted curve is for right-handed currents in both the leptonic and hadronic currents (Doi et al., 1985).

warranted. All the analyses assume that existing calculations provide an estimate of the difference in rates produced by different mechanisms, and require that the experiments determine the rate to within $20 \%$. This last number comes from the level of variation in calculated matrix elements.

\section{Kinematic distributions}

If $\beta \beta(0 \nu)$ is observed, future experimenters will want to study the kinematic distributions associated with the outgoing electrons. The NEMO 3 experiment (Arnold et al., 2005a) has shown the power of this type of analysis with $\beta \beta(2 \nu)$. The collaboration used the spectrum of electron energies from $\beta \beta(2 \nu)$ in ${ }^{100} \mathrm{Mo}$, taken one at a time (the lone electron spectrum), to show that the decay proceeds predominately through the $1^{+}$ground state of the intermediate ${ }^{100} \mathrm{Tc}$ nucleus. The effect on the spectrum of this single-state dominance is small (Šimkovic et al., 2001), so $10^{5}$ events were required for the analysis. But the analysis demonstrates that important information is contained within kinematic distributions.

Figure 6 shows an example of the kinematic distributions for $\beta \beta(0 \nu)$. In this figure, the lone electron spectra are shown for two possible exchange mechanisms. The mountain shaped curve is for light-neutrino exchange mechanism and the valley shaped curve is for righthanded interactions in both the leptonic and hadronic currents (Doi et al., 1985). It is clear from this figure that only a few tens of events would be needed to show that one of these mechanisms dominates the decay. However, a large sample of events would permit an analysis to constrain the fraction of a competing mechanism. The opening-angle distribution is another observable that is sensitive to aspects of the underlying exchange mechanism.

The only detectors able to register these types of distributions involve thin-foil, tracking-volume sandwiches. The MOON (Nakamura et al., 2007) and SuperNEMO (Barabash, 2004) proposals are such examples. There are 
TABLE IV. A summary list of the $\beta \beta(0 \nu)$ proposals and experiments.

\begin{tabular}{|c|c|c|c|c|c|}
\hline Experiment & Isotope & Mass & Technique & Present status & Reference \\
\hline CANDLES & ${ }^{48} \mathrm{Ca}$ & few tons & $\mathrm{CaF}_{2}$ scint. crystals & Prototype & Umehara et al. (2006) \\
\hline CARVEL & ${ }^{48} \mathrm{Ca}$ & 1 ton & $\mathrm{CaWO}_{4}$ scint. crystals & Development & Zdesenko et al. (2005) \\
\hline COBRA & ${ }^{116} \mathrm{Cd}$ & $418 \mathrm{~kg}$ & CZT semicond. det. & Prototype & Zuber (2001) \\
\hline CUORICINO & ${ }^{130} \mathrm{Te}$ & $40.7 \mathrm{~kg}$ & $\mathrm{TeO}_{2}$ bolometers & Running & Arnaboldi et al. (2005) \\
\hline CUORE & ${ }^{130} \mathrm{Te}$ & $741 \mathrm{~kg}$ & $\mathrm{TeO}_{2}$ bolometers & Proposal & Ardito et al. (2005) \\
\hline DCBA & ${ }^{150} \mathrm{Ne}$ & $20 \mathrm{~kg}$ & ${ }^{\text {enr }} \mathrm{Nd}$ foils and tracking & Development & Ishihara et al. (2000) \\
\hline EXO-200 & ${ }^{136} \mathrm{Xe}$ & $200 \mathrm{~kg}$ & Liq. ${ }^{e n r} \mathrm{Xe} \mathrm{TPC} /$ scint. & Construction & Piepke (2007) \\
\hline EXO & ${ }^{136} \mathrm{Xe}$ & $1-10$ ton & Liq. ${ }^{e n r} \mathrm{Xe}$ TPC/scint. & Proposal & Danilov et al. (2000) \\
\hline GEM & ${ }^{76} \mathrm{Ge}$ & 1 ton & ${ }^{\mathrm{enr}} \mathrm{Ge}$ det. in liq. nitrogen & Inactive & Zdesenko et al. (2001) \\
\hline GENIUS & ${ }^{76} \mathrm{Ge}$ & 1 ton & ${ }^{\mathrm{enr}} \mathrm{Ge}$ det. in liq. nitrogen & Inactive & Klapdor-Kleingrothaus et al. (2001a) \\
\hline GERDA & ${ }^{76} \mathrm{Ge}$ & $\approx 35 \mathrm{~kg}$ & ${ }^{\mathrm{enr}} \mathrm{Ge}$ semicond. det. & Construction & Schönert et al. (2005) \\
\hline GSO & ${ }^{160} \mathrm{Gd}$ & 2 ton & $\mathrm{Gd}_{2} \mathrm{SiO}_{5}: \mathrm{Ce}$ crys. scint. in liq. scint. & Development & Danevich et al. (2000); Wang et al. (2002) \\
\hline MAJORANA & ${ }^{76} \mathrm{Ge}$ & $120 \mathrm{~kg}$ & ${ }^{\mathrm{enr}} \mathrm{Ge}$ semicond. det. & Proposal & Gaitskell et al. (2003) \\
\hline MOON & ${ }^{100} \mathrm{Mo}$ & 1 ton & enr Mo foils/scint. & Proposal & Nakamura et al. (2007) \\
\hline $\mathrm{SNO}++$ & ${ }^{150} \mathrm{Nd}$ & 10 ton & Nd loaded liq. scint. & Proposal & Chen (2005) \\
\hline SuperNEMO & ${ }^{82} \mathrm{Se}$ & $100 \mathrm{~kg}$ & ${ }^{\mathrm{enr}} \mathrm{Se}$ foils/tracking & Proposal & Barabash (2004) \\
\hline $\mathrm{Xe}$ & ${ }^{136} \mathrm{Xe}$ & 1.56 ton & ${ }^{\mathrm{enr}} \mathrm{Xe}$ in liq. scint. & Development & Caccianiga and Giammarchi (2001) \\
\hline XMASS & ${ }^{136} \mathrm{Xe}$ & 10 ton & Liquid Xe & Prototype & Takeuchi (2008) \\
\hline HPXe & ${ }^{136} \mathrm{Xe}$ & tons & High pressure Xe gas & Development & Nygren (2007) \\
\hline
\end{tabular}

competing demands in the design of such experiments. One would like a thick foil for a large source mass, but a thin foil to minimize energy loss and scattering so the integrity of the distributions will be retained. As a result, such experiments will have to be large with many electronic channels in order to collect the required numbers of events.

\section{The experiments}

In this section, we describe a number of proposed experimental programs for $\beta \beta(0 \nu)$. A summary of the proposals is given in Table IV.

\section{CUORE (Cryogenic Underground Observatory for Rare Events)}

Before addressing the CUORE experiment, it is appropriate to discuss the general principles of cryogenic, or thermal, detectors, and the pilot experiment, CUORICINO. The detector is an array of bolometers made from a material containing the parent decay isotope. In CUORICINO and CUORE the individual bolometers are $5 \times 5 \times 5 \mathrm{~cm}^{3}$ single crystals of $\mathrm{TeO}_{2}$. They are made from natural-abundance Te, which is $33.8 \%$ ${ }^{130} \mathrm{Te}$. They are operated at very low temperatures where they have tiny specific heats. They are weakly thermally coupled to copper support frames that act as the heat bath. When an event leaves energy in the crystal, the temperature rises and that rise is measured by a sensitive thermistor, which produces a signal proportional to the energy deposited. The heat leaks back through the weak thermal couplings and the signal decays as the temperature returns to its baseline.

This technique was suggested for $\beta \beta$ decay searches by Fiorini and Niinikoski (1984), and applied earlier by the Milano group in the MIBETA experiment (Arnaboldi et al., 2003) before CUORICINO (Arnaboldi et al., 2005). The CUORICINO bolometers are dielectric and diamagnetic, and are operated at temperatures between 8 and $10 \mathrm{mK}$ (Arnaboldi et al., 2003, 2004, 2005). According to the Debye law, the specific heat of $\mathrm{TeO}_{2}$ crystals is given by $C(T)=\beta\left(T / \Theta_{D}\right)^{3}$, where $\beta$ $=1994 \mathrm{~J} /(\mathrm{K} \mathrm{mol})$ and $\Theta_{D}$ is the Debye temperature. In these materials, $C(T)$ is due almost exclusively to lattice degrees of freedom. The constant $\Theta_{D}$ was measured by the Milan group for $5 \times 5 \times 5 \mathrm{~cm}^{3} \mathrm{TeO}_{2}$ crystals as $232 \mathrm{~K}$ (Arnaboldi et al., 2003), which differs from the previously published value of $272 \mathrm{~K}$ (White et al., 1990). The specific heat follows the Debye law down to $60 \mathrm{mK}$. The heat capacity of the crystals, extrapolated to $10 \mathrm{mK}$, is $2.3 \times 10^{-9} \mathrm{~J} / \mathrm{K}$. With these values of the parameters, an energy deposition of a few $\mathrm{keV}$ will result in a measurable temperature increase $\Delta T$. The temperature increase caused by the deposition of energy equal to the total $\beta \beta(0 \nu)$ decay energy, $Q_{\beta \beta}=2530 \mathrm{keV}$, would be $0.177 \mathrm{mK}$. To obtain usable signals for such small temperature changes, very sensitive thermistors are required. In CUORICINO, $\Delta T$ is measured by high resistance germanium thermisters glued to each crystal. More details can be found in Fiorini and Niinikoski (1984), and in subsequent publications (Allessandrello et al., 1997, 1998). 
The thermistors are heavily doped high-resistance germanium semiconductors, with an impurity concentration slightly below the metal-insulator transition. Highquality thermistors require a very homogeneous doping concentration. For the CUORICINO thermistors [neutron transmutation doped (NTD)], this was achieved by uniform thermal neutron irradiation throughout the entire volume in a nuclear reactor. The electrical conductivity of the devices depends very sensitively on the temperature because they use variable range hopping (VRH) mechanisms. The resistivity varies with temperature according to $\rho=\rho_{0} \exp \left(T_{0} / T\right)^{\gamma}$, where the constants $\rho_{0}, T_{0}$, and $\gamma$ all depend on the doping concentration. In the case of thermistors operating with VRH mechanisms, $\gamma=1 / 2$.

Thermistors can be characterized by their sensitivity $A(T)$ defined as follows: $A(T)=|d(\ln R) / d(\ln T)|$ $=\gamma\left(T_{0} / T\right)^{\gamma}$, where the resistance is $R(T)$ $=R_{0} \exp \left(T_{0} / T\right)^{\gamma}$. The parameter $R_{0}=\rho_{0}(d / a)$, where $d$ and $a$ are the distance between the contacts and the cross section of the thermistor, respectively. The values of $R_{0}, T_{0}$, and $\gamma$ must be measured for each thermistor. This is done by coupling the thermistor to a lowtemperature heat sink with a high heat-conductivity epoxy. The base temperature of the heat sink is between 15 and $50 \mathrm{mK}$. A current flows through the device and a $V$ - $I$ load curve is plotted. The curve becomes nonlinear because of the power dissipation and dynamic resistance, causing the slope of the $I(V)$ curve to change from positive to negative. The optimum operating biasing current occurs where $d I / d V$ increases rapidly with increasing bias voltage $V_{b}$, maximizing the signal to noise ratio. The parameters of each thermister are determined from a combined fit to a set of load curves at different base temperatures. The characterization process was described in detail earlier for $\mathrm{Si}$ thermistors (Allessandrello et al., 1999), and the same process is used for the CUORICINO Ge thermistors.

The thermistors in CUORE will be the same as those used in CUORICINO; they were produced by the UC Berkeley-LBNL group (Haller et al., 1982). It is necessary to optimize the neutron doping of the Ge. This is done by using foils of metal with long-lived $(n, \gamma)$ radioactive daughter nuclides irradiated along with the Ge to calibrate the neutron flux. Accordingly the neutron exposure can be accurately evaluated without having to wait for the intense radiation of the ${ }^{71} \mathrm{Ge}$ in the sample to decay. Following the decay period, the Ge is heat treated to repair the crystal structure, and cut into 3 $\times 3 \times 1-\mathrm{mm}^{3}$ strips. Electrical connections are made by two $50-\mu \mathrm{m}$ gold wires, ball bonded to metalized surfaces on the thermistor. The thermistors are glued to each bolometer with nine spots of epoxy, deposited by an array of pins.

Figure 7 shows the CUORICINO structure. Each of the upper ten planes and the lowest one consists of four $5 \times 5 \times 5-\mathrm{cm}^{3}$ crystals of natural isotopic abundance, as shown in the upper right hand figure, while the 11th and 12th planes have $93 \times 3 \times 6-\mathrm{cm}^{3}$ crystals, as shown in the

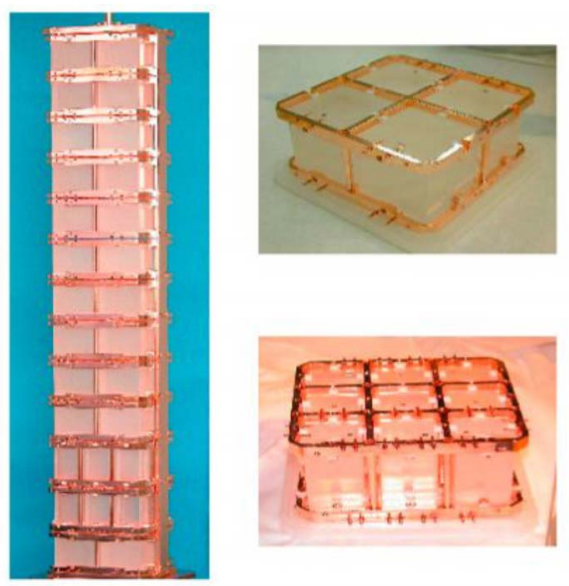

FIG. 7. (Color online) The CUORICINO structure. Figure courtesy of the CUORE Collaboration.

lower right hand figure. In the $3 \times 3 \times 6-\mathrm{cm}^{3}$ planes the central crystal is fully surrounded by the others.

The crystals for CUORE will be prepared the same way as for CUORICINO. The crystals were grown with pre-tested low-radioactivity material by the Shanghai Institute of Ceramics of the Academy of Science (SICAS) and shipped to Italy by sea to minimize activation by cosmic rays. They were lapped with a specially selected low contamination polishing compound. All these operations, as well as the mounting of the tower, were carried out in a nitrogen atmosphere glove box in a clean room. The mechanical structure is made of OFHC copper and Teflon, both of which were previously tested to be sure that measurable radioactive contaminations were minimal. Thermal pulses are recorded by the NTD Ge thermistors thermally coupled to each crystal. The gain of each bolometer is calibrated and stabilized by a $50-100-\mathrm{k} \Omega$ resistor attached to each absorber and acting as a heater. Heat pulses are periodically supplied by a calibrated pulser (Arnaboldi et al., 2004). The tower is suspended from the $50-\mathrm{mK}$ plate of the cryostat through a 25-mm copper bar attached to a steel spring. The steel spring provides mechanical isolation from vibration of the cryostat that can result in heating and spurious pulses in the detector.

The CUORE detector will be an array of 19 towers, each similar to the CUORICINO tower except that all crystals will be $5 \times 5 \times 5 \mathrm{~cm}^{3}$ (see Fig. 8 ). It will have 988 $760 \mathrm{~g}$ bolometers, containing a total of about $750 \mathrm{~kg}$ of $\mathrm{TeO}_{2}$, or $\approx 200 \mathrm{~kg}$ of ${ }^{130} \mathrm{Te}$. The expected background is projected from the CUORICINO background, and the improvements thus far achieved by the collaboration, as well as the effect of coincidence cancellation due to the granularity of the detector. The predicted background rate for the first phase of the experiment is 0.01 counts $/(\mathrm{keV} \mathrm{kg} \mathrm{yr})$. Substituting into Eq. (5) with $n_{\sigma}=1, a=0.348, \epsilon=0.84, W=162, M=760 \mathrm{~kg}, t=10 \mathrm{yr}$, $\delta(E)=7 \mathrm{keV}$, and $b=0.01$ counts $/(\mathrm{keV} \mathrm{kg} \mathrm{yr})$ the predicted sensitivity is $T_{1 / 2}^{0 \nu}\left({ }^{130} \mathrm{Te}\right) \approx 2.5 \times 10^{26} \mathrm{yr}$. According to three recent nuclear structure calculations, $F_{N}$ 


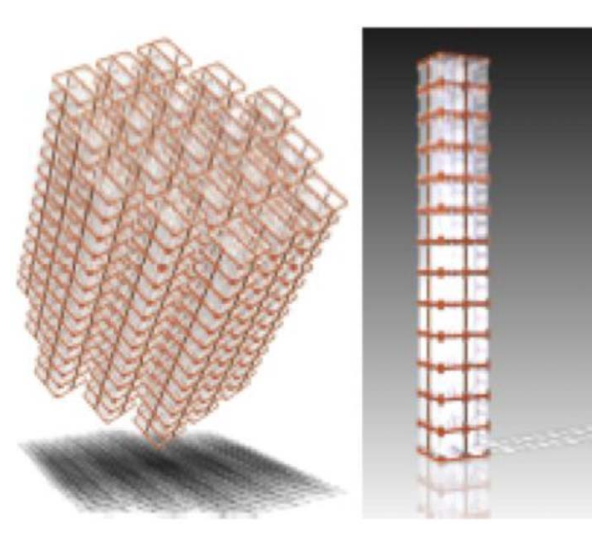

FIG. 8. (Color online) The CUORE structure. Figure courtesy of the CUORE Collaboration.

$=\left(4.84_{-0.64}^{+1.30}\right) \times 10^{-13} / \mathrm{yr} \quad($ Rodin et al., 2006), 2.57 $\times 10^{-13} / \mathrm{yr}$ (Poves et al., 2007), or $5.13 \times 10^{-13} / \mathrm{yr}$ (Civitarese and Suhonen, 2003a, 2003b). These results and the sensitivity above yield $\left\langle m_{\beta \beta}\right\rangle$ limits of 47,53 , and $45 \mathrm{meV}$, respectively. A version of CUORE that is isotopically enriched to $80 \%$ in ${ }^{130} \mathrm{Te}$ would reduce these values by a factor of 1.54 to 31,34 , and $29 \mathrm{meV}$, respectively, which covers the inverted hierarchy if $m_{\text {lightest }}$ is $0 \mathrm{meV}$. The aim of the CUORE Collaboration is to reduce the background further.

The CUORE experiment is approved and funded by the Instituto Nazionale de Fisica Nucleare (INFN) in Italy, and was recently approved for construction and put in the 2008 fiscal-year budget of the U.S. Department of Energy. However, construction has already begun in Laboratori Nazionali del Gran Sasso (LNGS) in Assergi, Italy with Italian funds. Data acquisition is scheduled to begin in 2011. A detailed description has been given by Ardito et al. (2005). The recent results from CUORICINO with a total exposure of $11.8 \mathrm{~kg} \mathrm{yr}$ of ${ }^{130} \mathrm{Te}$ provide a lower limit of $T_{1 / 2}^{0 \nu} \geqslant 3.0 \times 10^{24} \mathrm{yr}$ (Arnaboldi et al., 2007). Using the nuclear structure factors listed above, the bounds on $\left\langle m_{\beta \beta}\right\rangle$ are $0.42,0.58$, and $0.40 \mathrm{eV}$, respectively. The mission of the first phase of CUORE, then, is to improve the half-life sensitivity by a factor of 100 , and that of $\left\langle m_{\beta \beta}\right\rangle$ by one order of magnitude.

\section{EXO (Enriched Xenon Observatory)}

EXO is a proposed large next generation experiment that will search for the $\beta \beta(0 \nu)$ in ${ }^{136} \mathrm{Xe}$. The goal is to use between one and ten tons of Xe enriched to $80 \%$ in ${ }^{136} \mathrm{Xe}$ in a tracking time-projection chamber (TPC). The intent of the collaboration is to trap and identify the daughter ${ }^{136} \mathrm{Ba}$ ion by laser spectroscopy. The decay results in ${ }^{136} \mathrm{Ba}^{++}$ion that will rapidly capture an electron, leading to the ${ }^{136} \mathrm{Ba}^{+}$ion which is stable in $\mathrm{Xe}$. These ions can be positively identified via their atomic spectroscopy by optical pumping with blue and red lasers. This technique was suggested by Moe (1991) and is described in the context of this experiment by Danilov et

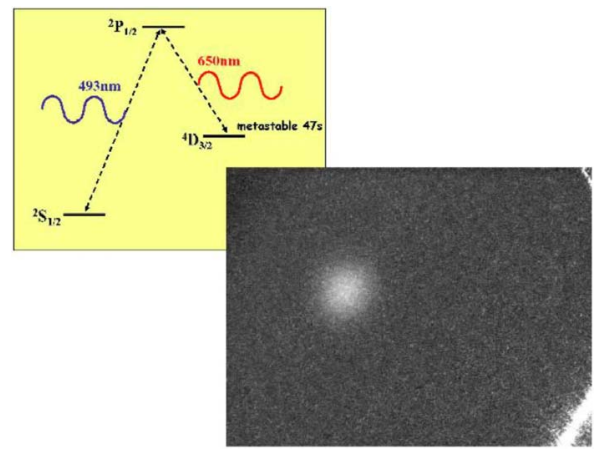

FIG. 9. (Color online) The atomic level structure of a Ba ion indicating the levels of interest and a picture of the emission of a lone ion within an ion trap. Figure courtesy of the EXO Collaboration.

al. (Danilov, 2000). To exploit this method of positively identifying the daughter nucleus, it will be necessary to capture the ion at the decay site and transfer it to an ion trap for optical pumping. Alternatively the ion could possibly be identified at the decay site by directing the lasers. If either of these techniques works, backgrounds that do not result in the correct ion will be eliminated. The development of these techniques is a significant research and development challenge, but one with a very high potential payoff.

The atomic level structure of the $\mathrm{Ba}$ ion is shown in Fig. 9. The 493-nm ground-state transition is very strong allowing the ions in the $6^{2} S_{1 / 2}$ ground state to be optically excited to the $6^{2} P_{1 / 2}$ excited state. The decay of this state has a $30 \%$ branching ratio to the $5^{4} D_{3 / 2}$ metastable state. The $\mathrm{Ba}^{+}$ion is identified by irradiating this $6^{2} P_{1 / 2}$ state with $650-\mathrm{nm}$ red laser light, exciting the ion back to the $6^{2} P_{1 / 2}$ state, which decays with a $70 \%$ branching ratio to the ground state by emitting 493-nm blue light. The $6^{2} P_{1 / 2}$ excited state has a mean life of $8 \mathrm{~ns}$ and when saturated can emit $\sim 10^{7} 493-\mathrm{nm}$ photons per second. This wavelength is compatible with the maximum quantum efficiency of bialkali photomultiplier tubes. The bright glowing spot shown in Fig. 9 is a photograph of an actual excitation in a test trap.

The isotopic enrichment of tons of xenon is technically feasible because xenon is a noble gas that can be directly introduced into a mass-separating centrifuge with no chemistry. In addition, the residual xenon can be returned for normal industrial use. This represents a significant cost saving in the acquisition of the raw xenon. The xenon gas can also be continuously purified during the operation of the experiment, in particular to eliminate the ubiquitous radioactive radon and krypton isotopes.

There are two activities that will presumably lead to the final construction of the ton-level EXO with bariumion tagging. One is the development of the tagging procedure discussed above, and the other is the development of a high-resolution liquid xenon TPC with good tracking ability. The EXO Collaboration has approval and funding to construct a 200-kg liquid TPC with Xe 


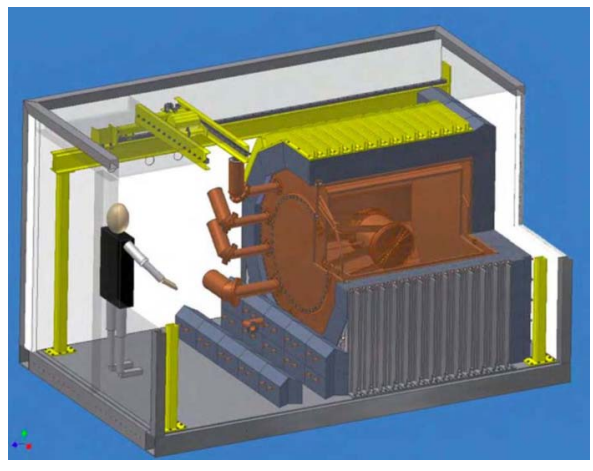

FIG. 10. (Color online) An artist's conception of the EXO-200 experiment. Figure courtesy of the EXO Collaboration.

enriched to $80 \%$ in ${ }^{136} \mathrm{Xe}$. This project is called EXO200. It will not have the barium-ion tagging feature; however, it is intended as a research and development $(\mathrm{R} \& \mathrm{D})$ program for the TPC itself. The enriched isotope has been purchased and is in house. This construction of EXO-200 is presently underway, and the experiment will be installed and operated in the Waste Isolation Pilot Project (WIPP) site in Carlsbad, New Mexico. This site has an overburden of 1600 mwe to reduce the backgrounds associated with cosmic rays. The detector will have an estimated $115 \mathrm{~kg}$ of fiducial volume contained in a thin-walled copper vessel $40 \mathrm{~cm}$ in length. The copper was supplied by a German company, and has been assayed by inductively coupled plasma mass spectroscopy (ICPMS). It contains the following limiting levels of radioactivity: ${ }^{40} \mathrm{~K} \leqslant 6 \mathrm{ppm}, \quad{ }^{232} \mathrm{Th} \leqslant 0.5 \mathrm{ppt}$, and ${ }^{238} \mathrm{U}$ $\leqslant 0.3$ ppt. The copper for the TPC was stored at the Deutches Electron Synchrotron Laboratory (DESY) in Hamburg, Germany, in a concrete bunker to reduce activation from energetic neutrons produced by cosmicray muons. It has now been shipped to Stanford University. It is possible that in the future the collaboration will use Teflon for the vessel; however, the first vessel will be made from this copper. An artist's conception of the EXO-200 is shown in Fig. 10.

The high voltage cathode will be located in the middle of the TPC volume. The design calls for up to $3.5 \mathrm{~V} / \mathrm{cm}$. At each end there are $114 x$ wires and $114 y$ wires (actually at $60^{\circ}$ pitch with respect to the $x$ wires). These are for charge collection, energy, and position information necessary for the reconstruction of the trajectories of the electrons. A 1-MeV $\beta$ particle creates about 50000 electrons in the liquid xenon. One interesting property of xenon gas and liquid is that they are efficient scintillators. The scintillation light will be detected by 258 large area photodiodes on each side of the TPC. The use of both collected charge and scintillator light improves the energy resolution. The combination was tested in a small chamber, and the data imply that EXO-200 will have an energy resolution of about $1.5 \%$.

The installation in the WIPP site is scheduled to occur in mid-2007. The cryostat has been constructed and tested at Stanford University. The EXO Collaboration anticipates reaching a sensitivity of about $0.3 \mathrm{eV}$ for $\left\langle m_{\beta \beta}\right\rangle$. The actual design of the full-size EXO detector will occur after the research and development of the barium-tagging scheme are completed.

\section{Next generation ${ }^{76} \mathrm{Ge}$ double beta decay experiments: MAJORANA and GERDA}

As stated earlier, the most sensitive $\beta \beta(0 \nu)$ experiments so far are the Heidelberg-Moscow (KlapdorKleingrothaus et al., 2001a) and IGEX (Aalseth et al., 2002a, 2004) experiments. In addition, the observation discussed earlier was based on a reanalysis of the Heidelberg-Moscow data (Klapdor-Kleingrothaus et al., 2001a, 2003, 2004a, 2004b; Klapdor-Kleingrothaus and Krivosheina, 2006). The goals of the MAJORANA and GERDA ${ }^{76} \mathrm{Ge}$ experiments are twofold, first to confirm or refute the claimed evidence, and second, in the case of a positive result, to make a measurement of the halflife with a $20 \%$ or better uncertainty. If the claim is not correct, the goal is to make the most sensitive search for this decay. We discuss the MAJORANA experiment first because it will use improved ultralow background cryostat technology similar to that used in the IGEX experiment, which, except for the copper, was similar to that used in the Heidelberg-Moscow experiment. By contrast, the GERDA Collaboration is pursuing a novel technique of cooling and shielding the bare Ge detectors by directly immersing them in liquid argon (Heusser, $1995)$ and water as low- $Z$ shielding. This technique was the cornerstone of a proposal by the GENIUS (Germanium Nitrogen Underground Setup) Collaboration (Klapdor-Kleingrothaus, 1997; Klapdor-Kleingrothaus et al., 2001b) and was tested in an initial experiment at Gran Sasso in the GENIUS test facility (KlapdorKleingrothaus et al., 2003). Similarly the GEM (Germanium Experiment for Neutrino Mass) proposal (Zdesenko et al., 2001) also involved bare Ge detectors, but with pure water forming the shield instead of liquid nitrogen. Neither the GEM nor GENIUS Collaboration is currently active. In the end, the MAJORANA and GERDA Collaborations envision a joint 1-ton experiment, using whichever of their two experimental techniques proves to be the most appropriate to reach the final goals.

${ }^{76}$ Ge decay has several significant advantages. First, all three recent nuclear structure calculations are in fair agreement and predict large nuclear structure factors $F_{N}$. They are $F_{N}=1.22_{-0.11}^{+0.10} \times 10^{-14} \mathrm{yr}$ (Rodin et al., 2003, 2006), $4.29 \times 10^{-14} \mathrm{yr}$ (Poves et al., 2007), and 7.01 $\times 10^{-14} \mathrm{yr}$ (Civitarese and Suhonen, 2003a, 2003b). $F_{N}$ is proportional to the square of the nuclear matrix element, so the matrix element differences themselves are not large, whereas in previous estimates they varied by a factor of 3 . It is generally believed that more reliable shell model calculations will be possible in the future for this nucleus. In addition, ${ }^{76} \mathrm{Ge}$ experiments have the lowest background demonstrated thus far. The background rates at IGEX and Heidelberg-Moscow were $\approx 0.06 \mathrm{count} /(\mathrm{keV} \mathrm{kg} \mathrm{yr})$, after the application of pulse- 
shape analysis. Many of the backgrounds have been identified, and both future experiments are projecting significant reductions. Finally, Ge detectors have the best energy resolution of any of the proposed experimental techniques for double beta decay experiments. Resolutions of $3 \mathrm{keV}$ at the decay energy of $2039 \mathrm{keV}$, or $\sim 0.15 \%$ full width at half maximum, have been achieved. The main difference between the MAJORANA and GERDA proposals are the method of cooling the detectors, and shielding and background control. Both propose to use detector segmentation and pulse-shape discrimination to reduce backgrounds. The goals of both proposed experiments is to probe the quasidegenerate neutrino mass region above $100 \mathrm{meV}$ region in the initial phases. In a subsequent phase, and possibly with collaboration between MAJORANA and Gerda, the goal is a 1-ton experiment to explore the inverted hierarchy down to about $20 \mathrm{meV}$.

To achieve these sensitivities it is necessary to fabricate the detectors from germanium enriched to $86 \%$ in ${ }^{76} \mathrm{Ge}$. This material can be produced in Zelenogorsk, Russia, in the required quantities via centrifuge. The $\mathrm{Ge}$ metal is converted into a $\mathrm{GeF}_{4}$ gas that is very stable at high temperatures, but must be converted into an oxide or metal after centrifugation. This is the same type of starting material that was used in both the HeidelbergMoscow and IGEX experiments. No observable internal contamination of the Ge was found in either of those experiments.

The proposed MAJORANA experiment. The original Majorana concept was for eight modules with $571.1-\mathrm{kg}$ segmented Ge detectors each, for a total of $\sim 500 \mathrm{~kg}$ of Ge, enriched to $86 \%$ in ${ }^{76} \mathrm{Ge}$ (Gaitskell et al., 2003). The detectors within each module would be arranged in a hexagonal configuration of 19 towers, each three detectors high, inside of a copper cryostat. The cryostats will be electroformed as in the case of the IGEX cryostats. An intense $R \& D$ project is underway to reach the following levels of radio purity for $\mathrm{Cu}:{ }^{214} \mathrm{Bi}, 0.3 \mu \mathrm{Bq} / \mathrm{kg}$; and ${ }^{232} \mathrm{Th}, 0.1 \mu \mathrm{Bq} / \mathrm{kg}$. These two isotopes produce the most serious of backgrounds, for example, the 2615-keV $\gamma$ ray from ${ }^{208} \mathrm{Tl}$ at the end of the ${ }^{232} \mathrm{Th}$ chain. The background associated with this $\gamma$ ray is difficult to eliminate sufficiently by analysis as discussed below. For this reason, the reduction of thorium, as well as ${ }^{214} \mathrm{Bi}$, in the copper is a critical step.

The electroforming of the copper cryostat parts begins with the selection of pure materials. The copper is electroplated on stainless steel mandrels from a copper sulfate solution. The solution is prepared with semiconductor-grade acids. The copper sulfate is purified by recrystallization. This procedure was tested by artificially contaminating the copper sulfate, and then recrystallizing it several times, measuring the radioactivity at each step. The solution is constantly recirculated through microfilters to remove oxides and precipitates. It is also circulated through a barium scavenge filter to remove radium. The plating tanks have a cover gas to reduce oxides. The copper parts are machined periodi-
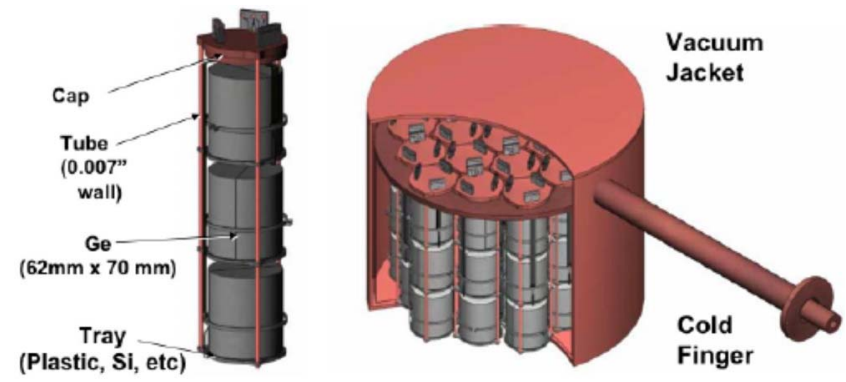

FIG. 11. (Color online) The left panel shows a concept drawing of 1 of 19 strings of Ge detectors that would be contained within a MAJORANA module shown in the right panel. Figure courtesy of the MAJORANA Collaboration.

cally to prevent the formation of porous copper, which can lead to vacuum leaks and poor structural strength. While this is a time consuming process, the payoff is copper cryostat parts with less than $1 \mu \mathrm{Bq} / \mathrm{kg}$ of ${ }^{232} \mathrm{Th}$.

Each module of 57 detectors comprises 19 threedetector towers as shown in Fig. 11. The final design of the individual Ge detectors has not yet been completed; however, the base plan has $1.1-\mathrm{kg}$ segmented detectors with a geometry similar to that of the Canberra CLOVER detectors. Some initial studies concerning the joint use of segmentation and pulse-shape analysis with these detectors has been encouraging (Elliott et al., 2006). Background events from ${ }^{56,57,60} \mathrm{Co},{ }^{65} \mathrm{Zn}$, and ${ }^{68} \mathrm{Ge}$, formed in the Ge crystals by spallation reactions from high-energy neutrons produced by cosmic-ray muons, can be identified event-by-event by the deposition of energy in more than one detector segment, and by pulseshape discrimination.

Another significant source of background is externally produced $\gamma$ rays. Gamma rays that can deposit $2-3 \mathrm{MeV}$ in a $\mathrm{Ge}$ detector most likely scatter several times before either being absorbed or leaving the crystal. This creates multiple sites where clouds of electrons and holes are formed. The result is a complex displacement current pulse that can be distinguished from one that results from a single-site event like that expected from $\beta \beta(0 \nu)$. Sample experimental pulses are shown in Fig. 12. The figure displays an experimental spectrum of background after segment cuts and pulse-shape discrimination have been applied. All $\gamma$-ray lines have been significantly reduced except for the $1593-\mathrm{keV}$ doubleescape-peak line of the $2615-\mathrm{keV} \gamma$ ray in ${ }^{208} \mathrm{Tl}$. This well-known line is from the single site creation of electron-positron pairs, in which both annihilation $\gamma$ rays completely escape the detector. One also sees that about $1 / 3$ of the continuum remains because it results from single Compton scatters.

Accordingly, by lowering the background in the copper cryostat parts, by carefully screening and selecting the front-end electronic parts for low background, and by applying segmentation and pulse-shape discrimination, the MAJORANA Collaboration hopes to reduce the background in the region of interest at $2039 \mathrm{keV}$ by a 

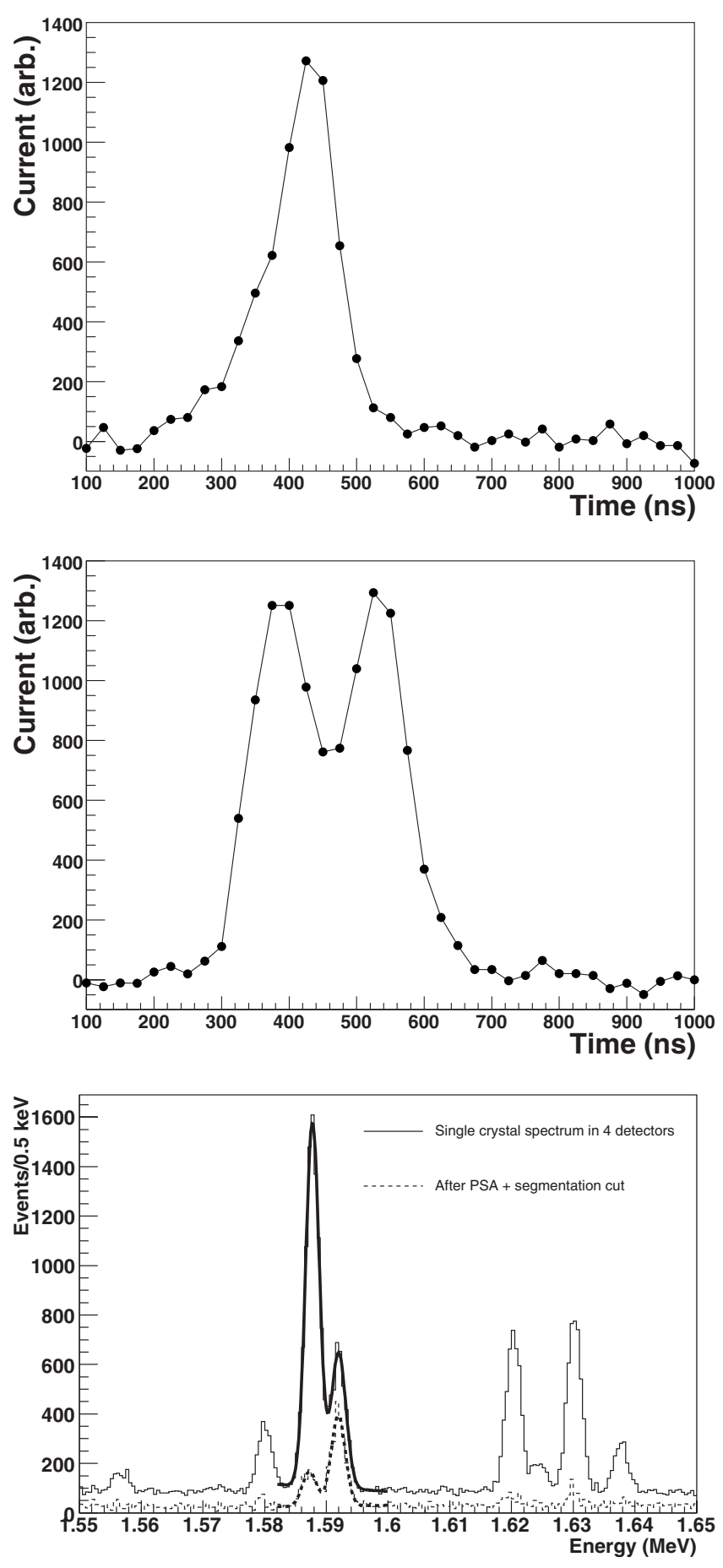

FIG. 12. The top panel shows the wave form of a candidate single-site energy deposit in a Ge detector. The middle panel shows a candidate multiple-site energy deposit of similar energy. The bottom panel shows how the spectrum in the region near $1.6 \mathrm{MeV}$ changes as pulse-shape analysis and segmentation cuts are employed. Figure courtesy of the MAJORANA Collaboration.

factor of 150 below that of IGEX or HeidelbergMoscow. The target half-life sensitivity, after an exposure of 0.46 tonne $\mathrm{yr}$, is $5.5 \times 10^{26} \mathrm{yr}$. If we use the central value of the nuclear structure factor, $F_{N}=1.22$ $\times 10^{-14} \mathrm{yr}$ (Rodin et al., 2003, 2006), the lifetime corre-

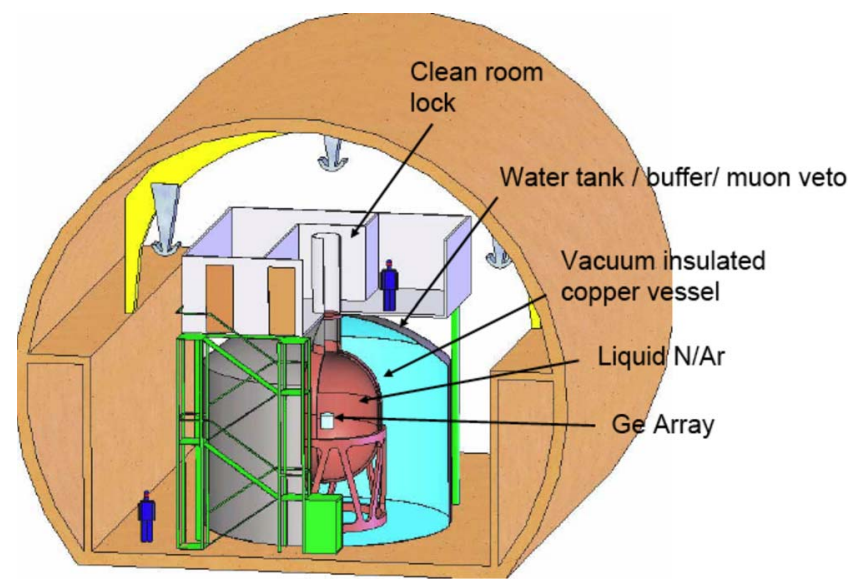

FIG. 13. (Color online) An artist's conception of the GERDA experiment. Figure courtesy of the GERDA Collaboration.

sponds to $\left\langle m_{\beta \beta}\right\rangle \sim 61 \mathrm{meV}$ to a $90 \% \mathrm{CL}$. If the claimed observation is correct (Klapdor-Kleingrothaus et al., 2004b), then this exposure would result in a measurement of the half-life with an accuracy of $10 \%$.

MAJORANA is a collaboration of about 70 physicists from 16 institutions in Canada, Japan, Russia, and the USA, and has been approved for major research and development.

The GERDA experiment (Germanium Detector Array). The Germanium Detector Array (GERDA) is a planned array of Ge detectors fabricated from germanium enriched to $86 \%$ in ${ }^{76} \mathrm{Ge}$, cooled and shielded by direct immersion in liquid argon or nitrogen (Abt et al., 2004; Schönert et al., 2005). The facility is presently under construction in the Laboratori Nazionali del Gran Sasso (LNGS) in Assergi, Italy. It is planned in three phases. The first phase will proceed with the five detectors from the Heidelberg-Moscow (HM) experiment, with $11.9 \mathrm{~kg}$ of enriched $\mathrm{Ge}$, and the three detectors from the IGEX experiment with $6 \mathrm{~kg}$ of enriched $\mathrm{Ge}$. All of these detectors are closed-end $p$-type semicoaxial detectors, and are unsegmented. In their copper cryostats, these detectors achieved background rates of $\approx 0.06$ count $/(\mathrm{keV} \mathrm{kg} \mathrm{yr})$ after pulse-shape discrimination that reduced the number of $\gamma$-ray events producing multisite pulses. The detectors have been underground since the early 1990s, the HM detectors in LNGS, and the IGEX detectors in the Laboratorio Subteranio de Canfranc in Spain. The goal of GERDA is to reduce the background to levels between 0.01 and 0.001 count / $(\mathrm{keV} \mathrm{kg} \mathrm{yr})$ in the initial phases via ultraclean materials, pulse-shape analysis, segmentation, and live and bulk shielding with liquid argon. An artist's conception of GERDA is shown in Fig. 13.

Phase I, comprising an active mass of $17.9 \mathrm{~kg}$, is supposed to achieve a half-life sensitivity of $\approx 3 \times 10^{25} \mathrm{yr}$ at $90 \%$ CL. For phase II, an additional $37.5 \mathrm{~kg}$ of Ge, enriched to $87 \%$ in ${ }^{76} \mathrm{Ge}$, has been delivered to the MaxPlanck-Institute in Munich in the form of $\mathrm{GeO}_{2}$. It will be stored underground until fabrication begins. The collaboration is testing one sixfold segmented $p$-type detec- 


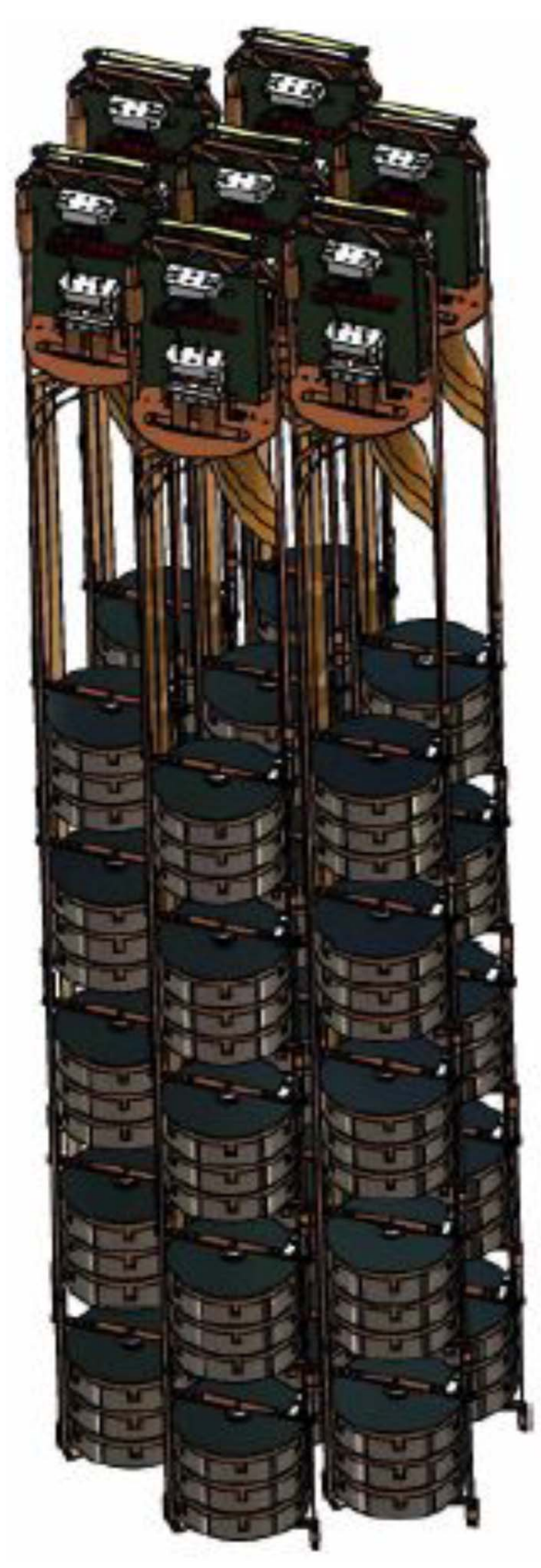

FIG. 14. (Color online) An artist's conception of the GERDA phase II tower. Figure courtesy of the GERDA Collaboration.

tor, and one 18-fold segmented $n$-type detector for possible use in phase II (Abt et al., 2007). The goal of phase II is to reach a half-life sensitivity of $\approx 1.4 \times 10^{26} \mathrm{yr}(90 \%$ $\mathrm{CL}$ ) and an exposure of $100 \mathrm{~kg}$ yr. If we use the central value of the nuclear structure factor $F_{N}=1.22 \times 10^{-14} \mathrm{yr}$ (Rodin et al., 2003, 2006), this corresponds to $\left\langle m_{\beta \beta}\right\rangle$ $\sim 124 \mathrm{meV}$. In the case that the claimed observation by Klapdor-Kleingrothaus et al. (2004b) is correct, phase I would observe it and phase II would make an accurate measurement of the lifetime. Figure 14 shows an artist's conception of a phase II detector tower that would hang directly in the liquid argon.

The GERDA cryostat is a vacuum-insulated stainless cryostat with an inner copper liner. The outer cylinder is
$4.2 \mathrm{~m}$ in diameter and $8.9 \mathrm{~m}$ long. The inner volume is $70 \mathrm{~m}^{3}$, the mass of the empty vessel is 25 metric tons, and it will contain 98 tons of liquid argon. There will be a clean room over the vessel and a rail system to lower and position the individual detector strings.

Monte Carlo calculations were performed in collaboration with members of the MAJORANA computation group to predict the background levels. GERDA will operate in Hall-A of the LNGS with an overburden of $\approx 3600$ mwe Accordingly it will have a muon veto shield to reduce backgrounds associated with cosmic-ray muons. Given the low- $Z$ material used as shielding in the vicinity of the germanium array, the muon-induced background will be $0.0001 \mathrm{count} /(\mathrm{keV} \mathrm{kg} \mathrm{yr})$ or less (Pandola et al., 2007). In addition to segmentation cuts, and pulse-shape analysis, anticoincidence signals between individual Ge detectors can be used to tag and eliminate external $\gamma$ rays. Coincidences from $\gamma$ rays in decay chains can be used to further reduce backgrounds. Finally, R\&D toward the use the scintillation light of liquid argon as an active veto system is underway. The technique may be implemented in a later phase of GERDA (Di Marco et al., 2007).

The ultimate goal of a world wide collaboration would be a half-life sensitivity of $10^{28} \mathrm{yr}$, corresponding to $\approx 25 \mathrm{meV}$. This would presumably be an experiment with the order of one ton of enriched germanium. This endeavor may very well be a collaboration between the GERDA and MAJORANA groups, depending on which technology proves to be the best with respect to background, detector stability, etc. There exists a memorandum of understanding to that effect, and it is clear that there will very probably be only one Ge experiment in the world of that magnitude.

GERDA is a collaboration of 80 physicists from 13 institutions in Belgium, Germany, Italy, Poland, and Russia. It was approved in November 2004, and is currently under construction.

\section{COBRA (Cadmium Telluride 0-neutrino Beta Decay Research Apparatus)}

COBRA (Zuber, 2001) is an R\&D program developing CdZnTe semiconductor crystal detectors (usually referred to as CZT detectors) for $\beta \beta(0 \nu)$. CZT detectors contain nine isotopes that undergo $\beta \beta$. The isotopes ${ }^{116} \mathrm{Cd},{ }^{130} \mathrm{Te},{ }^{114} \mathrm{Cd},{ }^{70} \mathrm{Zn}$, and ${ }^{128} \mathrm{Te}$ all undergo $\beta^{-} \beta^{-}$, whereas the isotopes ${ }^{64} \mathrm{Zn},{ }^{106} \mathrm{Cd},{ }^{108} \mathrm{Cd}$, and ${ }^{120} \mathrm{Te}$ undergo $\beta^{+} \beta^{+}, \beta^{+} \mathrm{EC}$, or EC-EC. The critical nucleus is ${ }^{116} \mathrm{Cd}$ as it has the highest $Q_{\beta \beta}$ and therefore the greatest sensitivity to $\beta \beta(0 \nu)$. Although the $Q_{\beta \beta}$ of ${ }^{130} \mathrm{Te}$ is also high, it is below that of ${ }^{116} \mathrm{Cd}$ and therefore the $\mathrm{Cd}$ $\beta \beta(2 \nu)$ would create a significant background for any $\beta \beta(0 \nu)$ study of Te. The isotopic abundance of ${ }^{116} \mathrm{Cd}$ is $7.5 \%$, however, necessitating enrichment for a competitive experiment. The existence of a number of $\beta^{+} \mathrm{EC}$ isotopes within the detector and the high granularity of the experiment permit a variety of studies in addition to the primary $\beta \beta(0 \nu)$. 


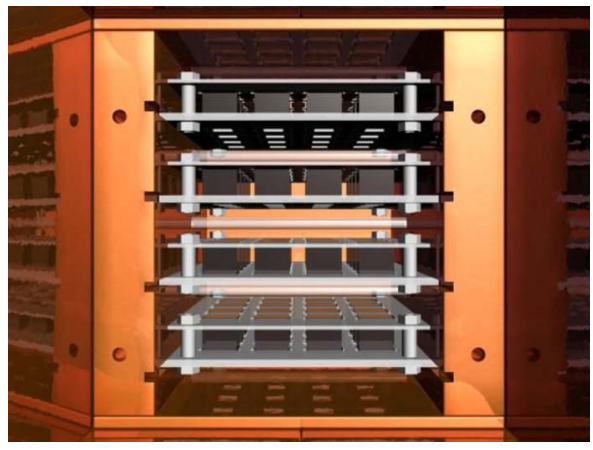

FIG. 15. (Color online) An artist's conception of COBRA 64detector setup. Figure courtesy of the COBRA Collaboration.

The proposal is to operate $640001-\mathrm{cm}^{3} \mathrm{CZT}$ detectors $\left({ }^{116} \mathrm{Cd}_{0.9} \mathrm{Zn}_{0.1} \mathrm{Te}\right)$ with a total mass of $418 \mathrm{~kg}$. The detectors would be fabricated with $\mathrm{Cd}$ enriched to $90 \%$ in isotope 116 , so about $44 \%$, or $183 \mathrm{~kg}$, of the detector mass is the isotope of interest. The energy resolution [full width at half maximum (FWHM)] of the co-planar grid $(\mathrm{CPG})$ detectors is better than $2 \%$ at the end point. If the background can be kept below 1 count/ ( $\mathrm{keV}$ tonne $\mathrm{yr}$ ), a half-life sensitivity of better than $10^{26} \mathrm{yr}$ is anticipated. To improve the resolution of the detectors, cooling and new grid designs are being investigated. The collaboration has operated a $2 \times 2$ detector prototype and is assembling a $4 \times 4 \times 4$ prototype array at Gran Sasso (Fig. 15), with the first 16 detectors running since summer 2006 (Fig. 16).

Initial data (Gößling et al., 2005) for the $2 \times 2$ detector array, including a measurement of the four-fold forbidden $\beta$ decay of ${ }^{113} \mathrm{Cd}$ and new limits on positron decay modes of $\beta \beta$, have been published. The collaboration is also studying pixellization of the detectors to improve spatial resolution within a single detector for background rejection by particle identification.

\section{MOON (Molybdenum Observatory of Neutrinos)}

The MOON experiment is unique because it has two applications. First, it is a tracking detector to search for the double beta decay of ${ }^{100} \mathrm{Mo}$ and other isotopes. The

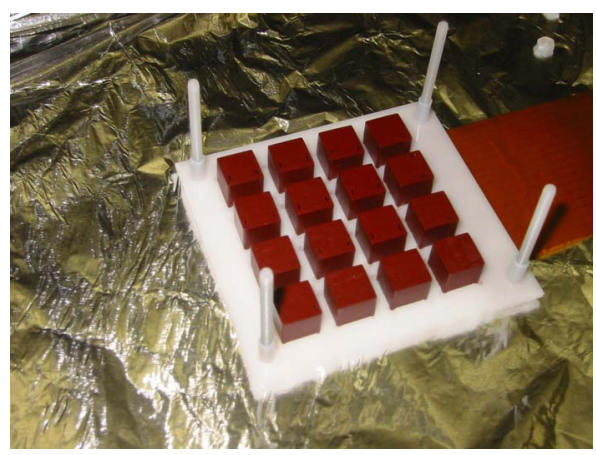

FIG. 16. (Color online) A photograph of a detector holder containing 16 detectors. Figure courtesy of the COBRA Collaboration. energy and angular correlations of individual $\beta$ rays are used to identify the $\nu$-mass term of the $\beta \beta(0 \nu)$ decay. In addition it will detect solar neutrinos, from the lowenergy $p p$ neutrinos all the way up to ${ }^{8} \mathrm{~B}$ neutrinos. This dual function results from the unique nuclear structure of the triplet ${ }^{100} \mathrm{Mo},{ }^{100} \mathrm{Tc}$, and ${ }^{100} \mathrm{Ru}$ (Ejiri et al., 2000; Ejiri, 2005). In this discussion we concentrate on MOON as a double beta decay experiment (Ejiri, 2006b; Nakamura et al., 2006, 2007).

The MOON Collaboration involves members from 12 institutions in Japan, the Czech Republic, Russia, and the US. The goal is to achieve an effective sensitivity to $\left\langle m_{\beta \beta}\right\rangle$ of $\sim 50 \mathrm{meV}$ with one ton of ${ }^{100} \mathrm{Mo}$. This isotope has a high $Q_{\beta \beta}(3034 \mathrm{keV})$, above the highest energy and troublesome $2615-\mathrm{keV} \gamma$ ray in ${ }^{208} \mathrm{Tl}$. It has a correspondingly large phase space factor, about seven times larger than that of ${ }^{76} \mathrm{Ge}$, and a natural isotopic abundance of $9.6 \%$. While its QRPA nuclear matrix element is slightly smaller than that of ${ }^{76} \mathrm{Ge}$, its predicted rate is 3.6 times faster (Rodin et al., 2006). On the other hand, for the value of $g_{p p}$ used in the calculations of Civitarese and Suhonen, this ratio is 5.84. Neither the old nor recent shell model calculations (Caurier et al., 1996; Poves et al., 2007) treat the case of ${ }^{100} \mathrm{Mo}$. The above two recent QRPA calculations differ by a factor of 1.6, and with other values of $g_{p p}$, as much as a factor of 4 . This makes a great difference in the predicted effectiveness of ${ }^{100} \mathrm{Mo} \beta \beta$ experiments. Thus depending on the chosen value for $g_{p p}$, the predicted $\beta \beta(0 \nu)$ rate might vary by a factor of 4. It should also be pointed out that the MOON technique, where the source does not comprise the detector, could also be used with other isotopes, ${ }^{82} \mathrm{Se}$ and ${ }^{150} \mathrm{Nd}$, for example. The choice is made by considering $M_{0 \nu}, Q_{\beta \beta}$, the $\beta \beta(2 \nu)$ rate, and other characteristics of a given isotope.

The experimental technique is based on the ELEGANT V design (Ejiri et al., 1991a, 1991b). The apparatus consists of multilayer modules, each with a thin film of enriched Mo, sandwiched between two position-sensitive detector planes (thin MWPC chambers or thin PL fibers) and two solid scintillator (plastic) plates, with all other modules acting as an active shield. Precise localization of the two $\beta$-ray tracks enables one to select true signals and reject background. In fact, background from radioactive impurities and from neutrons associated with cosmic-ray muons is evaluated by Monte Carlo simulations and found to be acceptable $[<0.3 /$ (tonne yr) $]$. One of the key challenges in this type of experiment is achieving adequate energy resolution to be able to recognize $\beta \beta(0 \nu)$ events from background, and also from the irreducible background from $\beta \beta(2 \nu)$ events. The half-life for $\beta \beta(2 \nu)$ of ${ }^{100} \mathrm{Mo}$ was measured very accurately by the NEMO experiment as $T_{1 / 2}^{2 v}$ $=7.11 \pm 0.02$ (stat) $\pm 0.54($ syst $) \times 10^{18} \mathrm{yr}$ (Arnold et al., 2005a). The theoretically predicted $T_{1 / 2}^{0 \nu}$ of ${ }^{100} \mathrm{Mo}$, for a $\left\langle m_{\beta \beta}\right\rangle=50 \mathrm{meV}$, is as long as $3.8 \times 10^{26} \mathrm{yr}$ (Rodin et al., 2006), although other $M_{0 \nu}$ calculations indicate $T_{1 / 2}^{0 \nu}$ nearer to $10^{26} \mathrm{yr}$. The $\beta \beta(2 \nu)$ rate is then between seven 


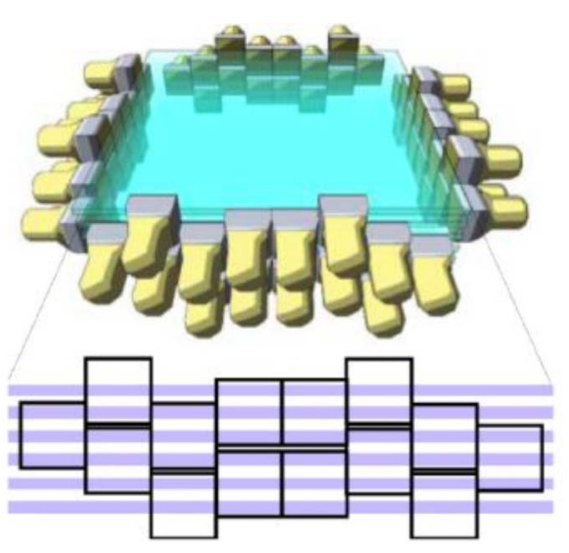

FIG. 17. (Color online) A schematic of the MOON-I scintillator layout. Figure courtesy of the MOON Collaboration.

and eight orders of magnitude faster than the predicted $\beta \beta(0 \nu)$ rate. Obviously, energy resolution is a key issue in all such experiments. In the case of MOON, it is being addressed by an intensive R\&D program (Nakamura et al., 2007).

The MOON-I prototype has the general features shown in Fig. 17. The three $48 \mathrm{~cm} \times 48 \mathrm{~cm}$ molybdenum films are about $47 \mathrm{~g}$ each and are enriched to $94.5 \%$ in ${ }^{100}$ Mo. They are supported and covered by aluminized Mylar films, $6 \mu \mathrm{g} / \mathrm{cm}^{2}$. This prevents optical cross talk between the scintillators. The six plastic scintillators are $53 \mathrm{~cm} \times 53 \mathrm{~cm} \times 1 \mathrm{~cm}$, and are viewed by 56 Hamamatsu, R6236-01 KMOD photomultiplier tubes that are relatively low in ${ }^{40} \mathrm{~K}$. They are optically coupled to the plastic scintillators with silicon cookies, which are made from silicon rubber $3 \mathrm{~mm}$ thick. The general geometry of the MOON-I configuration is shown in Fig. 18. The pattern of PMT coupling to the scintillators shown in Fig. 17 allows the hit scintillator to be identified by the PMT hit pattern. The measured energy resolution is $\sigma=4.8 \pm 0.2 \%(\Delta E / E)$ at $1 \mathrm{MeV}$. This would yield a $\sigma \approx 2.9 \%$ and FWHM of approximately $200 \mathrm{keV}$ at the $Q_{\beta \beta}$ of $3034 \mathrm{keV}$. The collaboration is attempting to improve it to $\sigma \approx 2.2 \%$ (as they have done in a small prototype) by adjusting the position dependent response.

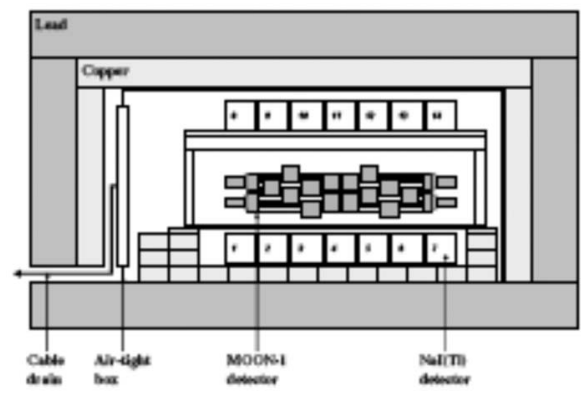

FIG. 18. A schematic of the MOON-I configuration. Figure courtesy of the MOON Collaboration.

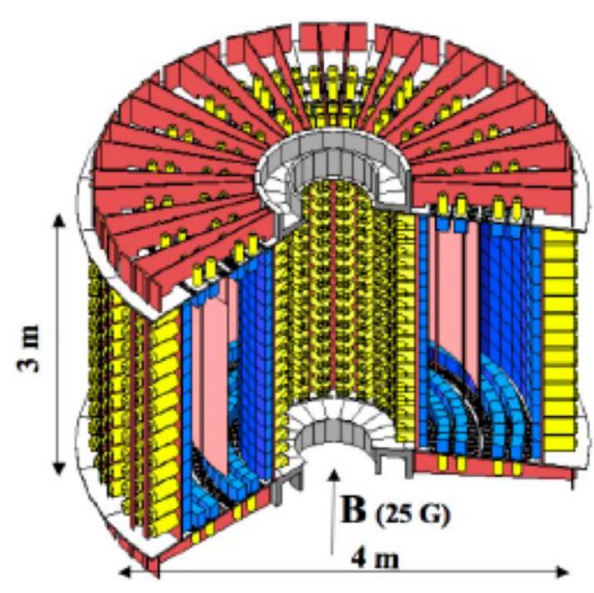

FIG. 19. (Color online) A schematic of the NEMO detector. Figure courtesy of the NEMO Collaboration.

The MOON-I experiment was performed with the detector inside the ELEGANT-V shield in the Oto underground Labotatory with an overburden of 1300 mwe (Ejiri et al., 1991a, 2001). At this level the muon flux was measured to be $4 \times 10^{-7} \mu /\left(\mathrm{cm}^{2} \mathrm{~s}\right)$. There are $\mathrm{NaI}(\mathrm{Tl})$ detectors above and below the MOON-I array to detect background $\gamma$ rays. The shield consists of $15 \mathrm{~cm}$ of lead outside of $10 \mathrm{~cm}$ of oxygen-free, high conductivity copper. The shield was flushed with dry $\mathrm{N}_{2}$ that reduced the activity from radon to a level of $125 \mathrm{mBq} / \mathrm{m}^{3}$.

The MOON research and development program continues with the main goals of reducing background as indicated by simulations (which have not yet been reported), improving the energy resolution, and improving the position resolution for tracking. The sensitivity of any ${ }^{100}$ Mo experiment to $\left\langle m_{\beta \beta}\right\rangle$ cannot really be determined until more reliable nuclear matrix elements are available. Experimental studies with charge exchange reactions are in progress for ${ }^{82} \mathrm{Se},{ }^{100} \mathrm{Mo}$, and ${ }^{150} \mathrm{Nd}$, which are candidates for use in MOON (Ejiri, 2000).

\section{NEMO-3 and SuperNEMO}

NEMO-3. The SuperNEMO detector (Barabash, 2004) will be a tracking detector, as is its currently operating predecessor, the NEMO-3 detector (Arnold et al., 2005b). We discuss the NEMO-3 experiment (Arnold et al., 2005a; Barabash, 2006b) and its results to provide background. NEMO-3 is the third generation of NEMO $\beta \beta$ detectors. An artist's conception is shown in Fig. 19. The detector has 20 segments of thin source planes, with a total area of $20 \mathrm{~m}^{2}$, that can support about $10 \mathrm{~kg}$ of source material. It has a three-dimensional readout wire drift chamber, with 6180 cells that operate in the Geiger mode for tracking. The detector gas is $\mathrm{He}$, with $4 \%$ ethyl alcohol, $1 \%$ argon, and $0.1 \% \mathrm{H}_{2} \mathrm{O}$. The tracking volumes are surrounded by 1940 plastic scientillator-block calorimeters. The scntillator detectors operate with thresholds of $\approx 30 \mathrm{keV}$, and have efficiencies of $50 \%$ for $1-\mathrm{MeV} \gamma$ rays. The energy resolutions range from $11 \%$ to $14.5 \% \mathrm{FWHM}$ at about $1 \mathrm{MeV}$. The resolution is one 
TABLE V. A comparison of the SuperNEMO design parameters with those of the NEMO detector.

\begin{tabular}{lcc}
\hline \hline NEMO & Detector/experiment & SuperNEMO \\
\hline${ }^{100} \mathrm{Mo}$ & isotope & ${ }^{82} \mathrm{Se}$ or ${ }^{150} \mathrm{Nd}$ \\
$7 \mathrm{~kg}$ & source mass & $100-200 \mathrm{~kg}$ \\
$8 \%$ & $\beta \beta(0 \nu)$ detection efficiency & $\approx 30 \%$ \\
${ }^{208} \mathrm{Tl}<20 \mu \mathrm{Bq} / \mathrm{kg}$ & external background & ${ }^{208} \mathrm{Tl}<2 \mu \mathrm{Bq} / \mathrm{kg}$ \\
${ }^{214} \mathrm{Bi}<300 \mu \mathrm{Bq} / \mathrm{kg}$ & in the source foil & for ${ }^{82} \mathrm{Se}:{ }^{214} \mathrm{Bi}<10 \mu \mathrm{Bq} / \mathrm{kg}$ \\
$8 \%$ at $\approx 3 \mathrm{MeV}$ & energy resolution, FWHM & $4 \%$ at $3 \mathrm{MeV}$ \\
$2 \times 10^{24} \mathrm{yr}$ & $T_{1 / 2}^{0 \nu}$ sensitivity & $>10^{26} \mathrm{yr}$ \\
$0.3-1.3 \mathrm{eV}$ & $\left\langle m_{\beta \beta}\right\rangle$ sensitivity & $<0.05-0.1 \mathrm{eV}$ \\
\hline \hline
\end{tabular}

parameter that will be given a lot of attention in the design and construction of SuperNEMO. Good energy resolution is critical for the discovery of processes with long half-lives. The source planes hang vertically in a cylindrical geometry inside of a magnetic solenoid that generates $a \sim 25 \mathrm{G}$ magnetic induction field. Tracking in the magnetic field allows the differentiation between electron and positron tracks, with only a $3 \%$ chance of confusing the two.

The detector is surrounded by $18 \mathrm{~cm}$ of lowbackground iron to reduce the external $\gamma$-ray flux. Fast neutrons from the laboratory environment are suppressed by an external shield of water, and by wood and polyethylene plates. NEMO-3 is located in the Modane Underground Laboratory in Frejus, France with an overburden of 4800 mwe. The air in the experimental area is constantly flushed and has a radon-free purification system serving the detector volume. It reduces the radon to $\approx 200 \mathrm{mBq} / \mathrm{m}^{3}$, and has a capacity of $150 \mathrm{~m}^{3} / \mathrm{h}$. The measured radon activity in the detector was $4-5 \mathrm{mBq} / \mathrm{m}^{3}$.

One of the great advantages of the NEMO-3 detector is that it can make measurements on many different nuclei, and in fact has made measurements on seven isotopes, and two blanks, simultaneously. The isotopes were ${ }^{100} \mathrm{Mo}(6.914 \mathrm{~kg}),{ }^{85} \mathrm{Se}(932 \mathrm{~g}),{ }^{\text {nat }} \mathrm{Cu}(621 \mathrm{~g}),{ }^{\text {nat }} \mathrm{Te}$ (491 g), ${ }^{130} \mathrm{Te}(454 \mathrm{~g}),{ }^{116} \mathrm{Cd}(405 \mathrm{~g}),{ }^{150} \mathrm{Nd}(37 \mathrm{~g}),{ }^{96} \mathrm{Zr}$ $(9.4 \mathrm{~g})$, and ${ }^{48} \mathrm{Ca}(7 \mathrm{~g})$. The ${ }^{\mathrm{nat}} \mathrm{Te}$ and ${ }^{\text {nat }} \mathrm{Cu}$ were used to measure the external background.

The measurement of the decay of ${ }^{100} \mathrm{Mo}$ made with NEMO-3 truly sets a standard for such measurements (Arnold et al., 2005a). The result was $T_{1 / 2}^{2 v}$ $=7.11 \pm 0.02$ (stat) \pm 0.54 (syst) $\times 10^{18} \mathrm{yr}$. New bounds were set on $\beta \beta(0 \nu)$ (Barabash, 2006b): $T_{1 / 2}^{0 \nu}>5.8 \times 10^{23} \mathrm{yr}$ for ${ }^{100} \mathrm{Mo}$, and $2.1 \times 10^{23} \mathrm{yr}$ for ${ }^{85} \mathrm{Se}(90 \%$ confidence level). The bounds on the effective Majorana mass of the electron neutrino are $\left\langle m_{\beta \beta}\right\rangle<0.6-2.7 \mathrm{eV}$ for ${ }^{100} \mathrm{Mo}$ and $1.2-3.2 \mathrm{eV}$ for ${ }^{85} \mathrm{Se}$. The NEMO-3 detector has been operating since February 2003. The collaboration projects that the sensitivity to $T_{1 / 2}^{0 \nu}$ of ${ }^{100} \mathrm{Mo}$ will be $2 \times 10^{24} \mathrm{yr}$ by the end of 2009. This would reduce the bound on $\left\langle m_{\beta \beta}\right\rangle$ to $<0.34-1.34 \mathrm{eV}$. It is clear that by that time the detector will have been operating for seven years the resulting sensitivity will not be competitive with next-generation experiments that are designed to probe the invertedhierarchy mass scale. To remedy that situation, the SuperNEMO Collaboration was formed.

SuperNEMO. This proposed experiment is a vastly expanded tracking chamber of a modular design. The parent isotope will be either ${ }^{85} \mathrm{Se}$ or ${ }^{150} \mathrm{Nd}$. At this time there does not exist a source of kilogram quantities of $\mathrm{Nd}$ isotopically enriched in ${ }^{150} \mathrm{Nd}$, whereas ${ }^{85} \mathrm{Se}$ has been enriched by the gas centrifuge technique in Russia. In Table V we compare the experimental parameters of NEMO-3 to those of SuperNEMO.

Currently SuperNEMO is a collaboration of 26 institutes in 11 countries. An artist's conception of the detector modules is shown in Fig. 20. The detector will comprise 20 modules. Each module is designed to hold $5 \mathrm{~kg}$ of enriched isotope; each has $12 \mathrm{~m}^{2}$ of tracking volume, with 3000 channels of readout and 1000 photomultiplier

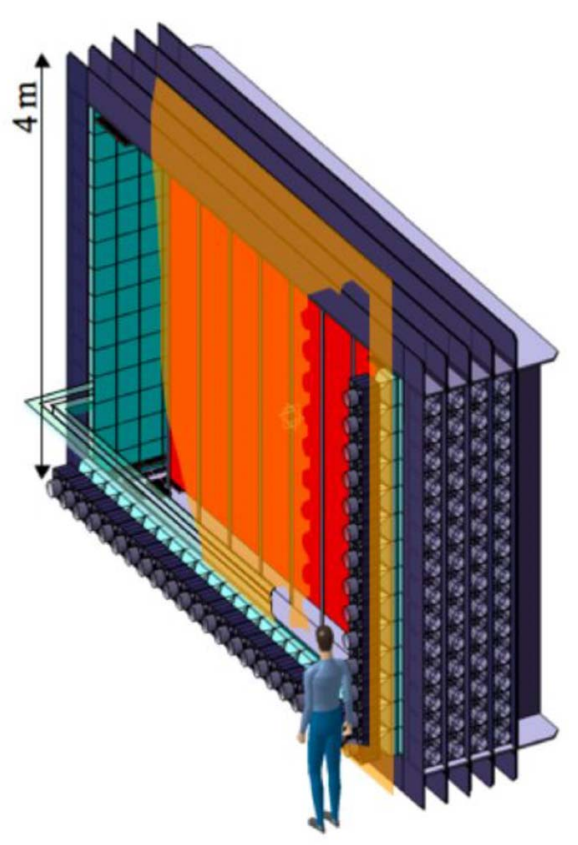

FIG. 20. (Color online) A schematic of a SuperNEMO module. Figure courtesy of the NEMO Collaboration. 
tubes. The total detector will have 60000 channels of drift chamber readout, and 20000 photomultiplier tubes.

One of the key issues is the background from the decay of ${ }^{214} \mathrm{Bi}$ to ${ }^{214} \mathrm{Po}$ in the ${ }^{238} \mathrm{U}$ chain and ${ }^{212} \mathrm{Bi}$ to ${ }^{212} \mathrm{Po}$ in the ${ }^{232} \mathrm{Th}$ chain. These are referred to as the BiPo backgrounds and a prototype-test detector named BiPo is installed in the Laboratorio Subteranio de Canfranc (LSC) in Canfranc, Spain to test the source foils. The BiPo detector comprises two modules. The intent is to test each source foil for the BiPo backgrounds before installation in SuperNEMO. The plan is to measure a $5-\mathrm{kg}$ foil every month with a sensitivity of ${ }^{208} \mathrm{Tl}$ $<2 \mu \mathrm{Bq} / \mathrm{kg}$ and ${ }^{214} \mathrm{Bi}<10 \mu \mathrm{Bq} / \mathrm{kg}$.

The proposed schedule is as follows: BiPo1 will run until 2008; BiPo will be under construction from mid 2007 until mid 2009. The final BiPo will be installed in LSC at the beginning of 2009. The construction of the first SuperNEMO module will start in early 2009 and be completed in mid 2010. The BiPo tests will continue throughout the construction of SuperNEMO. The full detector is scheduled to start operating at the beginning of 2012.

\section{CANDLES (calcium fluoride for studies of neutrinos and dark matters by low energy spectrometer)}

The proposed CANDLES experiment (Umehara et al., 2006) is based on undoped $\mathrm{CaF}_{2}$ scintillators surrounded by a $4 \pi$ liquid scintillator live shield. The scintillation light from pure $\mathrm{CaF}_{2}$ crystals is in the ultraviolet region of the spectrum and has a quite long decay time $(1 \mu \mathrm{s})$ although the liquid scintillator has a very short decay time ( $\sim 10 \mathrm{~ns})$. One can employ this difference to reject background signals from the liquid scintillator. A wavelength shifter will be added to the liquid scintillator to match the response of the photomultiplier tubes. The $\mathrm{CaF}_{2}$ crystals are made with natural-abundance calcium which is $0.187 \%{ }^{48} \mathrm{Ca}$. The end-point energy of ${ }^{48} \mathrm{Ca}$, 4.27 MeV, is the largest of all the $\beta \beta$ candidates, well above the highest $\gamma$ ray from natural radioactivity, namely, the $2615-\mathrm{keV} \gamma$ ray in the decay of ${ }^{208} \mathrm{Tl}$. It is interesting to note that ${ }^{48} \mathrm{Ca}$ is a good candidate for shell model analysis of the $\beta \beta$ matrix elements.

The CANDLES project follows the ELEGANT VI experiments performed with $\mathrm{CaF}_{2}$ scintillation detectors made with europium-doped $\mathrm{CaF}_{2}(\mathrm{Eu})$. In the ELEGANT VI detector there were $23 \mathrm{CaF}_{2}(\mathrm{Eu})$ crystals, each $4.5 \times 4.5 \times 4.5 \mathrm{~cm}^{3}(290 \mathrm{~g})$, surrounded by 46 $\mathrm{CaF}_{2}$ (pure) crystals that acted as a shield and as light guides. There was $6.7 \mathrm{~kg}$ of $\mathrm{CaF}_{2}$ containing $6.4 \mathrm{~g}$ of ${ }^{48} \mathrm{Ca}$. This in turn was surrounded by $38 \mathrm{CsI}(\mathrm{Tl})$ crystals, each $6.5 \times 6.5 \times 25 \mathrm{~cm}^{3}$, to act as a live shield. The entire array was placed inside a bulk shield of copper, an airtight box, lead, Li-loaded paraffin and Cd, and B-loaded water. The experiment was located in the Oto Cosmo Observatory with an overburden of 1400 mwe. The energy resolution was $3.1 \%$ at $4271 \mathrm{keV}$, and the detection efficiency was $\approx 49 \%$. The experiment resulted in a new bound for $T_{1 / 2}^{0 \nu}\left({ }^{48} \mathrm{Ca}\right)>1.8 \times 10^{22} \mathrm{yr}($ Ogawa et al., 2004).

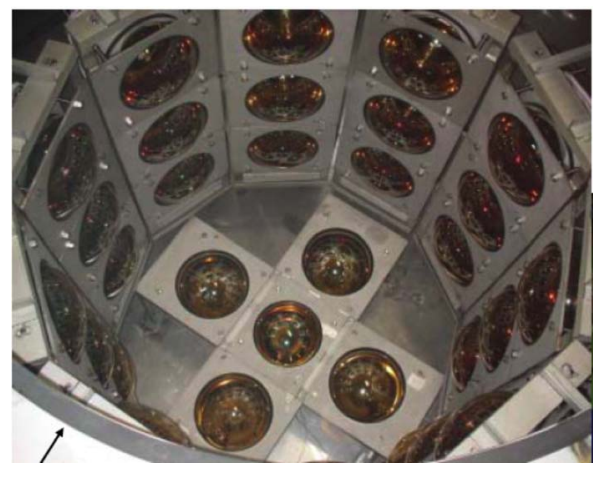

FIG. 21. (Color online) A photograph of the CANDLES-III installation. Figure courtesy of the CANDLES Collaboration.

According to recent shell model calculations (Poves $e t$ al., 2007), $\left\langle m_{\beta \beta}\right\rangle<23 \mathrm{eV}$. It will require several ton years for such an experiment to be competitive. Currently CANDLES-III is under construction with 60 crystals for a total mass of $191 \mathrm{~kg}$ at a sea level lab in Osaka (see Fig. 21).

The proposed CANDLES detector will comprise several tons of $\mathrm{CaF}_{2}$ (pure) detectors, with almost no background, immersed in liquid scintillator that will act as a veto detector. The target parameters for a 6.4-ton CANDLES are $<3 \mu \mathrm{Bq} / \mathrm{kg}$ of both ${ }^{212,214} \mathrm{Bi}, 3.5 \%$ FWHM resolution, and $6 \mathrm{yr}$ of counting with a total background of 0.3 per yr under the expected $\beta \beta(0 \nu)$ peak. The target sensitivity for this phase is $\left\langle m_{\beta \beta}\right\rangle$ $\approx 0.1 \mathrm{eV}$. According to shell model calculations (Poves et al., 2007), this would require a sensitivity of $T_{1 / 2}^{0 \nu}$ $\sim 7.2 \times 10^{26} \mathrm{yr}$.

The shell model calculations predict a value for the nuclear structure factor $F_{N}\left({ }^{48} \mathrm{Ca}\right)=3.61 \times 10^{-14} / \mathrm{yr}$. The same model predicts $F_{N}\left({ }^{76} \mathrm{Ge}\right)=3.22 \times 10^{-14} / \mathrm{yr}$ and $F_{N}\left({ }^{130} \mathrm{Te}\right)=2.13 \times 10^{-13} / \mathrm{yr}$. One can immediately appreciate the challenge of performing an experiment with natural-abundance calcium. One would need about 400 times as many $\mathrm{Ca}$ atoms as $\mathrm{Ge}$ atoms, enriched to $86 \%$ in ${ }^{76} \mathrm{Ge}$, to have the same decay rate. In comparison with ${ }^{130} \mathrm{Te}$, one would need about 1000 times the natural abundance $\mathrm{Ca}$ atoms as natural abundance of Te atoms to have the same theoretical decay rates. The collaboration is investigating techniques for enriching $\mathrm{Ca}$.

\section{Other proposals}

CARVEL (Zdesenko et al., 2005) (calcium research for very low neutrino mass) is a proposal to use isotopically enriched ${ }^{48} \mathrm{CaWO}_{4}$ crystal scintillators to search for $\beta \beta(0 \nu)$ of ${ }^{48} \mathrm{Ca}$. These scintillators, with natural calcium, have been tested for energy resolution, internal background, and pulse-shape discrimination that enables the discrimination between alpha and gamma ray events. The energy resolution at $2615 \mathrm{keV}$ is $3.8 \%(\approx 100 \mathrm{keV})$ which compares well with tracking detectors. The total background between 3.8 and $5.4 \mathrm{MeV}$, after the elimination of $\alpha$ background by pulse-shape discrimination, was 
0.07 count/( keV kg yr). With these parameters it was determined that with $\approx 100 \mathrm{~kg}$ of ${ }^{48} \mathrm{CaWO}_{4}$, enriched to $80 \%$ in ${ }^{48} \mathrm{Ca}$, the half-life sensitivity would be $T_{1 / 2}^{0 \nu}\left({ }^{48} \mathrm{Ca}\right) \sim 10^{27} \mathrm{yr}$. This corresponds to a sensitivity in effective neutrino mass of $\left\langle m_{\beta \beta}\right\rangle \sim 55 \mathrm{meV}$ (Rodin et al., 2006). With one ton of this material, it was estimated that a half-life sensitivity of $10^{28} \mathrm{yr}$ could be reached, corresponding to $\left\langle m_{\beta \beta}\right\rangle \sim 0.02 \mathrm{eV}$. The difficulty with this proposal is that there is no source of $\mathrm{kg}$ quantities of $\mathrm{Ca}$ enriched in ${ }^{48} \mathrm{Ca}$. While in principle, $\mathrm{Ca}$ can be enriched by atomic vapor laser isotope Separation (AVLIS), the effort and cost scale as the ratio of the final to initial abundance. This cost would be more than 35 times that for enriching an element with a $7 \%$ natural abundance (e.g., ${ }^{76} \mathrm{Ge}$ ). This is a challenge for a ton-scale experiment.

DCBA (Ishihara et al., 2000) (drift chamber beta ray analyzer) is designed to search for the $\beta \beta(0 \nu)$ of ${ }^{150} \mathrm{Nd}$. It comprises tracking chambers, a solenoid magnet, and a cosmic-ray veto detector. The source foils are vertical with a series of high-voltage anode wires parallel to the source and the magnetic field. As $\beta$ particles are emitted they are given an extra velocity perpendicular to the magnetic field, and are curved as they pass through the 1-atm He gas mixture. The anode wires detect the drift electrons, while the cathode wires across the chamber gather the drifted ions. The detector can measure the momentum of each $\beta$ particle and can determine the vertex. Drift electrons generate avalanche electrons on the anode wire plane. The pattern of avalanche ions and the time of each signal are recorded with flash analogto-digital converters (ADCs). From these data, the $x$ position of the origin of the track is determined. The $y$ position is fixed by the responding anode wire. The position on the anode wire of the avalanche determines the $z$ position with pickup wires, which are located transversely near anode wires and pick up signals induced by the avalanche. Each electron track is reconstructed in three dimensions, and the momentum and kinetic energy are obtained from the curvature and pitch angle. DCBA-I will contain natural $\mathrm{Nd}$ and will have $\approx 3.8$ $\times 10^{22}$ atoms of ${ }^{150} \mathrm{Nd}(5.6 \%$ natural abundance $)$. It is expected to have a background of $<0.1$ count/( $\mathrm{keV}$ yr). It will have an energy resolution of $\approx 100 \mathrm{keV}$. DCBA-II is planned to have $4.5 \times 10^{25}$ atoms of ${ }^{150} \mathrm{Nd}$, in the form of $\mathrm{Nd}_{2} \mathrm{O}_{3}$ with $\mathrm{Nd}$ enriched to $90 \%$ in ${ }^{150} \mathrm{Nd}$. The expected half-life sensitivity is $4 \times 10^{24} \mathrm{yr}$. According to recent QRPA calculations (Rodin et al., 2006) this would correspond to $\left\langle m_{\beta \beta}\right\rangle \sim 0.12 \mathrm{eV}$. The R\&D for this detector is being carried out at KEK with a test prototype DCBA-T. On the basis of that R\&D a new project has been proposed, temporally named MTD (magnetic tracking detector) (Ishihara, 2007). The detection principle is the same as in DCBA, but the amount of source installed is much larger. One module of MTD will contain natural $\mathrm{Nd}$ and will have $\approx 6.7 \times 10^{24}$ atoms of ${ }^{150} \mathrm{Nd}$ (5.6\% natural abundance) in the first experimental phase. The natural source plates will be replaced with enriched material in the second phase. The enrichment of ${ }^{150} \mathrm{Nd}$ is planned on a large scale with an international collaboration.

The CAMEO proposal (Bellini et al., 2001) would install $1000 \mathrm{~kg}$ of ${ }^{116} \mathrm{CdWO}_{4}$ crystals in the liquid scintillator of BOREXINO (Bellini et al., 1996) after its solar neutrino program is complete. The described program would begin $(\mathrm{CAMEO}-\mathrm{I})$ with thin $\left(\approx 15 \mathrm{mg} / \mathrm{cm}^{2}\right)$ metal isotopically enriched Mo sheets sandwiched between plastic scintillator and installed within the scintillator of the BOREXINO Counting Test Facility (CTF). Better sensitivity could be achieved, however, with an active source configuration of $\mathrm{CdWO}_{4}$ crystals. For CAMEOII, $100 \mathrm{~kg}$ of crystals would be installed in the CTF. Measurements of the intrinsic background of the crystals lead to an estimated $T_{1 / 2}^{0 \nu}$ sensitivity of $\approx 10^{26} \mathrm{yr}$. Installing 1 ton of crystals into the BOREXINO detector could allow a sensitivity of $10^{27} \mathrm{yr}$. Because of the focus on solar neutrinos at BOREXINO, this program is not currently active.

Another proposal that would exploit the BOREXINO detector or the CTF is to dissolve ${ }^{136} \mathrm{Xe}$ in the scintillator (Caccianiga and Giammarchi, 2001). In the most ambitious design, a vessel of radius $2.7 \mathrm{~m}$ containing $\mathrm{Xe}$ and scintillator is itself contained when the BOREXINO vessel (radius $4.25 \mathrm{~m}$ ) filled only with scintillator. Outside this scintillating region is a pseudocumene buffer. About $2 \%$ by weight $\mathrm{Xe}$ is soluble in scintillator with an appreciable temperature dependence, so an estimated $1565 \mathrm{~kg}$ of Xe could be used at $15^{\circ} \mathrm{C}$. Simulations indicate that phototube activity and $\beta \beta(2 \nu)$ will dominate the background, resulting in a $T_{1 / 2}^{0 \nu}$ sensitivity of $2 \times 10^{26} \mathrm{yr}$. This program is currently inactive.

The XMASS experiment (Takeuchi, 2008) [xenon detector for weakly interacting massive particles (WIMP)] is a dark-matter search using liquid $\mathrm{Xe}$ as a target for WIMP detection via nuclear scattering. The present detector uses $100 \mathrm{~kg}$ of natural Xe with a fiducial volume of $3 \mathrm{~kg}$ viewed by phototubes. It is planned to expand this to 1 ton and even possibly to 20 tons. This final configuration would have a fiducial volume of 10 tons and would be used to study dark matter and solar neutrinos. With such a large mass of $\mathrm{Xe}$, the sensitivity to $\beta \beta(0 \nu)$ is clearly of interest. The self-shielding of Xe is highly effective at reducing the backgrounds at low energies $(<500 \mathrm{keV})$ of interest to solar neutrinos and dark matter. However, it is less successful at the higher energy appropriate for $\beta \beta(0 \nu)$. Present efforts are aimed at reducing the backgrounds for the dark-matter search. Results from this $100-\mathrm{kg}$ prototype have shown that the dark matter configuration will not work for $\beta \beta$, which will require a dedicated design. Work toward such a design is not a current priority of the collaboration.

There are two proposals to use Ce-doped GSO scintillating crystals $\left(\mathrm{Gd}_{2} \mathrm{SiO}_{5}: \mathrm{Ce}\right)$ to search for $\beta \beta(0 \nu)$ in ${ }^{160} \mathrm{Gd}\left(Q_{\beta \beta}=1.73 \mathrm{MeV}\right)$. In one research effort, a $1.744-\mathrm{kg}$ GSO crystal was used (Wang et al., 2002) to study backgrounds. This crystal, coupled to a 2-in. phototube, was wrapped in Teflon and black vinyl tape. The 
whole crystal was inserted into a well-type $\mathrm{NaI}$ crystal. The system was contained within a $\mathrm{Pb}$ shield and a plastic-scintillator cosmic-ray veto. The whole setup was situated in the bottom floor of a seven-story building (12 mwe overburden). The crystal was found to have a substantial contamination from $\mathrm{U}$ - and Th-chain isotopes, which were not in equilibrium. In addition, $\alpha$ decays of ${ }^{152} \mathrm{Gd}$ were present. This program is no longer being pursued. GSO crystals were also used in an experiment at the Solotvina Underground Laboratory (Danevich, 2001) at a depth of $\approx 1000$ mwe. In this test, a $635-\mathrm{g}$ crystal was joined to a phototube by a $18.2-\mathrm{cm}$ plastic light guide. A passive shield of $\mathrm{Cu}, \mathrm{Hg}$, and $\mathrm{Pb}$ surrounded the detector. Internal radioactive contaminants within the crystal were the limitation of this experiment, although the detector was cleaner than that discussed above. This collaboration proposed putting GSO crystals within a large volume of liquid scintillator (an idea similar to that of the CAMEO project) to help control the background. Background levels will be a challenge for this technology. Although GSO crystals are costly, the high isotopic abundance of ${ }^{160} \mathrm{Gd}(22 \%)$ would permit the use of natural $\mathrm{Gd}$, which would be a great relative cost reduction compared to other proposals, assuming that pulse shape techniques could be used to suppress backgrounds. A modest R\&D program continues on this project.

Finally, there is also a proposal (Chen, 2005) to use the Sudbury Neutrino Observatory (SNO) (Boger et al., 2000) for $\beta \beta$, now that it has completed its solar neutrino studies. The plan for the SNO detector is to replace the heavy water with scintillator, beginning a program of low-energy solar-neutrino studies and longbaseline-oscillation studies of reactor neutrinos, geoneutrinos, and $\beta \beta$. The parent isotope, situated in the liquid scintillator either as nanoparticles, dissolved chemicals, or as absorbed gas, can be used as a source, with a Nd-loaded liquid scintillator as the leading candidate. The scintillator configuration is referred to as $\mathrm{SNO}+$. A $1 \%$ loading of natural $\mathrm{Nd}$ in the scintillator would provide 10 tons of $\mathrm{Nd}$, corresponding to more than $500 \mathrm{~kg}$ of ${ }^{150} \mathrm{Nd}$ isotope. Simulations of this configuration indicate that $\beta \beta(2 \nu)$ will be the dominant background because of the limited energy resolution. However, if $\left\langle m_{\beta \beta}\right\rangle$ is in the degenerate mass-scale region, the extremely high count rate from this large isotopic mass would permit a statistical separation of $\beta \beta(2 \nu)$ and $\beta \beta(0 \nu)$. A development program for $\mathrm{SNO}+$ is proceeding.

There are a number of research and development projects involving other crystals that could be candidates for $\beta \beta$ detectors. The crystals under investigation are $\mathrm{CdWO}_{4}$ (Bardelli et al., 2006), $\mathrm{PbWO}_{4}$ (Danevich et al., 2006), YAG:Nd (Danevich et al., 2005a), and $\mathrm{ZnWO}_{4}$ (Danevich et al., 2005b). A detector based on high pressure Xe gas is also being considered (Nygren, 2007).

\section{CONCLUSIONS}

The future for $\beta \beta$ is exciting. Technologies in hand will allow us to measure at intriguing neutrino-mass levels. Technological progress is rapid, and developments in theory are improving our ability to interpret the measurements. Strong $\beta \beta$ programs in both particle and nuclear physics are the result.

The answer to the question "Is the neutrino its own antiparticle?" is critical for theories of particle mass. It is also needed to help uncover the reasons matter dominates over antimatter in our Universe. The neutrino mass will not only tell us about the high scale at which the standard model breaks down (e.g., through the seesaw), but also will have implications for the large-scale structure of the Universe. Finally, lepton-number violaton is significant in its own right.

$\beta \beta(0 \nu)$ is the only practical method for investigating the particle-antiparticle question. And if the neutrino is its own antiparticle, $\beta \beta(0 \nu)$ will have the best sensitivity to neutrino mass of any laboratory technique. The $\beta \beta$ program outlined here, consisting of several experimental results, will therefore greatly influence the widerparticle physics endeavor.

Questions about how best to calculate the nuclear matrix elements have led to developments in nuclear theory, despite the preoccupation of most theorists with other problems. Large-scale shell model codes are increasing in power, in part because of the need for better matrix elements. And a variety of experiments on nuclei are planned to constrain those same matrix elements. We should see some reduction in their uncertainties.

Experimental progress in $\beta \beta(0 \nu)$ has also led to improved material-purification techniques such as $\mathrm{Cu}$ electroforming, and to ongoing improvement in assay techniques such as direct $\gamma$-ray counting and inductively coupled plasma-mass spectroscopy. The need for effective detectors has led to many improvements in semiconductor (e.g., Ge, CZT) and bolometer technologies that are now finding application in basic science, medicine, and homeland security. As researchers strive for 1-ton $\beta \beta$ experiments, advances in areas such as isotope enrichment and detector production should follow.

Overall, the program in $\beta \beta$ has been and will continue to be very fruitful.

Note added in proof. Recently Poves et al. (2007) presented slightly different values for the nuclear matrix elements than those that appeared in the original paper.

\section{ACKNOWLEDGMENTS}

The authors gratefully acknowledge useful comments from Alexander Barabash, Mark Chen, Hiro Ejiri, Gerry Garvey, Ryuta Hazama, Nobuhiro Ishihara, Andreas Piepke, Yoichiro Suzuki, Henry Wong, and Kai Zuber. A careful reading of the manuscript by Michael Smy, Hank Sobel, Vladimir Tretyak, and Petr Vogel is greatly appreciated. The work was supported by U.S. Department of Energy Grant No. DE-FG0297ER41019, National Science Foundation Grants No. 
PHY-0139294 and No. PHY-0500337, and the Los Alamos National Laboratory Directed Research and Development Program.

\section{REFERENCES}

Aalseth, C. E., et al., (IGEX), 2002a, Phys. Rev. D 65, 092007. Aalseth, C. E., et al., 2002b, Mod. Phys. Lett. A 17, 1475. Aalseth, C. E., et al., (IGEX), 2004, Phys. Rev. D 70, 078302. Abada, A., and G. Bhattacharyya, 2003, Phys. Rev. D 68, 033004.

Abt, I., et al., (GERDA), 2004, e-print arXiv:hep-ex/0404039.

Abt, I., et al., 2007, Nucl. Instrum. Methods Phys. Res. A 570, 479 .

Aguilar, A., et al., 2001, Phys. Rev. D 64, 112007.

Aguilar-Arevalo, A. A., et al., 2007, e-print arXiv:0704.1500.

Aharmin, B., et al., 2005, Phys. Rev. C 72, 055502.

Ali, A., A. V. Borisov, and D. V. Zhuridov, 2006, e-print arXiv:hep-ph/0606072.

Allessandrello, A., et al., 1997, IEEE Trans. Nucl. Sci. 44, 416.

Allessandrello, A., et al., 1998, Nucl. Instrum. Methods Phys.

Res. A 412, 454.

Allessandrello, A., et al., 1999, J. Phys. D 32, 3099.

Alvarez-Rodriguez, R., P. Sarriguren, E. M. de Guerra, L. Pacearescu, A. Faessler, and F. Šimkovic, 2006, Prog. Part. Nucl. Phys. 57, 251.

Amos, K., A. Faessler, and V. Rodin, 2007, e-print arXiv:nuclth/0702016.

Apollonio, M., et al., 1999, Phys. Lett. B 466, 415; 472, 434(E) (1999).

Ardito, R., et al., 2005, e-print arXiv:hep-ex/0501010.

Arnaboldi, C., et al., 2003, Phys. Lett. B 557, 167.

Arnaboldi, C., et al., 2004, Nucl. Instrum. Methods Phys. Res. A 518, 775.

Arnaboldi, C., et al., 2005, Phys. Rev. Lett. 95, 142501.

Arnaboldi, C., et al., 2007, private communication.

Arnold, R., et al., 2005a, Phys. Rev. Lett. 95, 182302.

Arnold, R., et al., 2005b, Nucl. Instrum. Methods Phys. Res. A 536, 79.

Ashie, Y., et al., 2004, Phys. Rev. Lett. 93, 101801.

Ashie, Y., et al., 2005, Phys. Rev. D 71, 112005.

Audi, G., A. H. Wapstra, and C. Thibault, 2003, Nucl. Phys. A 729, 337.

Aunola, M., and J. Suhonen, 1996, Nucl. Phys. A 602, 133.

Avignone, F. T., III, and R. L. Brodzinski, 1988, Prog. Part. Nucl. Phys. 21, 99.

Avignone, F. T., III, G. S. King III, and Y. Zdesenko, 2005, New J. Phys. 7, 6.

Avignone, F. T., III, et al., 1992, Nucl. Phys. B (Proc. Suppl.) 28A, 280.

Bahcall, J., H. Murayama, and C. Peña-Garay, 2004a, Phys. Rev. D 70, 033012.

Bahcall, J. N., H. Murayama, and C. Peña-Garay, 2004b, Phys. Rev. D 70, 033012.

Barabash, A., 2006a, Czech. J. Phys. 56, 437.

Barabash, A. S., (NEMO), 2004, Phys. At. Nucl. 67, 1984.

Barabash, A. S., 2005, Phys. At. Nucl. 68, 414.

Barabash, A. S., (NEMO), 2006b, e-print arXiv:hep-ex/ 0610025 .

Bardelli, L., et al., 2006, Nucl. Instrum. Methods Phys. Res. A 569, 743.

Barger, V., S. L. Glashow, P. Langacker, and D. Marfatia, 2002, Phys. Lett. B 540, 247.
Baudis, L., et al., (Heidelberg-Moscow), 1999, Phys. Rev. Lett. 83, 41.

Bazarko, A., (MiniBooNE Collaboration) 2000, Nucl. Phys. B, Proc. Suppl. 91, 210.

Beier, E., T. Onstott, R. Robertson, B. Sadoulet, and J. Tiedje, 2006, Deep Science-the Report of the S1 Principal Investigators on the Science and Engineering Case for a Deep Underground Science and Engineering Laboratory (Publications Office, Fermilab, Chicago).

Bellini, G., et al., 1996, Nucl. Phys. B (Proc. Suppl.) 48, 363.

Bellini, G., et al., 2001, Eur. Phys. J. C 19, 43.

Bender, M., P.-H. Heenen, and P.-G. Reinhard, 2003, Rev. Mod. Phys. 75, 121.

Bernabei, R., et al., 2002, Phys. Lett. B 546, 23.

Bernatowicz, T., et al., 1993, Phys. Rev. C 47, 806.

Bhattacharyya, G., H. V. Klapdor-Kleingrothaus, H. Päs, and A. Pilaftsis, 2003, Phys. Rev. D 67, 113001.

Bilenky, S. M., A. Faessler, and F. Šimkovic, 2004, Phys. Rev. D 70, 033003.

Bilenky, S. M., and S. T. Petcov, 1987, Rev. Mod. Phys. 59, 671. Bobyk, A., W. A. Kaminski, and F. Šimkovic, 2001, Phys. Rev. C 63, 051301(R).

Boger, J., et al., 2000, Nucl. Instrum. Methods Phys. Res. A 449, 172.

Bogner, S. K., T. T. S. Kuo, and A. Schwenk, 2003, Phys. Rep. 386, 1.

Brodzinski, R. L., H. S. Miley, J. H. Reeves, and F. T. Avignone, III, 1990, Nucl. Instrum. Methods Phys. Res. A 292, 337.

Buchmüller, W., P. D. Bari, and M. Plümacher, 2002, Phys. Lett. B 547, 128.

Caccianiga, B., and M. G. Giammarchi, 2001, Astropart. Phys. 14, 15 .

Caurier, E., G. Martnez-Pinedo, F. Nowacki, A. Poves, and A. P. Zuker, 2005, Rev. Mod. Phys. 77, 427.

Caurier, E., J. Menendez, F. Nowacki, and A. Poves, 2007, e-print arXiv:0709.2137.

Caurier, E., F. Nowacki, and A. Poves, 2007, e-print arXiv:0709.0277.

Caurier, E., F. Nowacki, A. Poves, and J. Retamosa, 1996, Phys. Rev. Lett. 77, 1954.

Chen, M., 2005, Nucl. Phys. B, Proc. Suppl. 145, 65.

Cheoun, M. K., A. Bobyk, A. Faessler, F. Šimkovic, and G. Teneva, 1993, Nucl. Phys. A 561, 74.

Chikashige, Y., R. N. Mohapatra, and R. D. Peccei, 1981, Phys. Lett. 98B, 265.

Chikira, Y., N. Haba, and Mimura Y., 2000, Eur. Phys. J. C 16, 701.

Chun, E. J., C. W. Kim, and U. W. Lee, 1998, Phys. Rev. D 58, 093003.

Cirigliano, V., A. Kurylov, M. J. Ramsey-Musolf, and P. Vogel, 2004, Phys. Rev. Lett. 93, 231802.

Civitarese, O., and J. Suhonen, 2003a, Nucl. Phys. A 729, 867. Civitarese, O., and J. Suhonen, 2003b, Nucl. Phys. A 761, 313. Cowell, S., 2006, Phys. Rev. C 73, 028501.

Danevich, F. A., 2001, Nucl. Phys. A 694, 375.

Danevich, F. A., et al., 2000, Phys. Rev. C 62, 045501.

Danevich, F. A., et al., 2003, Phys. Rev. C 68, 035501.

Danevich, F. A., et al., 2005a, Nucl. Instrum. Methods Phys. Res. A 541, 583.

Danevich, F. A., et al., 2005b, Nucl. Instrum. Methods Phys. Res. A 544, 553.

Danevich, F. A., et al., 2006, Nucl. Instrum. Methods Phys. Res. 
A 556, 259.

Danilov, M., et al., 2000, Phys. Lett. B 480, 12.

Davis, R., Jr., 1955, Phys. Rev. 97, 766.

Deppisch, F., and H. Päs, 2007, Phys. Rev. Lett. 98, 232501.

Di Marco, M., et al., 2007, Nucl. Phys. B, Proc. Suppl. 172, 45.

Doi, M., T. Kotani, and E. Takasugi, 1985, Prog. Theor. Phys. 83, 1 .

Ejiri, H., 2000, Phys. Rep. 338, 265.

Ejiri, H., 2005, J. Phys. Soc. Jpn. 74, 2101.

Ejiri, H., 2006a, Czech. J. Phys. 56, 459.

Ejiri, H., 2006b, Prog. Part. Nucl. Phys. 57, 153.

Ejiri, H., et al., 1991a, Nucl. Instrum. Methods Phys. Res. A 302, 304.

Ejiri, H., et al., 1991b, Phys. Lett. B 258, 17.

Ejiri, H., et al., 2000, Phys. Rev. Lett. 85, 2917.

Ejiri, H., et al., 2001, Phys. Rev. C 63, 065501.

Elgaroy, O., and O. Lahov, 2003, J. Cosmol. Astropart. Phys. 04, 004.

Elliott, S., V. Gehman, K. Kazkaz, D.-M. Mei, and A. Young, 2006, Nucl. Instrum. Methods Phys. Res. A 588, 504.

Elliott, S. R., 2003, Int. J. Mod. Phys. A 18, 4097.

Elliott, S. R., 2006, e-print arXiv:nucl-ex/0609024.

Elliott, S. R., and J. Engel, 2004, J. Phys. G 30, R183.

Elliott, S. R., A. Hahn, and M. K. Moe, 1987, Phys. Rev. Lett. 59, 2020.

Elliott, S. R., and P. Vogel, 2002, Annu. Rev. Nucl. Part. Sci. 52, 115.

Engel, J., M. Bender, J. Dobaczewski, W. Nazarewicz, and R. Surman, 1999, Phys. Rev. C 60, 014302.

Engel, J., and P. Vogel, 2004, Phys. Rev. C 69, 034304.

Engel, J., P. Vogel, and M. R. Zirnbauer, 1988, Phys. Rev. C 37, 731.

Faessler, A., and F. Šimkovic, 1998, J. Phys. G 24, 2139.

Feruglio, F., A. Strumia, and F. Vissani, 2002, Nucl. Phys. B 637, 345.

Fetter, J., G. C. McLaughlin, A. B. Balantekin, and G. M. Fuller, 2003, Astropart. Phys. 18, 433.

Fiorini, E., and T. Niinikoski, 1984, Nucl. Instrum. Methods Phys. Res. A 224, 83.

Fireman, E., 1949, Phys. Rev. 75, 323.

Fireman, E., 1952, Phys. Rev. 86, 451.

Fogli, G. L., E. Lisi, A. Marrone, and A. Palazzo, 2006, Prog. Part. Nucl. Phys. 57, 742.

Freeman, S. J., et al., 2007, Phys. Rev. C 75, 051301.

Fukugita, M., and T. Yanagida, 1986, Phys. Lett. B 174, 45.

Furry, W. H., 1939, Phys. Rev. 56, 1184.

Gaitskell, R., et al., (MAJORANA), 2003, e-print arXiv:nuclex/0311013.

Gehman, V. M., and S. R. Elliott, 2007, J. Phys. G 34, 667.

Gell-Mann, M., et al., 1979, Supergravity (North-Holland Amsterdam), p. 315.

Gelmini, G. B., and M. Roncadelli, 1981, Phys. Lett. 99B, 411.

Georgi, H. M., S. L. Glashow, and S. Nussinov, 1981, Nucl. Phys. B 193, 297.

Goeppert-Mayer, M., 1935, Phys. Rev. 48, 512.

Goldman, T., G. J. Stephenson, Jr., and B. H. J. McKellar, 2000, Mod. Phys. Lett. A 15, 439.

Gößling, C., M. Junker, H. Kiel, D. Muenstermann, S. Oehl, and K. Zuber, 2005, Phys. Rev. C 72, 064328.

Goswami, S., and W. Rodejohann, 2006, Phys. Rev. D 73, 113003.

Hableib, J. A., and R. A. Sorensen, 1967, Nucl. Phys. A 98, 542 .
Hagino, K., N. V. Giai, and H. Sagawa, 2004, Nucl. Phys. A 731, 264.

Haller, E. E., et al., 1982, Proceedings of the 4th International Conference on Neutron Transmutation Doping of Semiconductor Materials, Gaithersburg, MD (Larabee Plenum Press, New York), p. 21.

Hannested, S., 2003, Proceedings of the International Conference: Thinking, Observing, and Mining the Universe (World Scientific, Singapore)

Hara, K., 1964, Prog. Theor. Phys. 32, 88.

Harney, H. L., 2001, Mod. Phys. Lett. A 16, 2409.

Haxton, W. C., and G. J. Stephenson, Jr., 1984, Prog. Part. Nucl. Phys. 12, 409.

Heusser, G., 1995, Annu. Rev. Nucl. Part. Sci. 45, 543.

Hirsch, J. G., O. Castanos, and P. O. Hess, 1995, Nucl. Phys. A 582, 124.

Hirsch, M., H. Klapdor-Kleingrothaus, and S. Kovalenko, 1996a, Phys. Lett. B 374, 7.

Hirsch, M., H. Klapdor-Kleingrothaus, and S. Kovalenko, 1996b, Phys. Lett. B 372, 181.

Hirsch, M., H. Klapdor-Kleingrothaus, and S. Kovalenko, 1996c, Phys. Rev. D 54, R4207.

Hjorth-Jensen, M., T. T. S. Kuo, and E. Osnes, 1995, Phys. Rep. 261, 125.

Horoi, M., S. Stoica, and B. Brown, 2007, Phys. Rev. C 75, 034303.

Ingram, M. G., and J. H. Reynolds, 1950, Phys. Rev. 78, 822.

Ishihara, N., 2007, private communication.

Ishihara, N., et al., 2000, Nucl. Instrum. Methods Phys. Res. A 443, 101.

Kaplan, D., A. Nelson, and N. Weiner, 2004, Phys. Rev. Lett. 93, 091801.

Klapdor-Kleingrothaus, H., and U. Sarkar, 2003, Phys. Lett. B 554, 45.

Klapdor-Kleingrothaus, H., et al., 2001a, Eur. Phys. J. A 12, 147.

Klapdor-Kleingrothaus, H. V., 1997, Workshop on Physics Beyond the Desert: Accelerator and Non-Accelerator Approaches (IOP, Bristol).

Klapdor-Kleingrothaus, H. V., O. Chkvorez, I. V. Krivosheina, and C. Tomei, 2003, Nucl. Instrum. Methods Phys. Res. A 511, 355.

Klapdor-Kleingrothaus, H. V., A. Deitz, I. V. Krivosheina, and O. Chkvorez, 2004a, Nucl. Instrum. Methods Phys. Res. A 522, 371.

Klapdor-Kleingrothaus, H. V., A. Deitz, I. V. Krivosheina, and O. Chkvorez, 2004b, Phys. Lett. B 586, 198.

Klapdor-Kleingrothaus, H. V., and I. V. Krivosheina, 2006, Mod. Phys. Lett. A 21, 1547.

Klapdor-Kleingrothaus, H. V., et al., 2001b, Workshop on Neutrino Oscillations and their Origins (World Scientific, Singapore).

Klapdor-Kleingrothaus, H. V., et al., 2003, Nucl. Instrum. Methods Phys. Res. A 511, 341.

Kobzarev, I. Y., et al., 1980, Sov. J. Nucl. Phys. 32, 823.

Kortelainen, M., O. Civitarese, J. Suhonen, and J. Toivanen, 2007, Phys. Lett. B 647, 128.

Kortelainen, M., and J. Suhonen, 2002, Europhys. Lett. 58, 666.

Kortelainen, M., and J. Suhonen, 2007, Phys. Rev. C 75, 051303(R).

Kowalski, K., D. Dean, M. Hjorth-Jensen, T. Papenbrock, and P. Piecuch, 2004, Phys. Rev. Lett. 92, 132501. 
Kraus, C., et al., 2005, Eur. Phys. J. C 40, 447.

Lobashev, V. M., et al., 1999, Phys. Lett. B 460, 227.

Majorana, E., 1937, Nuovo Cimento 14, 171.

Maltoni, M., and T. Schwetz, 2007, e-print arXiv:0705.0107.

Mei, D.-M., S. R. Elliott, V. M. Gehman, A. Hime, and K.

Kazkaz, 2007, e-print arXiv:0704.0306v2.

Mei, D.-M., and A. Hime, 2006, Phys. Rev. D 73, 053004.

Miller, G. A., and J. E. Spencer, 1976, Ann. Phys. (N.Y.) 100, 562.

Moe, M., and P. Vogel, 1994, Annu. Rev. Nucl. Part. Sci. 44, 247.

Moe, M. K., 1991, Phys. Rev. C 44, R931.

Mohapatra, R., and G. Senjanovic, 1980, Phys. Rev. Lett. 44, 912.

Mohapatra, R. N., 1999, Nucl. Phys. B (Proc. Suppl.) 77, 376.

Nakamura, H., et al., 2006, J. Phys.: Conf. Ser. 39, 350.

Nakamura, H., et al., 2007, J. Phys. Soc. Jpn. 76, 11420.

Navratil, P., J. P. Vary, and B. R. Barrett, 2000, Phys. Rev. C 62, 054311.

Nygren, D., 2007, private communication.

Ogawa, I., et al., 2004, Nucl. Phys. A 730, 215.

Pandola, L., et al., 2007, Nucl. Instrum. Methods Phys. Res. A 570, 149.

Pantis, G., F. Šimkovic, J. Verdagos, and A. Faessler, 1996, Phys. Rev. C 53, 695.

Pantis, G., and J. Vergados, 1990, Phys. Lett. B 242, 1.

Papenbrock, T., and D. J. Dean, 2003, Phys. Rev. C 67, 051303.

Pascoli, S., and S. Petcov, 2003, e-print arXiv:hep-ph/0308034.

Pieper, S. C., and R. B. Wiringa, 2001, Annu. Rev. Nucl. Part. Sci. 51, 53.

Piepke, A., 2007, private communication.

Poves, A., E. Caurier, and F. Nowacki, 2007, e-print arXiv:0709.0277.

Prézeau, G., 2006, Phys. Lett. B 633, 93.

Prézeau, G., M. Ramsey-Musolf, and P. Vogel, 2003, Phys. Rev. D 68, 034016.

Primakoff, H., 1952, Phys. Rev. 85, 888.

Primakoff, H., and S. P. Rosen, 1981, Annu. Rev. Nucl. Part. Sci. 31, 145.

Racah, G., 1937, Nuovo Cimento 14, 322.

Raduta, A. A., A. Faessler, and S. Stoica, 1991, Nucl. Phys. A 534, 149.

Redshaw, M., et al., 2007, Phys. Rev. Lett. 98, 053003.

Rodin, V., and A. Faessler, 2006, Prog. Part. Nucl. Phys. 57, 226.

Rodin, V., A. Faessler, F. Šimkovic, and P. Vogel, 2003, Phys. Rev. C 68, 044302.

Rodin, V. A., A. Faessler, F. Šimkovic, and P. Vogel, 2006, Nucl. Phys. A 766, 107; erratum arXiv:0706.4304, Nucl. Phys. A (to be published).

Roth, R., H. Hergert, P. Papakonstantinou, T. Neff, and H. Feldmeier, 2005, Phys. Rev. C 72, 034002.

Rowe, D. J., 1968, Rev. Mod. Phys. 40, 153.

Schechter, J., F. Šimkovic, and A. Faessler, 1996, Nucl. Phys. A 600, 179.

Schechter, J., and J. W. F. Valle, 1982, Phys. Rev. D 25, 2951. Schönert, S., et al., 2005, Nucl. Phys. B, Proc. Suppl. 145, 242. Siiskonen, T., M. H.-J. T., and J. Suhonen, 2001, Phys. Rev. C 63, 055501.
Šimkovic, F., and A. Faessler, 2002, Prog. Part. Nucl. Phys. 48, 201.

Šimkovic, F., A. Faessler, V. Rodin, P. Vogel, and J. Engel, 2007, e-print arXiv:0710.2055.

Šimkovic, F., L. Pacearescu, and A. Faessler, 2003, Nucl. Phys. A 733, 321.

Šimkovic, F., G. Pantis, J. D. Vergados, and A. Faessler, 1999, Phys. Rev. C 60, 055502.

Šimkovic, F., S. Schwinger, M. Veselski, G. Pantis, and A. Faessler, 1997, Phys. Lett. B 393, 267.

Šimkovic, F., et al., 2001, J. Phys. G 27, 2233.

Sorel, M., J. M. Conrad, and M. H. Shaevitz, 2004, Phys. Rev. D 70, 073004.

Stephenson, G. J., Jr., T. Goldman, B. H. J. McKellar, and M. Garbutt, 2005, Int. J. Mod. Phys. A 20, 6373.

Stoica, S., and H. V. Klapdor-Kleingrothaus, 2001, Nucl. Phys. A 694, 269.

Sugiyama, H., 2004, The 4th Workshop on Neutrino Oscillations and their Origins, NOON 2003 (World Scientific, Singapore).

Suhonen, J., 2005, Phys. Lett. B 607, 87.

Suhonen, J., and O. Civitarese, 1998, Phys. Rep. 300, 124.

Suhonen, J., P. C. Divari, L. D. Skouras, and I. P. Johnstone, 1997, Phys. Rev. C 55, 714.

Sujkowski, Z., and S. Wycech, 2004, Phys. Rev. C 70, 052501.

Takeuchi, Y., 2008, High Energy Physics-ICHEP 04, Proceedings of the 32nd International Conference (World Scientific, Singapore).

Toivanen, J., and J. Suhonen, 1995, Phys. Rev. Lett. 75, 410.

Tomoda, T., 1991, Rep. Prog. Phys. 54, 53.

Tomoda, T., 2000, Phys. Lett. B 474, 245.

Tretyak, V., and Yu G. Zdesenko, 2002, At. Data Nucl. Data Tables 80, 83.

Turkevich, A. L., T. E. Economou, and G. A. Cowan, 1991, Phys. Rev. Lett. 67, 3211.

Umehara, S., et al., 2006, J. Phys.: Conf. Ser. 39, 356.

Vergados, J., 2002, Phys. Rep. 361, 1.

Vogel, P., and M. R. Zirnbauer, 1986, Phys. Rev. Lett. 57, 3148. Volpe, C., 2005, J. Phys. G 31, 903.

Wang, S. C., H. T. Wong, and M. Fujiwara, 2002, Nucl. Instrum. Methods Phys. Res. A 479, 498.

Weinberg, S., 1979, Phys. Rev. Lett. 43, 1566.

White, G. K., S. J. Collocott, and G. J. Collins, 1990, J. Phys.: Condens. Matter 2, 7715.

Wloch, M., D. J. Dean, J. R. Gour, M. Hjorth-Jensen, K. Kowalski, T. Papenbrock, and P. Piecuch, 2005, Phys. Rev. Lett. 94, 212501.

Yanagida, T., 1979, Proceedings of the Workshop on Unified Theory and Baryon Number in the Universe (KEK, Tsuuba, Japan).

Yao, W.-M., et al., (Particle Data Group), 2006, J. Phys. G 33, 1. Zdesenko, Yu. G., 2002, Rev. Mod. Phys. 74, 663.

Zdesenko, Yu. G., F. A. Danevich, and V. I. Tretyak, 2002, Phys. Lett. B 546, 206.

Zdesenko, Yu. G., O. A. Pnnkratenko, and V. I. Tretyak, 2001, J. Phys. G 27, 2129.

Zdesenko, Yu. G., et al., 2005, Astropart. Phys. 23, 249.

Zuber, K., 2001, Phys. Lett. B 519, 1.

Zuber, K., 2005, e-print arXiv:nucl-ex/0511009. 